DOT/FAA/AM-97/25

Office of Aviation Medicine

Washington, DC 20591

\title{
Workshift and Antihistamine Effects on Task Reffomance
}

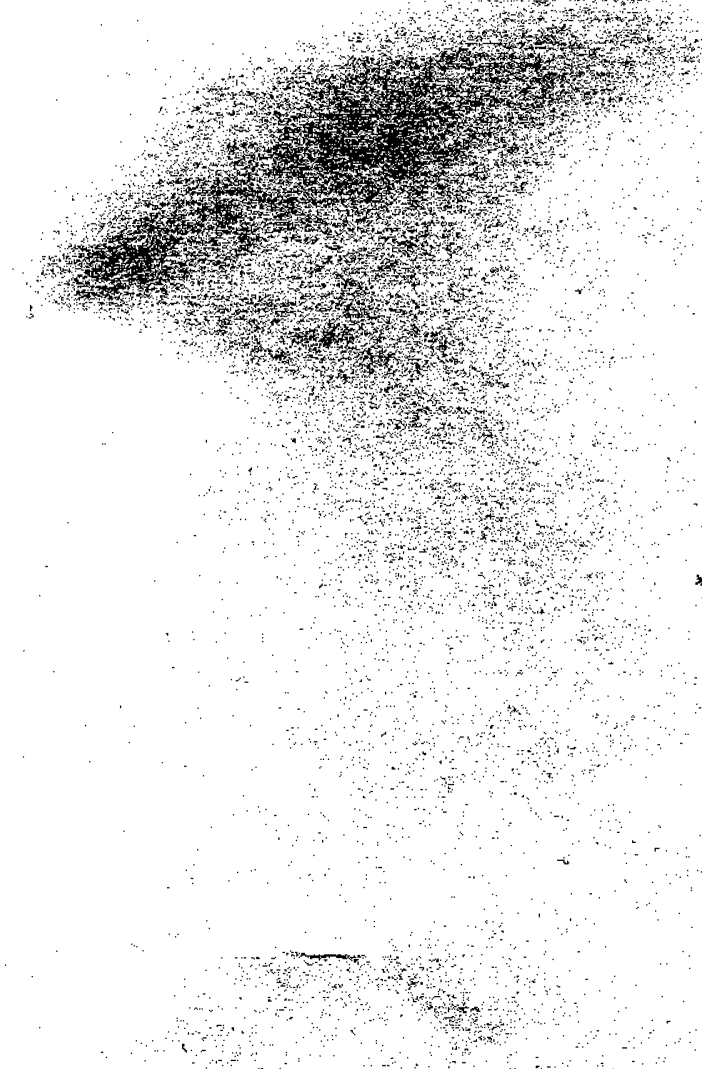

Thomas E Nesthus

Civil Aeromedical Institute

Federal Aviation Administration

Oklahoma City, OK 73125

December 1997

Final Report
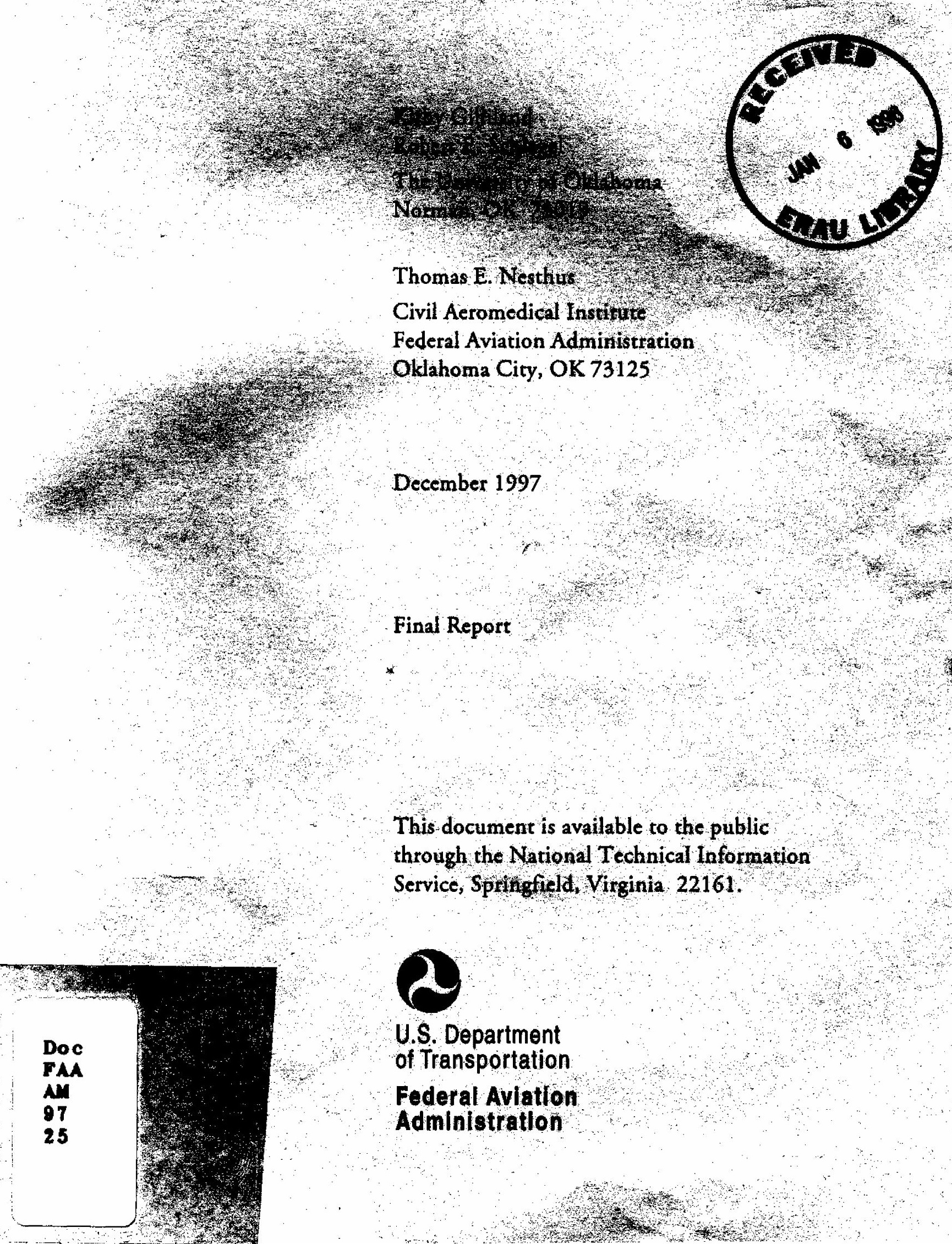

This document is available to the public through the National Technical Information Service, Springfield, Virginia 22161.
U.S. Department
of Transportation
Federal Aviation Administration 


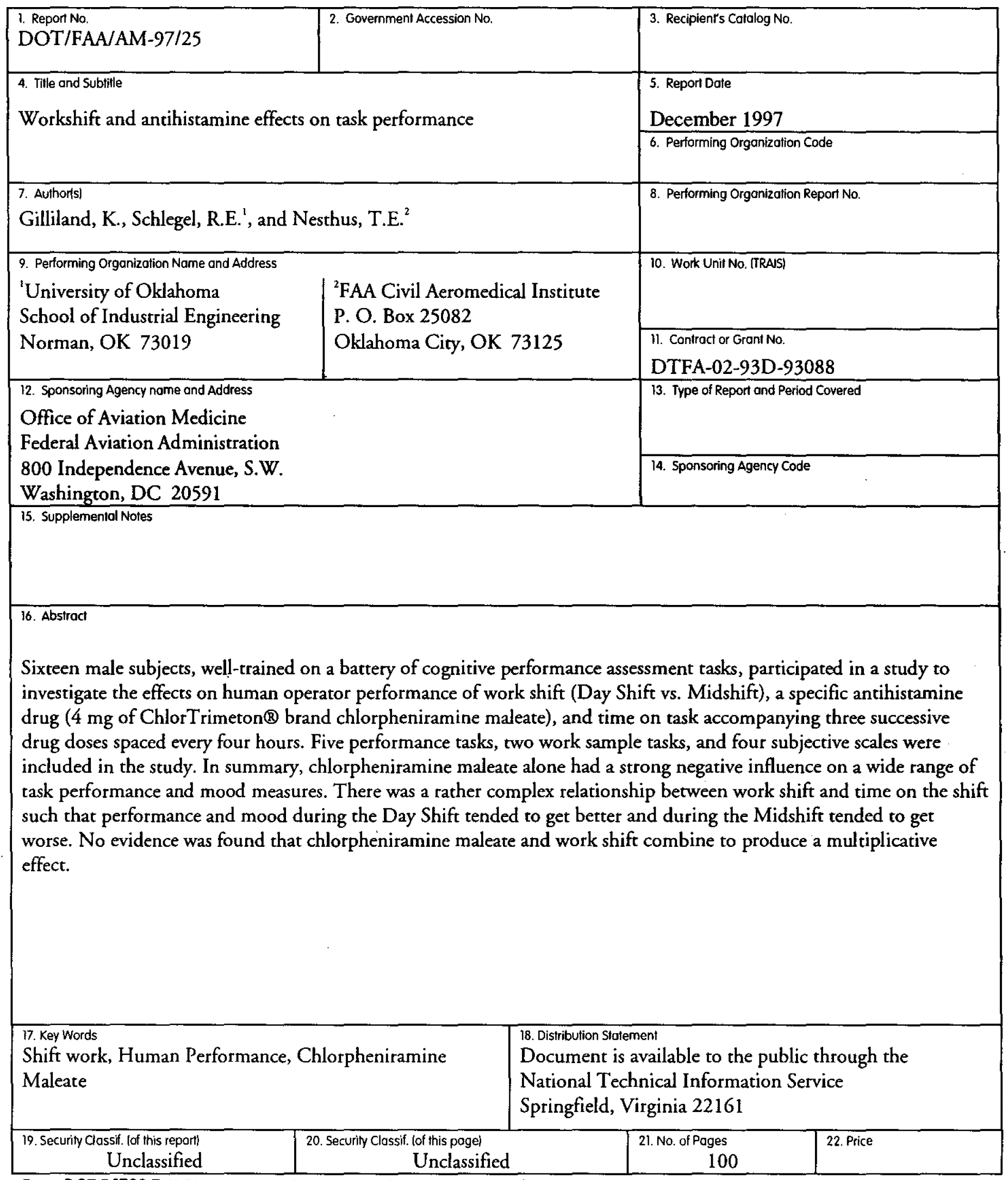

Form DOT F 1700.7 (8-72) Reproduction of completed page authorized 


\section{PREFACE}

This is a report of a Civil Aeromedical Institute (CAMI) project completed at the University of Oklahoma under Task Order DTFA-02-94-T80067 of contract DTFA-02-93-D-93088 for the Federal Aviation Administration. Funding for the effort was provided by the CAMI Human Factors Research Laboratory (CAMI/AAM-500).

The authors gratefully acknowledge several individuals for their contributions to the project. Luz-Eugenia Cox-Fuenzalida, Ioannis Vasmatzidis, Rhonda Swickert, and Randa L. Shehab served to coordinate the numerous facets of the study. Their contributions to the recruitment and retention of subjects, and to the collection, reduction, and analysis of data were invaluable. Scott Mills provided valuable hardware and software support and Arasendran Sellakannu and Tamy L. Fry made important contributions to the collection, reduction, summarization, and analysis of the data. The graduate and undergraduate assistants who worked on this project devoted long hours, often at unusual times of the day, to the collection of the data reported here. We appreciate their efforts very much.

The authors are also very grateful to Richard D. Havel, M.D., Director of Goddard Health Center at the University of Oklahoma, who served as the Medical Monitor for this project. Dr. Havel generously provided his time, interest, and support for this project.

Finally, the authors thank Dr. Robert E. Blanchard and Dr. David J. Schroeder of CAMI for their general guidance and their comments on drafts of this report. 


\section{TABLE OF CONTENTS}

1.0 INTRODUCTION Page

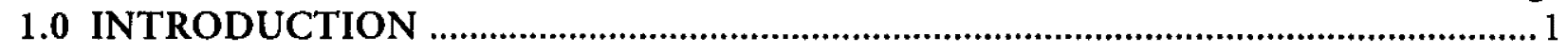

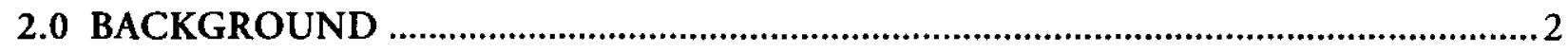

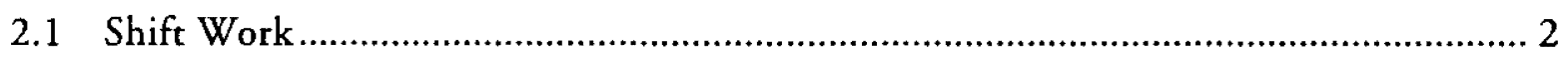

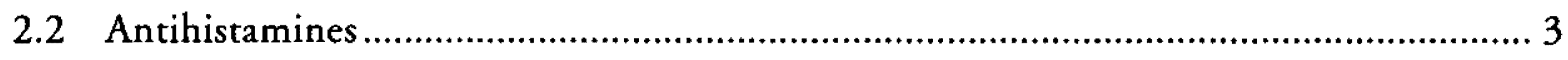

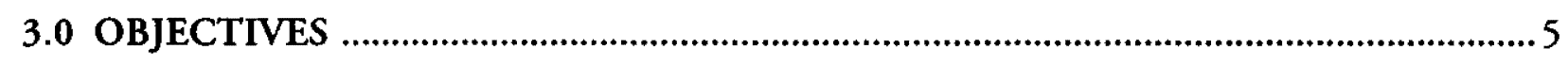

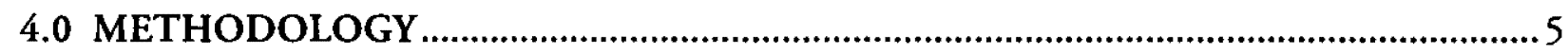

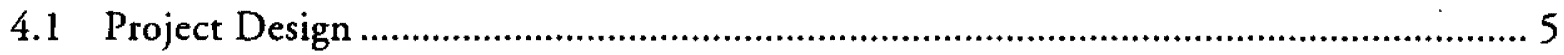

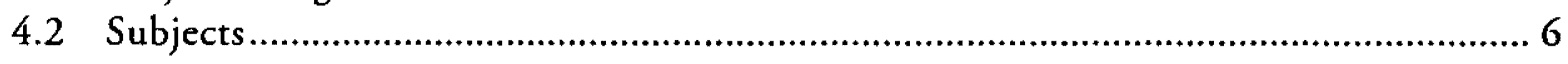

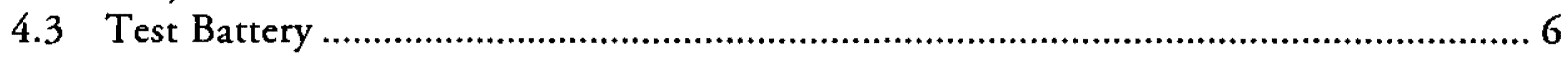

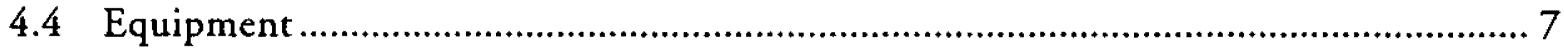

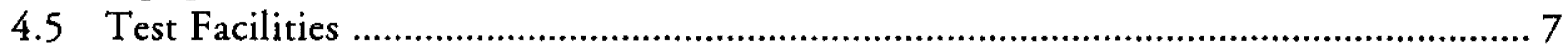

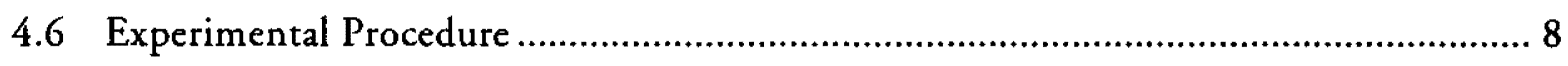

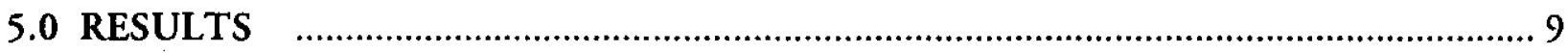

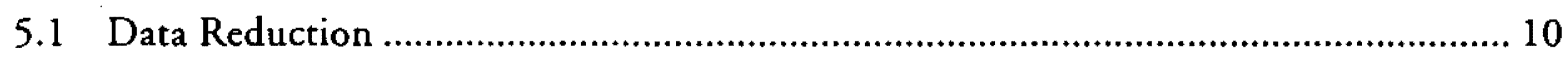

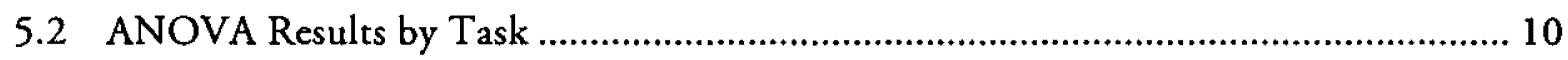

5.3 Subjective (Self-Report) Measures .................................................................. 14

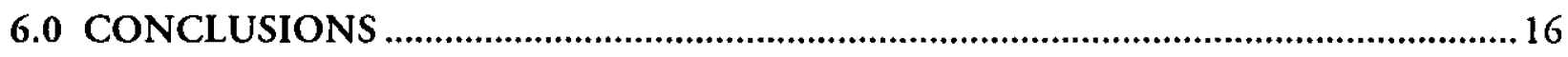

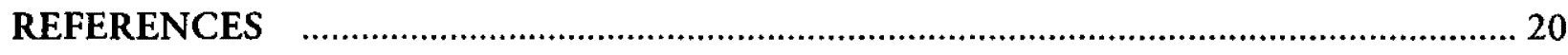

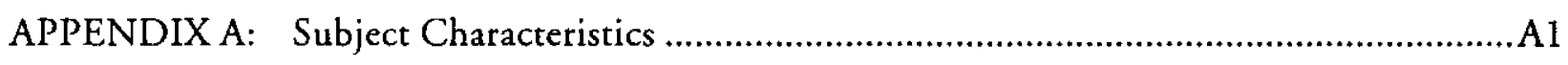

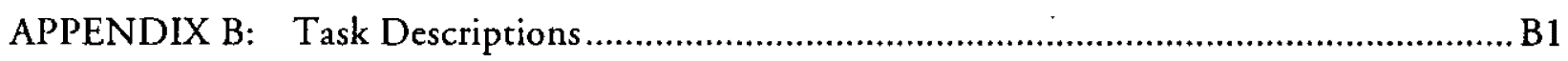

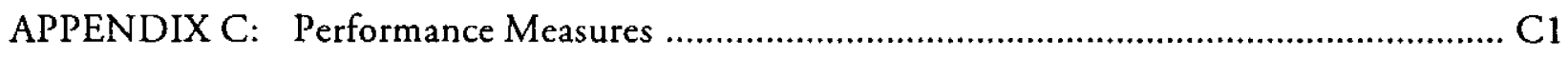




\section{LIST OF FIGURES}

Figure

1. Laboratory Model of Readiness-to-Perform Testing …........................................... 25

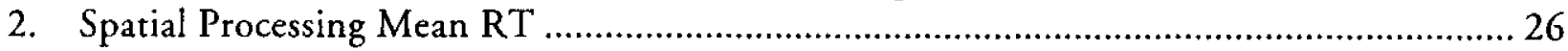

3. Spatial Processing Percent Correct ............................................................................... 27

4. Critical Tracking Mean Lambda ................................................................................ 28

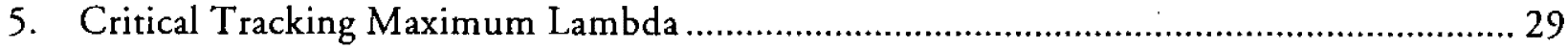

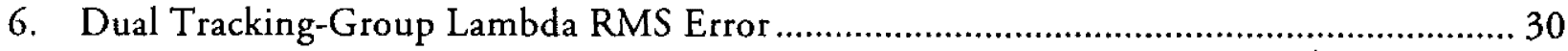

7. Dual Tracking-Group Lambda Control Losses ....................................................... 31

8. Dual Memory Search-Group Lambda Mean RT ..................................................... 32

9. Dual Memory Search-Group Lambda Percent Correct ................................................. 33

10. Dual Memory Search-Group Lambda Throughput ..................................................... 34

11. Dual Tracking-Individual Lambda RMS Error ........................................................... 35

12. Dual Tracking-Individual Lambda Control Losses ........................................................ 36

13. Dual Memory Search-Individual Lambda Mean RT .................................................... 37

14. Dual Memory Search-Individual Lambda Percent Correct ............................................. 38

15. Dual Memory Search-Individual Lambda Throughput ................................................. 39

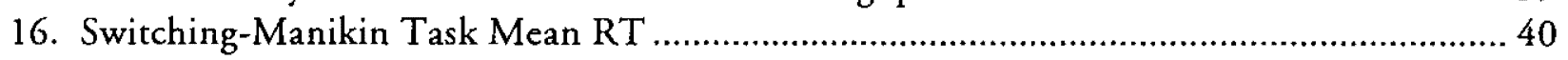

17. Switching-Manikin Task Mean RT for Transitions ................................................... 41

18. Switching-Manikin Task Percent Correct .................................................................. 42

19. Switching-Manikin Task Percent Correct for Transitions ............................................. 43

20. Switching-Manikin Task Throughput .................................................................. 44

21. Switching-Mathematical Processing Mean RT ...................................................... 45

22. Switching-Mathematical Processing Mean RT for Transitions ...................................... 46

23. Switching-Mathematical Processing Percent Correct ................................................. 47

24. Switching-Mathematical Processing Percent Correct for Transitions ............................. 48

25. Switching-Mathematical Processing Throughput ..................................................... 49

26. NovaScan TM_Visual Search and Vector Projection Task Mean RT ................................ 50

27. NovaScanTM_Visual Search and Vector Projection Percent Correct ................................. 51

28. NovaScan ${ }^{\mathrm{TM}}$ _Continuous Spatial Memory Task Mean RT ............................................ 52

29. NovaScan ${ }^{\mathrm{TM}}$-Continuous Spatial Memory Task Percent Correct .................................... 53

30. NovaScan ${ }^{\mathrm{TM}}$-Attention Task Percent Correct ................................................................ 54

31. Air Traffic Scenarios Test Percent at Destination ........................................................... 55

32. Air Traffic Scenarios Test Delay .............................................................................. 56

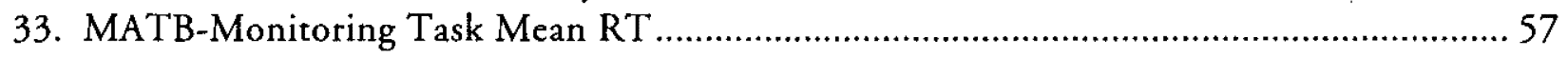

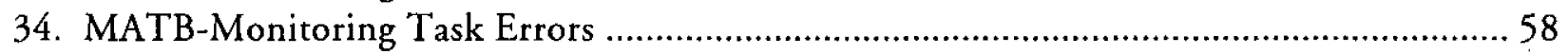

35. MATB-Communications Task Mean RT …............................................................. 59

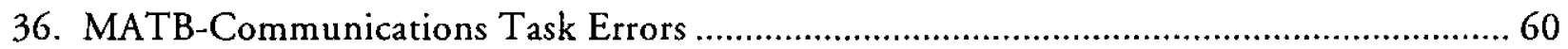

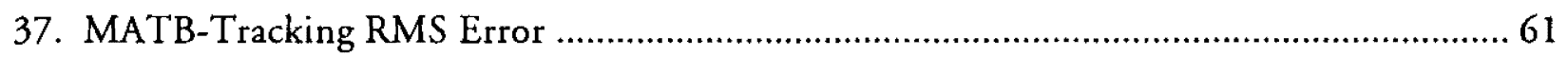

38. MATB-Resource Management Task Mean Absolute Deviation ......................................62

39. Antihistamine Symptoms Questionnaire Mean Score ..................................................6. 63 
Figure

40. Activity State Questionnaire Mean PHYSICAL Score ...............................................6 64

41. Activity State Questionnaire Mean PREP Score .........................................................65

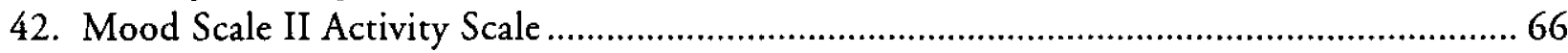

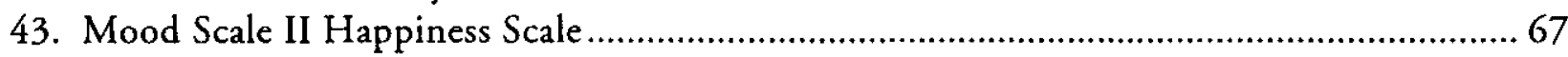

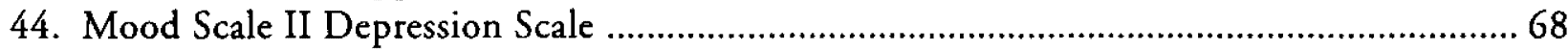

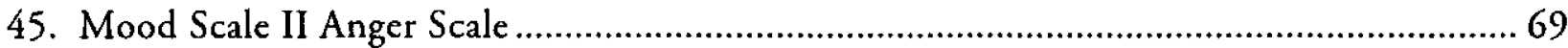

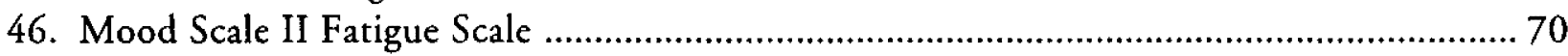

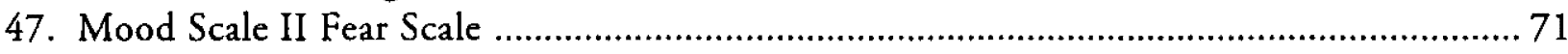

48. Mood Scale II Response Time ....................................................................................... 72

\section{LIST OF TABLES}

Table

1. Summary of Task Codes

2. Example Protocols for Dosing and Testing during Day Shift and Midshift Sessions

3. Individualized Lambda Values for Dual Task

4. Summary of Significant ANOVA Effects 


\section{EXECUTIVE SUMMARY}

Considerable concern has been raised in recent years about factors in the work place that compromise the effectiveness of workers. Of special concern for the aviation environment is the fact that shift work appears to have negative effects on sleep patterns, selfreported satisfaction with working conditions, and task performance. In 1983, Schroeder and Goulden compiled a bibliography of research related to shift work. At that time, over 1300 studies had been published - a clear indication of the level of concern for this issue. Such concern seems all the more relevant today, especially for Air Traffic Control Specialists (ATCSs) who have in the U.S. historically worked a rapid rotating shift schedule called the "2-2-1". This schedule involves working two afternoon shifts, followed by two morning shifts, and one "midshift" or night shift, within a five-day period. Recent laboratory and field research suggests that such schedules do disrupt sleep patterns among ATCSs. Further evidence based on a wide range of jobs in the aviation environment supports this finding.

Also of increasing concern for the aviation environment is a recent finding regarding common over-thecounter (OTC) drug use. A comprehensive survey of aviation accidents involving fatalities revealed that OTC antihistamines were among the most frequently found drugs. While antihistamines were present in only $3.7 \%$ of the 1845 fatalities with positive toxicology results between 1989 and 1993, they presented a relatively high frequency rate compared to all other drugs. As early as 1968, Federal Aviation Administration (FAA) researchers had discovered that the administration of one common antihistamine, chlorpheniramine maleate, significantly reduced psychomotor performance. Equally important was the additional finding that, while both chlorpheniramine maleate and altitude had significant negative effects on psychomotor performance, there was a negative "synergistic effect" such that performance decrements under the two factors in combination were much greater than the simple sum of the decrements for each factor.
Such findings raise serious concerns regarding the effects of shift work and OTC antihistamine use in the aviation environment. Perhaps more troubling is the possibility that antihistamines may well be used during shift work, thereby possibly compounding these negative effects. The purpose of the study reported here was to investigate the effects of work shift (Day Shift vs. Midshift) and a specific antihistamine drug (chlorpheniramine maleate) on human operator performance. In addition, the effect of time on task, or successive drug doses, through the shift (Session effect) was also assessed. A battery of cognitive and human performance tasks was used to investigate these effects, as well as various self-report measures of physical state, mood, and workload.

Sixteen male subjects, well-trained on the battery of tasks, participated in the study. Subjects ranged in age from 21 to 38 years with a mean of 25.9 and a standard deviation of 5.6 years. All subjects were surveyed for self-reported normal (or corrected-tonormal) vision, normal hearing, and the absence of any central nervous system stimulant or depressant medications. Due to the nature of the study, additional relevant information about alcohol, caffeine, medication, and possible drug use was obtained. On average, subjects consumed 3.8 alcoholic beverages per week. Average coffee consumption was 2.6 cups per day, and average cola consumption was 1.5 cans per day.

Several factors were considered in selecting tasks for this study. One critical factor was the specific information processing skills necessary in typical safetysensitive jobs, such as air traffic control or aircraft piloting. Another relevant issue was the information provided by a specific task with respect to the cognitive processes or information processing stages affected by one or more risk factors. These and other factors were taken into account in reviewing a large number of human performance task batteries. As a result, five performance tasks (Spatial Processing, Critical Tracking, Dual Task-Tracking / Sternberg Memory, Attention Switching-Manikin Task / 
Mathematical Processing, and the NovaScan TM FAA Task), two work sample tasks (Air Traffic Scenarios Test and Multi-Attribute Task Battery), and four subjective scales (Antihistamine Symptoms Questionnaire, Activity State Questionnaire, Mood Scale II, and NASA Task Load Index) were selected for inclusion in the study.

All tasks were presented on eight microcomputer workstations. Each workstation consisted of a Gateway $486-33 \mathrm{MHz}$ processor with the necessary in put devices ("Anykey" keyboard, Microsoft mouse, Kensington Expert Mouse 4.0 trackball, $\mathrm{CH}$ Products Flightstick, and NovaScan ${ }^{\mathrm{TM}}$ interface box). All data were recorded on these machines and on subject diskettes and then downloaded to a central data management system. The software automatically performed all functions such as subject identification, file naming, test sequencing, and data backup.

Testing was conducted on four weekends with all subjects tested under all four of the drug-shift combinations. Two of the weekends involved "Day Shift" testing and two weekends involved "Midshift" testing. Day Shift testing began on Saturday mornings at 8:00 a.m. or 10:00 a.m. and ended 11 hours later. Midshift testing began on Friday evenings at 8:00 p.m. or 10:00 p.m. and also lasted 11 hours. Day Shift and Midshift testing conditions were randomly assigned to the four weekend test days. Subjects were randomly assigned to counterbalanced orderings of the antihistamine-placebo conditions on both Day Shift and Midshift test days.

Subjects were advised to eat only light meals well in advance of the initial test session and to avoid any foods that might slow drug absorption rate (e.g., high fat foods or dairy products). On each test day, subjects entered the laboratory, completed some general questionnaires, and were given a beverage containing approximately $6 \mathrm{oz}$ of fruit juice, $1 \mathrm{oz}$ of crushed ice, and either $4 \mathrm{mg}$ of ChlorTrimeton ${ }^{\circledR}$ brand chlorpheniramine maleate in syrup form or a placebo. The placebo beverage had two to three drops of the chlorpheniramine maleate syrup floated on the surface. After consuming the beverage, subjects waited one hour for the drug to reach an effective blood level, answered some questionnaires related to mood, and physical symptoms, and then performed the battery of performance tasks. Following the two-hour test session, subjects were immediately given a light meal and were allowed to relax for the remainder (approximately 40 minutes) of that hour. Again, the light meal was devoid of any foods that might slow drug absorption rate. During the relaxation period, subjects typically watched television, read, or joined in small group conversations. At the end of the first rest period, which was four hours after consuming their first drug/placebo beverage, subjects were administered their second drug/placebo beverage. On any specific test day, a subject received only one drug condition. That is, all beverages on any specific day either contained the drug dose or the placebo. After one hour, subjects again completed the entire battery of questionnaires and performance tasks. A light snack was served and subjects relaxed for the remainder of that hour, which was followed by the third drug/ placebo beverage, one-hour waiting period, and the third battery of questionnaires and performance tasks.

The results of this study can best be summarized by the following table, which provides an overview of the main effects and two-way interactions from the various analyses of variance. (There were no three-way interactions that were statistically significant.) What is immediately apparent from the table is that the greatest number of significant findings existed for the Drug main effect and the Shift $x$ Session interaction. The Shift and Session main effects evidenced considerably fewer significant findings, and the other two interactions had very few significant findings. Of course, the simple frequency or even magnitude of significant effects may not be the best way in which to evaluate the outcome of an investigation. However, in this case, the general trends in significant findings were not only apparent but were also consistent with prior research evidence.

Because this study attempted to explore factors that may affect safety-sensitive job performance, a broad range of tasks and self-report measures was used and a somewhat conservative examination of the results was conducted. This conservative approach possibly resulted in an over-inclusiveness with regard to significant findings. Thus, an examination of general trends in the findings is an important consideration in this study. Evidence of a factor having an influence 


\section{Summary of Significant ANOVA Effects.}

(In small italics: $0.05<\mathrm{p}<0.10$; all others: $\mathrm{p}=.05$ )

\begin{tabular}{|c|c|c|c|c|c|c|}
\hline TASK & Drug & Shift & Session & Drug $x$ Shift & Drug $x$ Session & Shift $\times$ Session \\
\hline $\begin{array}{l}\text { Spatial } \\
\text { Processing }\end{array}$ & PC & & & MNCORRT & & $\begin{array}{l}\text { MNCORRT } \\
\text { PC }\end{array}$ \\
\hline $\begin{array}{l}\text { Critical } \\
\text { Tracking }\end{array}$ & MEANL & & & & & $\begin{array}{l}\text { MEANL } \\
\text { MAXL } \\
\end{array}$ \\
\hline $\begin{array}{l}\text { Dual Task } \\
\text { (Group Lambda) }\end{array}$ & $\begin{array}{l}\text { RMS } \\
\text { CTLOSS } \\
\text { MNCORRT } \\
P C \\
\text { THRPUT } \\
\end{array}$ & & RMS & & RMS & \begin{tabular}{|l} 
RMS \\
CTLOSS \\
MNCORRT \\
PC \\
THRPUT \\
\end{tabular} \\
\hline $\begin{array}{l}\text { Dual Task } \\
\text { (Indiv. Lambda) }\end{array}$ & \begin{tabular}{|l} 
RMS \\
CTLOSS \\
MNCORRT \\
THRPUT \\
\end{tabular} & & RMS & $P C$ & & \begin{tabular}{|l} 
RMS \\
CTLOSS \\
MNCORRT \\
THRPUT \\
\end{tabular} \\
\hline $\begin{array}{ll}\text { IML } & \text { Manikin } \\
\text { Attention } & \\
\text { Switching } & \\
\end{array}$ & & MANPCX & MANPC & & & $\begin{array}{l}\text { MANCORRT } \\
\text { MANCORTX } \\
\text { MANTP } \\
\end{array}$ \\
\hline Math & \begin{tabular}{|l} 
MTHCORRT \\
MTHCORTX \\
MTHTP \\
\end{tabular} & $\begin{array}{l}\text { MTHCORRT } \\
\text { MTHCORTX } \\
\text { MTHTP } \\
\end{array}$ & & & & \\
\hline \begin{tabular}{|lr} 
NovaScan $^{T M}$ & Vec \\
FAA Test & Mem \\
\end{tabular} & VECPC & $M E M P C$ & MEMPC & & & VECCRT \\
\hline \begin{tabular}{|l|} 
Air Traffic \\
Scenarios Test \\
(ATST) \\
\end{tabular} & $D E L A Y$ & & PCDEST & & & DELAY \\
\hline $\begin{array}{ll}\text { Multi- } & \text { Monitor } \\
\text { Attribute } & \text { Conm } \\
\text { Task } & \\
\text { Battery } & \text { Track } \\
\text { (MATB) } & \text { Tanks } \\
\end{array}$ & $\begin{array}{l}\text { MONRT } \\
\text { COMCRT } \\
\text { COMER } \\
\text { TRKRMS } \\
\text { TNKMAD } \\
\end{array}$ & COMCRT & COMER & & COMCRT & \begin{tabular}{|l} 
MONRT \\
COMCRT \\
COMER \\
TRKRMS \\
TNKMAD \\
\end{tabular} \\
\hline
\end{tabular}

\begin{tabular}{|c|c|c|c|c|c|c|}
\hline \begin{tabular}{|l|} 
QUESTIONNAIRE \\
\end{tabular} & Drug & Shift & Session & Drug $x$ Shift & Drug $x$ Session & Shift $x$ Session \\
\hline $\begin{array}{l}\text { Antihistamine } \\
\text { Symptoms } \\
\text { Questionnaire }\end{array}$ & & & & & & \\
\hline $\begin{array}{l}\text { Activity State } \\
\text { Questionnaire }\end{array}$ & & $\begin{array}{l}\text { PHYSICAL } \\
\text { PREPARED }\end{array}$ & \begin{tabular}{|l} 
PHYSICAL \\
PREPARED
\end{tabular} & PHYSICAL & & $\begin{array}{l}\text { PHYSICAL } \\
\text { PREPARED }\end{array}$ \\
\hline Mood Scale II & \begin{tabular}{|l} 
ACTIVITY \\
HAPPINESS \\
\\
Overall $R T$ \\
\end{tabular} & $\begin{array}{l}\text { ACTIVITY } \\
\text { HAPPINESS } \\
\text { DEPRESSION } \\
\text { FATIGUE }\end{array}$ & $\begin{array}{l}\text { ACTIVITY } \\
\text { HAPPINESS } \\
\text { FATIGUE }\end{array}$ & $\begin{array}{l}\text { ACTIVITY } \\
\text { Overall RT } \\
\end{array}$ & & \begin{tabular}{|l} 
ACTIVITY \\
HAPPINESS \\
DEPRESSION \\
ANGER \\
FATIGUE \\
Overall RT \\
\end{tabular} \\
\hline $\begin{array}{l}\text { NASA-TLX } \\
\text { (ATST) }\end{array}$ & & & & & FRUSTRATION & \\
\hline $\begin{array}{l}\text { NASA-TLX } \\
\text { (MATB) }\end{array}$ & MENTAL & & & & & \begin{tabular}{|l} 
MENTAL \\
PHYSICAL \\
PERFORMANCE \\
FRUSTRATION
\end{tabular} \\
\hline
\end{tabular}


across the performance and self-report measures, especially if that influence is consistently negative, would raise much more concern regarding that factor than would a random pattern of significant findings. Such a case of consistent negative influence existed for two factors in this study.

The administration of chlorpheniramine maleate appeared to have fairly profound negative effects across a wide range of performance measures. Statistically significant negative effects resulted for some component of every simple, dual, and complex task that subjects performed. This trend in the results suggested that, in general, the effects of chlorpheniramine maleate were not selective across various types of tasks. Such broad-ranging effects might also support the view that the actions of this drug disrupt performance resources at a more fundamental and broad level, such as basic central nervous system structures as opposed to a higher order or more selective level.

Despite the broad ranging effects of this drug, it was possible to identify more specific aspects of performance that were disrupted by its administration. For example, chlorpheniramine maleate exposure led to degraded tracking performance on every task that had a tracking component (Critical Tracking, both variations of the Dual Task, and the MATB). Chlorpheniramine maleate exposure also led to degraded response speed in a wide range of tasks. While some of the simple tasks did not show RT differences (e.g., Spatial Processing), significantly slower response speed under chlorpheniramine maleate administration was found for five of the seven tasks that had response speed components. This result would suggest that chlorpheniramine maleate did have significant negative effects on the speed with which subjects responded to critical elements of these tasks. The negative effects on response speed of this drug were also recognized at another cognitive level. Subjects were not only significantly slowed in their processing of the performance tasks, they were also significantly slowed in their response to self-reported mood ratings. This would suggest that chlorpheniramine maleate not only caused slowing of the sensory motor components of task performance, but also caused slowing of the central processing of verbal tasks as well.
While it is clear that chlorpheniramine maleate had a negative effect on a broad range of tasks and that it negatively affected the speed with which subjects responded to many tasks, it is less clear what effect chlorpheniramine maleate had on accuracy. Of the several tasks that provided accuracy measures, only two yielded significant Drug main effects for percent correct measures. Of interest though is the fact that the two tasks yielding significant effects for accuracy were both spatial tasks in nature. It is unclear whether chlorpheniramine maleate may exert negative effects on the accuracy of tasks related to spatial ability because of its pharmacological actions on neurological centers that bear on spatial perception, or whether the effects on accuracy are more broad-ranging, but simply not pronounced enough in this study given the dosages and tasks used. Thus, the accuracy results are unclear, but the findings regarding spatial processing seem very much worth additional study, especially because this is such a critical skill for both ATCs and pilots.

The other area where many significant effects were found was the Shift $x$ Session interaction. This refers to the complex changes that occur across the three testing sessions for the Day Shift vs. the Midshift testing conditions. Once again, some component of every task showed a Shift $\mathrm{x}$ Session interaction. In general terms, the form of these interactions suggested that performance for the Midshift was better than for the Day Shift during the initial session of the work shift (Session 1), but was worse than the Day Shift for the final session (Session 3). In many cases, this was a fairly complete cross-over interaction with the shift groups appearing to differ at Session 1 and then reversing the direction of their differences by Session 3. In other cases, the performance was different between the shift groups only at the beginning or at the end of the shift. In these cases, the differences were in the same direction as the differences in the cross-over interactions. For example, in nearly all cases where performance was comparable at the beginning of the shift, performance for the Day Shift was better at the end of the shift, and in nearly all cases where performance was comparable at the end of the shift, performance during the first session was better for the Midshift. Viewed from a more general perspective, 
these results revealed that across the sessions, performance during the Day Shift generally got better and performance during the Midshift generally got worse.

From the results of the Activity State Questionnaire, it was clear that subjects reported significantly more physical symptoms during the Midshift, as compared to the Day Shift, and they reported increasing levels of physical symptoms across the testing sessions. It should be noted that, in general, these are considered negative physical symptoms, and chlorpheniramine maleate administration did not play an important role in this effect. It appears that either the Session factor has a quite different effect on the sense of physical well being of the subjects, given the shift they are working, or there might be a circadian cycle of changes in physical symptoms in the opposite direction across the two shifts. The level of negative physical symptoms declined slowly across the Day Shift. Across the Midshift, the level of negative physical symptoms increased markedly. What is interesting is that the level of physical symptoms reported by subjects at the end of the Day Shift is quite similar to the level reported by subjects when they begin the Midshift. This is typically the lowest level of the day. In fact, these two points in time represent the same time of day.

Other self-report measures provided additional findings of interest. The Mood Scale II results revealed that chlorpheniramine maleate administration resulted in feelings of being less active and less happy. The Shift factor seemed to have an even greater effect in that not only did subjects feel less active and less happy during the Midshift as compared to the Day Shift, but also they felt more depressed and fatigued. As noted previously, the size of some of these effects was not great in absolute terms. The Shift $\mathrm{x}$ Session interaction discussed above was also present for these self-reported mood measures and these mood results paralleled the findings noted for the Activity State Questionnaire-that is, Day Shift ratings suggested mood improvement across the shift, while Midshift ratings suggested mood decline across the shift.

Self-report measures of workload were not as effective in revealing significant differences as were other self-report measures, but the pattern of self-reported workload differences was interesting. The NASA-
TLX measure yielded essentially no differences due to chlorpheniramine maleate administration. This would suggest that the NASA-TLX was relatively insensitive to the antihistamine effect. On the other hand, there were several self-reported differences in workload for the Shift $x$ Session interaction. Again, the ratings of various aspects of workload were much like the ratings for mood and physical condition. Subjects generally felt that workload decreased across the Day Shift, but seemed to increase across the Midshift. In this situation, the combined influence of these factors may have been enough to cause subjects to subjectively view the workload as having changed.

The interpretation of the remaining significant findings for the Shift and Session main effects are difficult. These significant findings are clearly far fewer in number and are further clouded by the fact that the Shift $\mathrm{X}$ Session interaction was so influential. Prudence would suggest that these results be cautiously generalized. In all cases, however, these effects seemed to parallel the general findings already seen for the Drug and Shift $x$ Session factors.

Finally, there are a few additional observations that are noteworthy. First, there did not seem to be strong evidence for a pronounced negative effect as the result of combining antihistamine and shift. This was surprising because logic would suggest that a sedating drug in combination with a fatiguing work shift might lead to an even more dramatic negative effect on performance than either factor alone or than their simple additive effect. This did not appear to be the case in this study. Prudence, and perhaps simple skepticism, would suggest that this finding be further explored.

Second, the value of assessing change within a shift was self-evident in this study. The dynamics of performance and mood change across shifts were quite different, and the presence of cross-over interactions would suggest that conducting studies without repeated measures across shifts (i.e., using global measures summed across the entire shift) would obscure these very dramatic differences.

Third, asking subjects about antihistamine effects based on known side-effects does not seem like a very productive way to assess their influence, at least in the manner used in this study. Perhaps a more sensitive 
response scale might gain better results. The measure used in this study was clearly not effective for the dose and level of side-effects experienced.

Fourth, there was some limited evidence of differential sensitivity within tasks for various risk factors. For example, the Attention Switching Task provided some interesting results in addition to those already noted. This task directed the subject to perform either the Manikin Task or Mathematical Processing Task component. The Mathematical Processing Task appeared to be reasonably sensitive to the effects of chlorpheniramine maleate, while the Manikin Task appeared to be sensitive to the combined effects of Shift and Session. The Critical Tracking Task provided another possible example of differential sensitivity, and perhaps even the Spatial Processing Task. This apparent differential sensitivity, while intriguing, must be interpreted cautiously. It may be that some of these tasks do offer dependent measures that respond differentially to various risk factors. It may also be the case that other factors may have simply reduced the possibility of finding significant differences for that measure in relationship to other factors in this study.

In summary, the findings of this study suggest that chlorpheniramine maleate alone has a fairly strong negative influence on a wide range of task performance and mood measures. There also seems to be a rather complex relationship between work shift and time on the shift (Session) such that performance and mood during the Day Shift tend to get better and during the Midshift tend to get worse. No evidence was found that chlorpheniramine maleate and work shift combine in producing a multiplicative effect on performance or mood. 


\section{Workshift and Antihistamine Effects on Task Performance}

\subsection{INTRODUCTION}

Considerable concern has been raised in recent years about factors in the work place that compromise the effectiveness of workers. Of special concern for the aviation environment is the fact that shift work appears to have negative effects on sleep patterns (Akerstedt, 1990; Turek, 1986), self-reported satisfaction with working conditions (Melton, McKenzie, Smith, Polis, Higgins, Hoffman, Funkhouser, \& Saldivar, 1973), and task performance (Alluisi \& Morgan, 1982). In 1983, Schroeder and Goulden compiled a bibliography of research related to shift work. At that time, over 1300 studies had been published-a clear indication of the level of concern for this issue.

Such concern seems all the more relevant today, especially for Air Traffic Control Specialists (ATCSs) who have in the U.S. historically worked a rapid rotating shift schedule called the "2-2-1" (Melton \& Bartanowicz, 1986; Price \& Holley, 1990). This schedule involves working two afternoon shifts, followed by two morning shifts, and one "midshift" or night shift, within a five-day period. Recent laboratory and field research suggests that such schedules do disrupt sleep patterns among ATCSs in particular (Cruz \& Della Rocco, 1995; Della Rocco \& Cruz, 1995), and appear to disrupt work performance in numerous job categories (Alluisi \& Morgan, 1982). Further evidence based on a wide range of jobs in the aviation environment supports this finding (Rosekind, Gander, Miller, Gregory, Smith, Weldon, Co, McNally, \& Lebacqz, 1994).

Also of increasing concern for the aviation environment is a recent finding regarding common over-thecounter (OTC) drug use. A comprehensive survey of aviation accidents involving fatalities revealed that OTC antihistamines were among the most frequently found drugs (Canfield, Flemig, \& Hordinsky, 1995). These authors noted that "Chlorpheniramine and diphenhydramine, two antihistamines found in 68 of the pilots analyzed [i.e., fatalities], are sedative and may cause impairment of a pilot's ability to react to an emergency." While antihistamines were present in only $3.7 \%$ of the 1845 fatalities with positive toxicology results between 1989 and 1993, they presented a relatively high frequency rate compared to all other drugs. The antihistamines were also about the only drugs that had a sedating effect and were clearly disproportionately represented among all drug categories (with only common analgesics rating noticeably higher).

As early as 1968, Federal Aviation Administration (FAA) researchers had discovered that the administration of one common antihistamine, chlorpheniramine maleate, significantly reduced psychomotor performance (Higgins, Davis, Fiorica, Iampietro, Vaughan, \& Funkhouser, 1968). Equally important was the additional finding that, while both chlorpheniramine maleate and altitude had significant negative effects on psychomotor performance, there was a negative "synergistic effect" such that performance decrements under the two factors in combination were much greater than the simple sum of the decrements for each factor. This would suggest that common OTC antihistamines may pose a significant risk to the aviation community. Also of interest is the fact that the frequency of OTC drugs found in aviation fatalities has increased over $140 \%$ between 1989 and 1993 (Canfield et al., 1995). The authors of this report judiciously note that this increased frequency may be the result of improved biochemical analysis techniques or may be due in part to the broader availability and use of OTC drugs during this period.

Such findings raise serious concerns regarding the effects of shift work and OTC antihistamine use in the aviation environment. Perhaps more troubling is the possibility that antihistamines may well be used during shift work, thereby possibly compounding these negative effects. The purpose of this study was to investigate these potential effects. The implementation and completion of this study were significantly aided by the fact that the FAA was simultaneously supporting an assessment of both simple and complex performance tasks that appear to be sensitive to the effects of drugs and other risk factors, such as fatigue, alcohol, and sleep loss (see Gilliland \& Schlegel, 1997). By recruiting highly trained subjects from this prior study, considerable reductions in training costs, laboratory setup costs, implementation costs, and startup time were realized. 


\subsection{BACKGROUND}

\subsection{Shift Work}

The role of biological rhythms in regulating physiological and behavioral functioning has been extensively investigated. It has been well established that human biological functions (Aschoff, 1965, 1981; Wever, 1979, 1985) and a wide range of performance capabilities (Colquhoun, 1982; Folkard \& Monk, 1985; Kleitman, 1963) vary within a reasonably welldefined circadian cycle. As might be expected, sleep/ wake and temperature cycles also assume rhythmic patterns and interact in a complex fashion with biological and performance rhythms, all seemingly regulated by time cues (or "zeitgebers," see Rutenfranz, Aschoff, \& Mann, 1972), not all of which are fully understood (Wever, 1981). What has been well agreed upon, however, is that there appears to be a complex interaction between sleep cycles and the overall circadian pattern of human biological and behavioral functioning (Aschoff, 1981; Wever, 1981), and that either shifts in the sleep cycle (see Monk, 1990; Taub \& Berger, 1973, 1976) or sleep loss (Collins, 1976; Naitoh, 1976; Wilkinson, 1965; Williams, Lubin, \& Goodnow, 1959) can have quite negative effects on performance capability.

Of course, one factor that typically alters the sleep cycle is a change in work shift. Changing work shifts has been the subject of intense study (see Folkard, 1981; Folkard \& Monk, 1985). In fact, Schroeder and Goulden (1983) compiled over 1300 references on the topic. Change in sleep appears to be such a critical factor in degraded performance (Akerstedt, 1990) that it even exceeds sleep loss (Taub \& Berger, 1973) in its negative influence. However, despite several decades of research, it is not uncommon to find a hint of exasperation in researchers trying to unravel the myriad factors influencing the relationship between shift work and performance. Folkard (1981) reflects this in his summary statement, "At this stage all that can be concluded with certainty is that the problem of impaired night shift performance is far more complex than has been recognized in the past" (p. 301).

From a more circumscribed perspective, the influence of change in work shift on air traffic controllers has received careful investigation. As noted previously, ATC facilities typically use the 2-2-1 shift schedule, and it is generally recommended over other shift schedules (Melton \& Bartanowicz, 1986). This schedule usually results in a reduction in sleep per day across the week in comparison to a regular five-day, "straight" shift schedule (Schroeder, Rosa, Witt, \& Banks, 1995; Saldivar, Hoffman, \& Melton, 1977).

In one of the earliest studies among ATCs of the 22-1 work shift schedule compared to the straight shift schedule, Melton et al. (1973) found that heart rate was elevated during the night shift of the 2-2-1 shift, as compared to the straight shift. Other differences were only slight or inconsistent. The authors concluded that any differences between the shifts was too slight to be of serious concern. However, it was noteworthy that in the case of both shift schedules, the ratings for the midshift condition were considerably more negative than those for the day or evening shift. Melton and his colleagues also studied two different air traffic control centers and, in the course of this investigation, again confirmed that there was little evidence for differences in stress or performance due to the 2-2-1 schedule, as compared to the straight shift schedule (Melton, Smith, McKenzie, Saldivar, Hoffman, \& Fowler, 1975).

More recently, Della Rocco and Cruz (1995) investigated the effects of the 2-2-1 schedule on a variety of measures and found that the 2-2-1 schedule disrupted sleep patterns more than the straight-shift pattern, and sleep quality ratings also declined under this schedule. While their report focused on the dynamics of the sleep cycle, the authors did note that performance was degraded, but primarily on the night shift component. Cruz and Della Rocco (1995) have also reported a field study of sleep patterns among ATCs who were working various shift schedules (including straight, 2-2-1, and 2-1-2 shifts). The results of this study revealed that there was little, if any, difference between the shift schedules in terms of sleep function or quality.

In more general terms, past research suggests that the disruption of circadian cycles, especially sleep, can have a detrimental effect on performance. While changes in work shift can certainly cause circadian rhythm disruption, the degree to which various shift changes differ from one another in their effects on performance is unclear. It would appear that the myriad factors that mediate the relationship between sleep disruption and performance may cause performance decrements just as large as the differences between various shift schedules. What does seem clear, is that the midshift (or 
night shift) is the most unpopular and the most potentially degrading shift with respect to performance (Melton \& Bartanowicz, 1986).

\subsection{Antihistamines}

Antihistamine drugs act primarily by blocking the receptor sites of histamine, a depressor amine that is produced by the enzyme histidine decarboxylase acting on the amino acid histidine. Histamine is stored mainly in mast cells. When the body is irritated, the mast cells release varying amounts of histamine, which causes vasodilation and increased permeability of the blood vessel wall, the physiological precursors of common allergic reactions. The biochemical action of histamine is due to its direct binding at two known receptor sites, which are classified as either $\mathrm{H}_{1}$ or $\mathrm{H}_{2}$ sites.

The origins of antihistamine drugs can be traced to the early work of Bover and Staub (1937), although widespread therapeutic use of antihistamines did not occur until the introduction of pyrilamine maleate (Bovet, Horclois, and Walthert, 1944), which is still among the most effective antihistamines. These early antihistamines, as well as scores of others synthesized since the introduction of pyrilamine, are effective in opposing or blocking the action of histamine at the $\mathrm{H}_{1}$ receptor sites. These antihistamines are particularly effective in opposing the symptoms commonly associated with allergic reactions, but they pose a problem because one of their major side-effects is sedation. Almost all $\mathrm{H}_{1}$-blocking antihistamines cause some level of sleepiness, so much so that they form the active ingredients in common, over-the-counter sleep medications.

Recently, new generations of $\mathbf{H}_{1}$-blocking antihistamines have been introduced, as well as $\mathrm{H}_{2}$-blocking antihistamines. These are often referred to as nonsedating antihistamines, because they have far less, or nearly no, sedation side-effect. Several studies have shown that these newer, non-sedating antihistamines have few, if any, negative effects in comparison to the earlier $\mathrm{H}_{1}$-blocker antihistamines. For example, it has been shown that newer, non-sedating antihistamines have no apparent negative effects on various cognitive and psychomotor abilities (Cingi, Cingi, \& Cingi, 1990; Clarke \& Nicholson, 1978; Fink \& Irwin, 1979; Kulshrestha, Gupta, Turner, \& Wadsworth, 1978; Nesthus, Schiflett, Eddy, \& Whitmore, 1991; Nicholson \& Stone, 1982; Nicholson \& Stone, 1986; Philpot, Biegalski, \& Brooker, 1993; Reeves, Blackwell, Molina, \& Hixson, 1989; Rice \& Snyder, 1993;
Snyder \& Berg, 1990; Tharion, McMenemy, \& Rauch, 1994; Witek, Canestrari, Miller, Yang, \& Riker, 1995). They also do not appear to disrupt EEG activity (Loring \& Meador, 1989; Stephens, Caldwell, Comperatore, Pearson, \& Delrie, 1992; Tharion et al., 1994) or more basic sensory-psychophysical functions (Fink \& Irwin, 1979; Kulshrestha et al., 1978; Nicholson, Smith, \& Spencer, 1982; Nicholson \& Stone, 1982; Nicholson \& Stone, 1986), and they do not typically influence self-reported levels of subjective state (Philpot et al., 1993; Reeves et al., 1989; Tharion et al., 1994). In addition, investigations of complex job-related performance have revealed that non-sedating antihistamines do not appear to affect driving performance (Betts, Markman, Debenham, Mortiboy, \& McKevitt, 1984), marksmanship (Johnson \& McMenemy, 1989), flight simulator performance (Philpot et al., 1993; Stephens et al., 1992), or complex command, control, and communications $\left(\mathrm{C}^{3}\right)$ team performance (Eddy, Dalrymple, \& Schiflett, 1992).

Despite the lowered risk of side-effects from the new generation of non-sedating antihistamines, many people use older $\mathrm{H}_{1}$-blocking antihistamines, no doubt because they can be purchased without prescription and are prevalent in many OTC preparations. Many of these antihistamines have been shown to produce significant decrements in cognitive skills (Burns, Shanaman, \& Shellenberger, 1994; Cingi et al., 1990; Hindmarch, Kerr, \& Sherwood, 1991; Nesthus et al., 1991; Nicholson \& Stone, 1982; Nicholson \& Stone, 1986; Reeves et al., 1989; Rice \& Snyder, 1993; Snyder \& Berg, 1990; Witek et al., 1995) and basic psychomotor abilities (Cingi et al., 1990; Clarke \& Nicholson, 1978; Fink \& Irwin, 1979; Hindmarch et al., 1991; Kulshrestha et al., 1978; Nesthus et al., 1991; Nicholson, 1979; Nicholson \& Stone, 1982; Nicholson \& Stone, 1986; Reeves et al., 1989; Rice \& Snyder, 1993; Snyder \& Berg, 1990; Witek et al., 1995). Further, negative effects due to the older $\mathrm{H}_{1}$ blocking antihistamines have been shown in studies of job performance and studies of operational environments (Betts et al., 1984; Eddy et al., 1992; Johnson \& McMenemy, 1989; Stephens et al., 1992). So obvious are the risks involved in using these drugs in operational environments, a number of recent reviews have warned of their negative consequences (Meltzer, 1990, 1991; Nicholson, 1985). 
As noted previously, chlorpheniramine maleate is among the more common drugs found in fatal aircraft accidents. It is also one of the more common antihistamines used by allergy sufferers and is one of the most commonly found antihistamines in a wide variety of OTC cold, flu, and allergy medications. Chlorpheniramine maleate is a traditional $\mathrm{H}_{1}$ receptor site blocker and has moderately high sedating effects (see Goodman \& Gilman, 1990; Manning \& Gengo, 1993).

Chlorpheniramine maleate has also been shown to have fairly wide-ranging negative effects on a variety of performance abilities. Early studies by FAA researchers revealed that administration of a compound containing chlorpheniramine maleate $(4 \mathrm{mg})$ caused a significant reduction in psychomotor ability (Higgins et al., 1968), as did altitude. These researchers also studied the combined effects of altitude and the chlorpheniramine maleate compound and concluded that, "There was...a synergistic effect, so that performance under both drug $B$ [the chlorpheniramine maleate compound] and 14,000 foot altitude conditions was poorer than the sum of the two separate influences" (p. 3). These findings are particularly sobering given the recent findings of Canfield et al. (1995) regarding the incidence of chlorpheniramine maleate in fatal aircraft accidents.

In later research, Clarke and Nicholson (1978) investigated the effect of chlorpheniramine maleate ( 4 $\mathrm{mg}$ ) on visuo-motor ability, as assessed by a pursuittype tracking task, and found that chlorpheniramine maleate caused a significant degradation in performance with respect to the placebo condition. The most negative effects on performance were found approximately 1.5 hours after ingestion. This was also one of the first studies to include self-report measures of the subjects' performance effectiveness and sense of well-being. Subjects reported no perceived decline in performance ability as a result of chlorpheniramine maleate, and reported only that they were significantly less energetic than under placebo conditions. This finding was important because it suggests that those under the influence of chlorpheniramine maleate do not always recognize the degree to which their performance is compromised, even when that performance is significantly degraded. This finding must also be tempered by the fact that other researchers have reported no wide ranging negative effects of chlorpheniramine maleate $(4 \mathrm{mg})$ on performance (such as critical flicker fusion, pursuit rotor, and simple reaction time), but did find increases in subject feelings of fatigue among their subjects (Kulshrestha et al., 1978). This disparity between the effects of chlorpheniramine maleate on measures of performance and self-reported mood has been noted by several authors (Manning \& Gengo, 1993; Meltzer, 1990, 1991; Nicholson, 1985). It serves as a point of considerable concern, especially in the case when an individual has taken chlorpheniramine maleate and cognitively perceives little effect, yet demonstrates significantly degraded performance.

Chapman and Rawlins (1982) conducted a study in which subjects performed a letter cancellation task and also provided self-reported assessments of sedation level (with a simple scale ranging from "wide awake" to "almost asleep") following administration of chlorpheniramine maleate $(16 \mathrm{mg})$. The authors reported that subjects showed a significant reduction in letter cancellation ability both 2 hours and 4 hours after dosing and reported significantly more sedation as compared to a control condition. Similar results were found in a study of digit symbol substitution. Nicholson, Pascoe, Turner, Ganellin, Greengrass, Casy, and Mercer (1991) found that, in comparison to a placebo condition, subjects administered $10 \mathrm{mg}$ of chlorpheniramine maleate showed significant declines in digit symbol substitution performance, had significant reductions in sleep latency, and reported that they felt significantly more sleepy.

Lee, Lader, and Kitler (1988) studied the effects of chlorpheniramine maleate $(12 \mathrm{mg})$ on tapping and reaction time performance, as well as EEG activity and self-reported measures of mood and physical symptoms. The results of this study revealed that chlorpheniramine maleate administration led to initial increases in tapping rate and then fairly profound decreases. Reaction time was significantly slowed overall by the introduction of chlorpheniramine maleate, and EEG patterns were disrupted, causing shifts toward increasing alpha wave activity $(8.0-13.0 \mathrm{~Hz})$, a pattern commonly associated with lower activation states. Subjects in this study reported improvement in general alertness and contentedness after the administration of chlorpheniramine maleate, but also reported less ability to concentrate. It is unclear why subjects in this study would report subjective feelings that differ so from those reported in other studies, that is, either no difference as a result of chlorpheniramine maleate administration or various negative effects. The only factor that might explain this finding is the form of chlorpheniramine maleate administered in this study. 
These authors administered a sustained-release preparation noting that, "...performance impairment is less than that with standard formulations" (p.111). The authors concluded that, "...sustained-release preparations seem a useful advance on standard formulations with respect to obviating central sedative effects" (p.117).

In another study of chlorpheniramine maleate effects on EEG activity, Loring and Meador (1989) found that an $8 \mathrm{mg}$ dose caused significant slowing of the P300 cortical evoked response. The P300 response is an EEG measure that has been linked to both speed of cortical processing and sustained attention ability. These results, as well as those of Lee et al. (1988), suggest that the degrading effects that chlorpheniramine maleate seems to exert on performance may well be mediated indirectly by the suppression of midbrain $\mathrm{H}_{1}$ histaminergic receptors that play a central role in regulating general arousal level (see Goodman \& Gilman, 1990; Manning \& Gengo, 1993; Prell \& Green, 1986).

More recently, the effects of chlorpheniramine maleate $(16 \mathrm{mg})$ have been studied with respect to several cognitive and psychomotor performance tasks including digit symbol substitution, critical flicker fusion, and digit cancellation tasks (Khosla, Saha, Koul, Chakrabarti, Sankaranarayanan, \& Sharma, 1993). The data from this study are difficult to interpret due to contradictory statements by the authors, but there is at least clear evidence of impairment on several of the cognitive and psychomotor performance tasks studied, and the subjects also reported significant increases in self-reported sleepiness, tiredness and difficulty in concentrating. Witek et al. (1995) also performed a study of chlorpheniramine maleate effects on a wide range of computerized tasks including reaction time, divided attention, and hand steadiness. Chlorpheniramine maleate $(4 \mathrm{mg}$ ) was shown to degrade reaction time and resulted in higher levels of self-reported sleepiness.

Finally, one study investigated chlorpheniramine maleate effects on pilot performance. Philpot et al. (1993) examined the performance of military pilots under the influence of chlorpheniramine maleate ( 4 $\mathrm{mg}$ ) and found no negative influence on flight simulator performance, and a wide range of psychological and neuropsychological tests. The subjects did report a wide range of negative symptoms associated with exposure to chlorpheniramine maleate. The authors of this study note an important point with regard to chlorpheniramine maleate effects on performance: While many studies do report degraded performance following administration of chlorpheniramine maleate, it is possible for highly motivated subjects to overcome these negative effects and perform to competent or even exceptional levels.

In general, there is considerable evidence that chlorpheniramine maleate has the capacity to exert a negative influence on a wide range of performance capabilities and to negatively influence self-reported cognitive states. Admittedly, there is some evidence to the contrary, but the preponderance of research results suggests fairly clear evidence of detrimental effects. Equally critical is exploring the possibility of interactive effects of chlorpheniramine maleate and other risk factors.

\subsection{OBJECTIVES}

The primary objective of this project was to provide the FAA with a highly-controlled laboratory investigation addressing the effects of shift work and antihistamine administration on both simple and complex task performance. Three major issues were addressed:

(1) the effect of shift work on simple and complex task performance,

(2) the effect of multiple recommended therapeutic doses of OTC antihistamine on simple and complex task performance, and

(3) the interactive effect of shift work and an OTC antihistamine on simple and complex task performance.

\subsection{METHODOLOGY}

\subsection{Project Design}

As noted above, this research was significantly aided by the recruitment of subjects who had been thoroughly trained on simple and complex performance tasks that were believed to be sensitive to drug and other risk factor effects. The prior study is presented in its simplest form in Figure 1. The overall study consisted of four stages extending over a six-week period. The first stage lasted one week and included subject screening, pre-testing, and selection. During the second stage, which also lasted one week, subjects underwent orientation and training on several tasks that are described below. 
The third stage provided an extended series of test sessions spanning four additional weeks. The fourth stage of the study provided Specialized Investigation Periods that were typically conducted on weekends so as to minimize any influence on daily testing sessions during the week. Testing periods varied according to the requirements of the specific research protocol. The subjects in this prior study were extensively trained and practiced on a number of simple and complex performance tasks assessing a wide range of cognitive and psychomotor functions. An investigation of the learning characteristics of this group, as well as information on the reliability and stability of the performance measures across the five weeks of task performance, are available in a report by Gilliland and Schlegel (1997). The present study was conducted immediately following the conclusion of the prior study.

\subsection{Subjects}

Subjects were recruited from among those who participated in the previously mentioned study (see Gilliland \& Schlegel, 1997). From among those subjects who volunteered, 16 subjects were randomly selected to participate in the present study. Subjects were originally recruited from University of Oklahoma psychology and engineering classes, the general student body, and the Norman, Oklahoma, regional community. All subjects were male. Subjects in the present study ranged in age from 21 to 38 years with a mean of 25.9 and a standard deviation of 5.6 years. All subjects signed an Informed Consent Form approved by the University of Oklahoma Institutional Review Board-Norman Campus. A bonus payment system was used to increase motivation and study completion rate. Subjects were paid a base rate for the number of hours they participated and upon completion of the study, they were given an additional bonus for every hour of participation.

All subjects were surveyed for self-reported normal (or corrected-to-normal) vision, normal hearing, and the absence of any central nervous system stimulant or depressant medications. Due to the nature of the study, additional relevant information about alcohol, caffeine, medication, and possible drug use was obtained. On average, subjects consumed 3.8 alcoholic beverages per week, although individual consumption ranged from 0 to 18 beverages per week. Subjects reported that, on average, they drank two or three times a month with beer as the primary alcoholic beverage. Caffeine consumption was relatively low. Average coffee consumption was 2.6 cups per day. The average coffee consumption was skewed by one subject who reportedly drank two to three 12-cup pots of coffee daily. Average cola consumption was 1.5 cans per day. A detailed breakdown of subject characteristics is provided in Appendix A.

\section{-4.3 Test Battery}

Several factors were considered in selecting tasks for this study. One critical factor was the specific information processing skills necessary in typical safery-sensitive jobs, such as air traffic control or aircraft piloting. Another relevant issue was the information provided by a specific task with respect to the cognitive processes or information processing stages affected by one or more risk factors. These and other factors were taken into account in reviewing a large number of human performance task batteries. As a result, seven tasks, and four subjective scales from various sources were selected for inclusion in the study.

Brief descriptions of the RTP tests, work samples, and subjective rating scales used in the study follow. Detailed descriptions of the tasks and a list of relevant publications are provided in Appendix B.

\section{Performance Tasks}

Spatial Processing (SPA) - involves indicating whether a rotated pattern of histograms is the same as one previously presented. The test lasts 3 minutes.

Critical Tracking (TRK) - requires tracking an unstable object on the display using a trackball for 2 minutes.

Dual Task (DUL) - involves performing the Sternberg Memory Search while Tracking. The Sternberg Memory Search involves indicating whether a letter is the same as one of those in a previously memorized set. The test lasts 3 minutes.

IML Switching Task (NTI) - involves responding to 1 of 2 tasks presented simultaneously on each screen display. In the Manikin task, the subject presses a key to indicate which hand of a manikin holds a matching symbol. In the Mathematical Processing task, the subject presses a key to indicate whether a sum of three numbers is greater or less than 5 . The test lasts 4 minutes.

NovaScan ${ }^{\text {TM }}$ FAA Task (NSF) - consists of integrated responses to three tasks. For two of the tasks, stimulus screens are presented in directed attention fashion with a series of stimuli from one task 
alternating with a series of stimuli from the other task. In addition, a vigilance/attention task is performed for every stimulus screen. In the Visual Search and Vector Projection task, the subject searches for two labeled vectors, makes mental rotations of the vectors based on verbal on-screen instructions, and responds as to whether the rotated vectors would intersect either on or off the screen. In the Spatial Memory task, the subject memorizes the position and shape of a missing symbol for later comparison with the next spatial memory stimulus screen. For the Attention task, subjects look for the presence of small symbols in the corners of each screen. The test is based on a fixed number of stimuli and test time is thus a function of subject proficiency.

\section{Work Sample Tasks}

Air Traffic Scenarios Test (ATST) - an approximation of the air traffic control environment which involves the directing of planes to their destinations using altitude, speed, and heading changes. The work version of the task lasts 25 minutes.

Multi-Attribute Task Battery (MATB) - an approximation of the air crew operations environment which includes a monitoring task (a set of lights and a set of dials), an auditory communications task, a compensatory tracking task, and a resource management task involving the monitoring and control of fuel tank levels. The work version of this task lasts 40 minutes.

\section{Subjective (Self-Report) Measures}

Antihistamine Symptoms Questionnaire (AHSQ) - consists of a checklist of common side-effects associated with the use of antihistamines. This scale was rationally constructed based on side-effects listed in the medical and pharmaceutical literature. The test takes approximately 1 to 2 minutes.

Activity State Questionnaire (ACTSQ) - consists of 25 items scored on a seven-point scale, which was an expanded form of the Pennebaker Physical Symptoms Checklist to assess the current state of physical health. Subjects also responded to two questions regarding their level of preparedness for task performance. The test takes approximately 2 minutes.

Mood Scale II (MOOD) - involves pressing a numbered key on the computer keyboard to indicate the level of agreement with each of 36 descriptive adjectives to assess the current mood in the categories of activity, happiness, depression, anger, fatigue and fear. The test takes approximately 2 minutes.

NASA Task Load Index (TLX) - consists of ratings of task workload using the categories of mental demand, physical demand, temporal demand, performance, effort, and frustration. This collection of ratings was obtained following each work sample task. The ratings take approximately 1 minute. (Note: While the NASA TLX ratings were among the subjective ratings administered, they were completed by the subjects after each of the simulated work tasks and, thus, were linked logically to the workload generated by these tasks. The use of these TLX ratings can not be used as a reflection of overall workload experienced by the subject during the entire testing session.)

Table 1 presents a summary of the task codes used throughout the remainder of the report when referring to the various tasks and self-report measures.

\subsection{Equipment}

All tasks were presented on eight microcomputer workstations. Each workstation consisted of a Gateway 486-33 $\mathrm{MHz}$ processor with the necessary input devices ("Anykey" keyboard, Microsoft mouse, Kensington Expert Mouse 4.0 trackball, CH Products Flightstick, and NovaScan ${ }^{\mathrm{TM}}$ interface box). All data were recorded on these machines and on subject diskettes and then downloaded to a central data management system (Gateway 486-66 MHz) for data reduction and analysis using Microsoft Excel and SAS. In cases of emergency, this machine also served as a backup workstation. Testing was automated to allow a subject to perform the tests independently and in the minimal amount of time. Of course, multiple experimenters were present at all times to monitor the subject's safety and performance, and provide assistance, if needed. The software automatically performed all functions such as subject identification, file naming, test sequencing, and data backup.

\subsection{Test Facilities}

All testing was conducted in laboratory space located in the basement of Dale Hall at the University of Oklahoma. The testing workstations were approximately $3 \mathrm{ft}$ wide and $3 \mathrm{ft}$ deep and were located in one room (approximately $13 \mathrm{ft}$ by $20 \mathrm{ft}$ ). The stations were divided by 3 -inch thick acoustic panels. The comput- 
ers and response devices were placed on tables at the testing stations positioned at a height of approximately 28 inches.

Another room of approximately the same size served as the data reduction and project management office. The third room served as an auxiliary room for interviewing, orientation, and miscellaneous activities. All of these rooms represent modern laboratory space with centrally controlled heating and air conditioning. Temperature in the testing room was maintained at approximately $68^{\circ} \mathrm{F}$ throughout the test sessions.

\subsection{Experimental Procedure}

Data were collected on weekend test days that were conducted over a five-week period. The term "test day" will be used to refer to the actual time period on the weekend during which testing occurred. This was either during the day or during the Midshift period (as described below). The term "session" will refer to a series of tasks performed at a specific time on the test day. "Trial" will usually refer to a discrete response by a subject to the presentation of a single stimulus for any specific task. For example, subjects performed a series of trials for each of the tasks (which is referred to as a "session") three times on each test "day."

Subjects returned to the laboratory for two, twohour re-training sessions during the week before the first weekend test day. These re-training sessions were strategically introduced prior to the study and between the weekend test days to insure that subjects maintained reliable performance on the various tasks. On the first re-training day, subjects completed consent forms and questionnaires and were provided additional information regarding the antihistamine to be administered and detailed information about the testing protocol. They were also required to sign agreements to comply with restrictions on their transportation and activity after testing sessions during which they might be administered antihistamines. All subjects were also scheduled for one additional retraining day in the week prior to the first weekend test day, and were assigned to testing groups for the coming weekends.

Testing days were conducted on four weekends. A holiday fell in the middle of the sequence of weekends, and thus two successive weekends with testing days were followed by a holiday weekend, which was in turn followed by two more successive weekends with test days. Two of the weekends involved "Day Shift" testing days and two weekends involved "Midshift" testing days. Testing days conducted under the Day Shift condition began on Saturday mornings at 8:00 a.m. (or 10:00 a.m., see group sequence below) and ended 11 hours later. Testing days conducted under the Midshift condition began on Friday evenings at 8:00 p.m. (or 10:00 p.m., see group sequence below) and also lasted 11 hours. Day Shift and Midshift testing conditions were randomly assigned to the four weekend test days. Subjects were randomly assigned to counterbalanced orderings of the antihistamine-placebo conditions on both Day Shift and Midshift test days.

Because the laboratory contained eight microcomputer workstations, the 16 subjects were randomly assigned to one of two groups. During each testing day, one group of subjects began the experimental protocol two hours after the other group to avoid conflicts in using the workstations. In this manner, the experimental protocol was carefully structured to provide complete and identical test session sequences for both groups of eight subjects (see Table 2). The experimental protocol for each test day required eleven hours and included three, standard OTC doses of chlorpheniramine maleate (or placebo) at recommended 4-hour intervals followed by testing sessions on the various performance tasks.

The protocol included the following series of activities. Subjects were advised to eat only light meals well in advance of the initial test session and to avoid any foods that might slow drug absorption rate (e.g., high fat foods or dairy products). On each test day, subjects entered the laboratory, completed some general questionnaires, and were given a beverage containing approximately $6 \mathrm{oz}$ of fruit juice, $1 \mathrm{oz}$ of crushed ice, and either $4 \mathrm{mg}$ of ChlorTrimeton ${ }^{\circledR}$ brand chlorpheniramine maleate in syrup form or a placebo. The placebo beverage had two to three drops of the chlorpheniramine maleate syrup floated on the surface. The fruit juice beverage seemed to mask very effectively the presence of the drug, and any slight difference in aroma was equalized in the placebo beverage by the few drops of the drug syrup that were floated on top.

After consuming the beverage, subjects waited one hour for the drug to reach an effective blood level, answered some questionnaires related to mood, and physical symptoms, and then performed the battery of performance tasks. Following the two-hour test session, subjects were immediately given a light meal and were allowed to relax for the remainder (approximately 40 minutes) of that hour. Again, the light meal was 
devoid of any foods that might slow drug absorption rate. During the relaxation period, subjects typically watched television, read, or joined in; small group conversations. At the end of the first rest period, which was four hours after consuming their first drug/placebo beverage, subjects were administered their second drug/placebo beverage. On any specific test day, a subject received only one drug condition. That is, all beverages on any specific day either contained the drug dose or the placebo. After one hour, subjects again completed the entire battery of questionnaires and performance tasks. A light snack was served and subjects relaxed for the remainder of that hour, which was followed by the third drug/placebo beverage, onehour waiting period, and the third battery of questionnaires and performance tasks.

When subjects completed their third testing session for the day, they were escorted to their homes. Due to the potential sedating nature of antihistamines, no subject was allowed to drive from the laboratory or leave for activities that would involve physical risk or the use of machinery. At the end of the fourth and final test day, subjects were paid for their involvement in the study and, once again, were escorted to their homes.

During the course of the study, there were a total of 18 testing sessions (6 re-training sessions and 12 drug sessions). For all testing sessions, subjects first performed the simple single and dual-type performance tasks and then performed longer trials of both the ATST (25 min) and MATB (40 min) complex tasks. Nine different orders of the simple tasks were developed to minimize interference between consecutive tasks (e.g., hand fatigue from consecutive TRK and DUL trials). For each subject, these orders were randomly assigned in two blocks (i.e., each order was used once in the first nine sessions and once in the second nine sessions). For a given subject, the same order was never presented on consecutive sessions.

The order of the ATST and MATB tasks was alternated, with either ATST performed before MATB (order 1) or vice versa (order 2). For the first 16 sessions, the orders were blocked in sets of 4 sessions. The order for the first session was randomly selected and this specified the order of the remaining three sessions in the block. For example, if the first session was order 1 , then the order of the four sessions in the block was 1-2-2-1. On the other hand, if the first session was order 2 , then the order of the four sessions in the block was 2-1-1-2. The last two sessions used randomly assigned orders balanced across subjects and across sessions within subjects.

For the ATST task, six different 25-minute scenarios involving 45 planes were used by all subjects. For a given subject, the same scenario was used for the first re-training session and for the third session on each drug test day (i.e., for four specific sessions) to enable a baseline comparison across all treatment conditions. The remaining scenarios were randomly assigned to the other sessions with a restriction prohibiting the assignment of the same scenario to two consecutive sessions for the same subject.

There were five unique scripts for the MATB task. As with ATST, the same script was used for the first retraining session and for the third session on each drug test day. The remaining scripts were randomly assigned to the other sessions with the restriction prohibiting the assignment of the same script to two consecutive sessions for the same subject.

During each session, two versions of the Dual Task were performed by each subject in order to make the task more sensitive to variations in subject ability. One version (DULI) used an individualized lambda value set to $70 \%$ of the average of the subject's maximum lambda values for training Sessions 7 through 10 of the TRK task. The other version (DULG) used a group lambda value which was established as the average of all subjects' individualized lambda's. The group lambda value was set at 3.7. Table 3 presents the individualized lambda values for each subject.

\subsection{RESULTS}

This investigation can be described as having a multifactorial design. The main effects were Drug (antihistamine or placebo), Shift (Day Shift or Midshift), Session (testing sessions across the Shift period, that is, first, second, or third testing session within each test day), and Group (first or second group within each test day). An initial analysis of the data revealed no significant findings for the Group main effect suggesting that there were no differences due to the two-hour offset in group starting time. Therefore, the data were collapsed across this variable, and all analyses reported below are based on a totally withinsubjects, Drug x Shift $\mathrm{x}$ Session ANOVA approach. Results of these analyses for each task are presented below. 
When exploring phenomena that may have implications for human safety, additional concern should be exercised in statistical testing. Setting the alpha level for statistical significance constitutes a tradeoff between the probabilities of Type I and Type II errors. Traditional alpha levels of $p=.05$ or .01 may be favored by some readers, but these are merely conventional alpha levels that must be considered within the context of the investigation and the meaning of experimental results for broader issues. It could be argued that in situations involving human safety, protecting against Type II errors should take priority. That is, it is possible that by chance alone a truly significant effect will not be detected, which in this case would mean that an important potentially hazardous drug effect might go undetected due to a fairly stringent decision rule for statistical significance. It was decided to make it somewhat more difficult for this to occur in this study by establishing alpha at $p=.10$. The authors recognize that this has the simultaneous effect of increasing the probability of a Type I error or "false alarm" - detecting an apparent significant finding that is not truly significant. However, the authors believe that where human safety may be involved, the slightly higher probability of a false alarm has less potential risk than failing to detect real threats to safety.

\subsection{Data Reduction}

The procedure for data reduction involved several phases. Raw and summary data files from the individual subject PC diskettes and workstation hard drives were transferred to the Gateway $486 / 66 \mathrm{MHz}$ data management computer. Statistical Analysis System (SAS) DATA step input programs were used to extract the data from the summary files and to create individual SAS databases for each task. The SAS UNIVARIATE procedure was used to provide extensive descriptive statistics for each dependent variable. These analyses were reviewed for questionable data points that could be the result of procedural errors or data outliers. Very few outlier data points were removed prior to the summaries and analyses. The few deleted observations were due to identifiable hardware, software, or subject errors. Data points in question were corrected where possible and removed when necessary. Appendix D presents means and standard deviations for all main effects (Drug, Shift, Session) and two-way and three-way interactions for the major performance task measures and subjective questionnaire measures.

\subsection{ANOVA Results by Task}

\section{Spatial Processing Task}

The primary significant effect for the mean response time for Spatial Processing correct responses (MNCORRT) was a Shift $\mathrm{x}$ Session interaction, $F(2$, $30)=4.64, p=.02$. This interaction was due to the fact that while MNCORRT was somewhat faster during the Midshift, as compared to the Day Shift, for Sessions 1 and 2, this trend was reversed for Session 3 (see Figure 2). The MNCORRT measure also had a significant Drug X Shift interaction, $F(1,15)=3.52$, $p=.08$. Response time was faster for subjects under antihistamine exposure during the Midshift (as compared to the Day Shift), but faster for subjects under the placebo condition during the Day Shift (as compared to the Midshift).

A main effect for Drug was found for percent correct (PC) for the Spatial Processing task, $F(1,15)=4.77$, $p=.04$. As illustrated in Figure 3, PC for the Antihistamine condition $(M=92.56 \%, S D=6.78 \%)$ was significantly lower than PC for the Placebo condition $(M=94.39 \%, S D=4.89 \%)$. The Shift $\times$ Session interaction was also significant for the PC measure, $F(2,30)=5.05, p=.01$. This interaction effect was apparently due primarily to the fact that the Midshift condition had a lower PC than the Day Shift condition during the first session as compared to the second and third sessions. No other main effects or interactions. were significant for PC.

\section{Critical Tracking Task}

Antihistamine administration had a significant effect on the mean of the lambda's at control loss (MEANL), $F(1,15)=8.33, p=.01$, but not on the maximum lambda during the trial (MAXL). Figure 4 reveals that across all conditions, antihistamine had the effect of reducing MEANL (overall effect: antihis; tamine $M=5.11, S D=.89$; placebo $M=5.43, S D_{3}$ .79). A lower lambda is associated with poorer tracking performance.

The Shift $\mathrm{x}$ Session interaction was significant fol both MEANL, $F(2,30)=6.85, p=.004$ (see Figurs 4), and MAXL, $F(2,30)=11.87, p=.0002$ (see Figurs. 5). In both cases, the Midshift condition resulted if better tracking performance than the Day Shift durin Session 1, but by Session 3 this relationship reversed 


\section{Dual Task - Group Lambda}

Tracking. There was both a significant Drug main effect, $F(1,15)=9.69, p=.007$, and Session main effect, $F(2,30)=3.82, p=.03$, for the root mean square etror measure (RMS) for the tracking component of this Dual Task. Figure 6 presents results for the RMS tracking performance measure. Antihistamine administration led to poorer tracking performance ( $M$ $=49.0, S D=16.5)$, as compared to the placebo condition $(M=43.8, S D=16.8)$. The significant Session main effect suggested that there was also a slight, but statistically significant, decrease in performance based on higher RMS error across the three test sessions. There were also significant Drug $\mathrm{x}$ Session, $F(2,30)=3.56, p=.04$, and Shift $\mathrm{x}$ Session, $F(2,30)$ $=13.11, p=.0001$, interactions. Again, Figure 6 reveals that, when exposed to antihistamine, subjects worsened in performance from Session 1 to Session 2, and then showed a small amount of improvement during Session 3-a very minor inverted-U trend. During placebo conditions, subjects showed a very slight decrease in performance across the three test sessions. The Shift $x$ Session effect revealed a cross-over type of interaction. Subjects performed better during Session 1 in the Midshift condition, about equally well in both the Day Shift and Midshift during Session 2, and then performed worse in the Midshift condition during Session 3.

The Drug main effect for the control losses measure (CTLOSS) revealed the same general trend as that seen for RMS error, $F(1,15)=4.02, p=.06$. At first glance, Figure 7 might suggest an even greater effect than that seen for RMS error, but the low amount of variation in RMS error and the greater degree of variation in CTLOSS accounts for the lack of a higher significance level in the CTLOSS measure. Nonetheless, antihistamine did appear to have a fairly pronounced negative effect on CTLOSS rate across conditions. Under antihistamine, control losses were approximately doubled during the Day Shift and increased $30-40 \%$ during Sessions 1 and 2 for the Midshift. There was, in addition, a significant Shift $\mathrm{x}$ Session interaction for CTLOSS, $F(2,30)=4.12, p=.03$. This effect suggested that subject performance during the Day Shift was poorer than during the Midshift for Sessions 1 and 2 , and then became considerably better than Midshift performance for Session 3. A closer inspection of the data raises some serious questions, however. The obvious deleterious effect seen as a result of antihistamine administration may have artificially inflated the Day Shift CTLOSS rate. Comparison of the placebo condition data for those same sessions would suggest that performance under the two shifts was perhaps the same during Sessions 1 and 2, but that the Midshift during Session 3 showed some degradation in performance.

Memory Search. Analysis of mean correct response time (MNCORRT), shown in Figure 8, yielded a significant Drug main effect, $F(1,15)=6.40, p=$ .02 , and a significant Shift $\mathrm{x}$ Session interaction, $F(2$, $30)=3.07, p=.06$. Antihistamine administration significantly slowed response speed (antihistamine, $M$ $=685 \mathrm{msec}, S D=243 \mathrm{msec}$; placebo, $M=637 \mathrm{msec}$, $S D=202 \mathrm{msec}$ ). Figure 8 also reveals the apparent cross-over interaction of Shift $x$ Session. Subjects performed worse during the Day Shift than the Midshift at Session 1, and better during the Day Shift than the Midshift at Session 3.

Similar trends for the Drug main effect, $F(1,15)=$ $4.11, p=.06$, and the Shift $\mathrm{X}$ Session interaction, $F(2$, $30)=2.84, p=.07$, were seen for the percent correct (PC) measure. As seen in Figure 9, antihistamine administration appeared to reduce percent correct. The PC measure also tended to be slightly poorer during the Day Shift for Sessions 1 and 2, but then somewhat better during the Midshift for Session 3. The composite throughput measure (THRPUT) followed the same pattern as the MNCORRT and PC measures. In this case, both the Drug main effect, $F(1$, $15)=7.13, p=.02$, and the Shift $\mathrm{X}$ Session interaction, $F(2,30)=11.14, p=.0002$, were significant (cf. Figure 10).

\section{Dual Task - Individual Lambda}

Tracking. Results for the Dual Task - Individual Lambda tracking measures were nearly identical to the Group Lambda version of this task reviewed above. Figures 11 and 12 present the results for the RMS error and CTLOSS measures, respectively. As with the Group Lambda version of this task, there was a significant Drug main effect, $F(1,15)=6.95, p=.02$, for RMS error. Again, antihistamine administration led to poorer tracking performance $(M=47.1, S D=13.9)$, as compared to the placebo condition $(M=42.5, S D$ $=13.4)$. A significant Session main effect, $F(2,30)=$ $5.24, p=.01$, revealed a slight increase in RMS error across the three test sessions. There was also a significant Shift $\mathrm{x}$ Session, $F(2,30)=15.32, p=.0001$, interaction. From Figure 11, the cross-over type of interaction was evident once more. Subjects per- 
formed better during Session 1 in the Midshift condition, about equally well in both the Day Shift and Midshift during Session 2, and then performed worse in the Midshift condition during Session 3.

Also replicated was the significant Drug main effect for the control losses measure (CTLOSS), $F(1,15)=$ $3.50, p=.08$, which again revealed the same general trend as that seen for RMS error. Like the significant Drug main effect for CTLOSS in the Group Lambda version of this task, antihistamine appeared to have the effect of about doubling control losses during the Day Shift. There was also a significant Shift $\mathrm{x}$ Session interaction for CTLOSS, $F(2,30)=5.70, p=.008$. Once again, subject performance during the Day Shift was poorer than during the Midshift for Sessions 1 and 2 , and then became considerably better than Midshift performance for Session 3. Performance during the Midshift for Session 3 showed clear degradation compared to the first two sessions.

Memory Search. Figures 13, 14, and 15 present data from the Memory Search measures: mean correct response time (MNCORRT), percent correct (PC), and throughput (THRPUT), respectively. A significant Drug main effect, $F(1,15)=6.76, p=.02$, and a significant Shift $\mathrm{x}$ Session interaction, $F(2,30)=$ 8.62, $p=.001$, were found for the MNCORRT measure. Antihistamine administration significantly slowed response speed (antihistamine, $M=662 \mathrm{msec}$, $S D=223 \mathrm{msec} ;$ placebo, $M=621 \mathrm{msec}, S D=183$ $\mathrm{msec}$ ), just as it did in the Group Lambda version of the task. Figure 13 also reveals the cross-over interaction of Shift $x$ Session. Subjects performed worse during the Day Shift than during the Midshift at Sessions 1 and 2, and better during the Day Shift than the Midshift at Session 3.

A significant effect for the Drug $x$ Shift interaction, $F(1,15)=3.72, p=.07$, was seen for the percent correct (PC) measure. While difficult to envision from Figure 14, antihistamine administration reduced percent correct more during the Midshift than during the Day Shift. Not surprisingly, the throughput measure (THRPUT) presented in Figure 15 followed much the same pattern as the MNCORRT measure. Both the Drug main effect, $F(1,15)=6.47, p=.02$, and the Shift $x$ Session interaction, $F(2,30)=15.29$, $p=.0001$, were significant. There was overall better performance under the placebo condition, and better performance for the Midshift condition during Ses, sions 1 and 2, with poorer performance for the Midshif condition during Session 3.

\section{Switching Task}

Manikin Task. Figure 16 presents the respons. time variable (MANCORRT) for the Manikin Task The only significant finding for this measure was Shift $\mathrm{x}$ Session cross-over interaction, $F(2,30)=8.65$ $p=.001$. Response speed was faster for the Midshiff during Sessions 1 and 2, as compared to the Day Shift and appreciably slower for the Midshift during Sessio 3. The mean correct response time for transition trial (those trials immediately preceded by a trial from th alternate task) was the MANCORTX measure. Th analysis of this measure yielded a significant cross-ove Shift $\mathrm{x}$ Session interaction, $F(2,30)=4.95, p=.01$ Figure 17 reveals that the MANCORTX score fo Sessions 1 and 2 was slightly faster for the Midshi conditions. The MANCORTX score improved Session 3 during the Day Shift, and worsened Session 3 during the Midshift.

The percent correct measure (MANPC) had significant main effect for Session, $F(2,30)=2.87$, $=.07$. The PC measure showed a very slight decreat across the three sessions (see Figure 18). The percets correct transition measure (MANPCX) analysis yielde" a significant Shift main effect, $F(1,15)=3.82, p=.0$ The MANPCX scores were just slightly better durin the Midshift (Day Shift, $M=98.12 \%, S D=3.16 \%$ Midshift, $M=98.79 \%$ msec, $S D=2.53 \%$; see Figut 19). The analysis of the throughput measure (MANT) yielded a significant cross-over Shift $\mathrm{x}$ Session intere tion, $F(2,30)=7.98, p=.002$. The results present in Figure 20 illustrate that the subjects' throughp? scores improved across sessions during the Day Shi but generally declined across sessions during to Midshift.

Mathematical Processing Task. Analysis of response time variable for Mathematical Processit (MTHCORRT) yielded a significant main effect Drug, $F(1,15)=10.68, p=.005$, and a significa main effect for Shift, $F(1,15)=5.73, p=.03$. Fig 21 revealed that antihistamine exposure led to overall reduction in response speed (antihistamine, = $2096 \mathrm{msec}, S D=679 \mathrm{msec}$; placebo, $M=19$ $\mathrm{msec}, S D=663 \mathrm{msec}$ ). Also, there were slightly fa 
response speeds during the Midshift, as compared to the Day Shift (Day Shift, $M=2076 \mathrm{msec}, S D=675$ msec; Midshift, $M=2015 \mathrm{msec}, S D=670 \mathrm{msec}$ ). Analysis of response time for correct Mathematical Processing transition trials (MTHCORTX) yielded a significant main effect for Drug, $F(1,15)=11.52, p$ $=.004$, and a significant main effect for Shift, $F(1,15)$ $=3.99, p=.06$ (see Figure 22). Subjects had faster transition response times during the placebo condition, as compared to the antihistamine condition (antihistamine, $M=2268 \mathrm{msec}, S D=738 \mathrm{msec}$; placebo, $M=2165 \mathrm{msec}, S D=742 \mathrm{msec}$ ). Note also the relatively slower times for transition trials $(M=$ $2216 \mathrm{msec})$ in comparison with the overall response time ( $M=2046 \mathrm{msec}$ ).

No significant differences were found for the Mathematical Processing PC measure (MTHPC; see Figure 23) or for the Mathematical Processing PC transition measure (MTHPCX; see Figure 24). For Mathematical Processing throughput (MTHTP), there was a significant Drug main effect, $F(1,15)=11.46, p=$ .004 , and a significant main effect for Shift, $F(1,15)$ $=3.78, p=.07$. These data are presented in Figure 25 . Subjects had greater throughput scores in the placebo condition than in the antihistamine condition (antihistamine, $M=30.9, S D=10.0$; placebo, $M=32.6$, $S D=10.4)$.

The analysis of Switching Task data yielded three measures with both Drug and Shift main effects (MTHCORRT, MTHCORTX, and MTHTP) and three measures with significant Shift $\mathrm{x}$ Session interaction effects (MANCORRT, MANCORTX, and MANTP).

\section{NovaScan ${ }^{\mathrm{TM}}$}

Vector Projection Task. Vector projection correct response times (VECCRT) are presented in Figure 26. The ANOVA identified only a significant Shift $\mathrm{x}$ Session interaction, $F(2,30)=5.99, p=.006$. In comparison to their Midshift performance, subjects responded slower during the Day Shift at Session 1, about the same at Session 2, and considerably faster at Session 3.

Analysis of the percent correct measure (VECPC) yielded a significant Drug main effect, $F(1,15)=$ $13.60, p=.002$ (see Figure 27). In general, the introduction of antihistamine resulted in a reduced PC measure across all sessions and shifts (antihista- mine, $M=91.43 \%, S D=10.84 \%$; placebo, $M=$ $93.26 \%, S D=11.05 \%)$.

Continuous Spatial Memory Task. No significant differences were found for the measure of correct response time for this task (MEMCRT; see Figure 28). The analysis of percent correct (MEMPC) yielded a significant main effect for Session, $F(2,30)=3.71, p$ $=.04$. From Figure 29, it is evident that MEMPC declined somewhat from Session 1 to Session 2, and then recovered slightly at Session 3 . There was also a significant main effect for Shift, $F(1,15)=3.42, p=$ .08 , for PC. The PC measure was slightly higher during the Day Shift compared to the Midshift.

Attention Task. The analysis of percent correct on the Attention Task yielded no significant differences. This was probably due to the obvious ceiling effect observed for this measure (see Figure 30).

In summary, one NovaScan ${ }^{T M}$ task measure yielded a significant main effect for Drug (VECPC) and another measure demonstrated significant Session and Shift effects (MEMPC). One other measure had a significant Shift $\mathrm{x}$ Session interaction (VECCRT).

\section{Air Traffic Scenarios Test}

Two measures of the ATST task yielded significant differences. The analysis of the percentage of planes arriving at the correct destination (PCDEST) yielded a significant main effect for Session, $F(2,30)=8.39$, $p=.001$. Data presented in Figure 31 revealed that PCDEST improved across the sessions.

The analysis of total delay time to destination (DELAY) revealed a significant Shift $\mathrm{x}$ Session interaction, $F(2,30)=7.56, p=.002$, and a significant main effect for Drug, $F(1,15)=3.36, p=.09$. From Figure 32 it can be seen that delay decreased from Session 1 to Sessions 2 and 3 for subjects during the Day Shift, and increased from Session 1 to Sessions 2 and 3 for the Midshift. Except for Session 1 on the Midshift, the introduction of antihistamine increased the delay measure.

\section{Multi-Attribute Task Battery}

Monitoring Task. Figure 33 presents the data for the average of the two monitoring response time variables (MONRT). The analysis of these data resulted in a significant Drug main effect, $F(1,15)=4.77$, $p=.04$, and a significant Shift $\mathrm{x}$ Session interaction, $F(2,30)=8.28, p=.001$. Antihistamine exposure 
resulted in longer response times across all conditions (antihistamine, $M=3.18 \mathrm{sec}, S D=1.12 \mathrm{sec}$; placebo, $M=2.91 \mathrm{sec}, S D=.94 \mathrm{sec}$ ). In addition, a cross-over Shift $x$ Session interaction was demonstrated because, in comparison to Midshift performance, subjects responded more slowly in the Day Shift at Session 1, about the same at Session 2, and more rapidly at Session 3.

No significant differences were found in the number of response time-outs and false alarm errors for lights and dials combined (MONER). Results for this measure are presented in Figure 34.

Communications Task. Figure 35 presents data for mean response time for correct responses (COMCRT) to the Communications Task. The analysis of this variable yielded a significant Shift main effect, $F(1,13)$ $=5.52, p=.04$, and a significant Shift $\mathrm{x}$ Session interaction, $F(2,26)=5.23, p=.01$. Subjects responded faster during the Day Shift compared to the Midshift, and while they responded about equally fast during Session 1, they responded more quickly during the Day Shift for Sessions 2 and 3. Significant effects were also found for the Drug main effect, $F(1,13)=$ $3.42, p=.09$, and the Drug $\times$ Session interaction, $F(2$, 26) $=2.73, p=.08$. Antihistamine appeared to slow response speed overall (antihistamine, $M=3.82 \mathrm{sec}$, $S D=1.74 \mathrm{sec}$; placebo, $M=3.60 \mathrm{sec}, S D=1.79 \mathrm{sec}$ ), especially during Sessions 2 and 3.

The total number of errors in the Communications Task (COMER) is plotted in Figure 36. The analysis of this variable yielded a significant main effect for Drug, $F(1,15)=6.22, p=.02$, and a significant main effect for Session, $F(2,30)=3.85, p=.03$. Subjects generally committed more errors when exposed to antihistamine (antihistamine, $M=5.38, S D=6.23$; placebo, $M=4.53, S D=6.03$ ), and committed fewer errors during Session 1 than during Sessions 2 or 3.

Tracking Task. Root mean square error (TRKRMS) scores for the Tracking Task data are presented in Figure 37. The analysis of these data identified two significant effects. There was a significant main effect for Drug, $F(1,15)=5.13, p=.04$, and a significant Shift $x$ Session interaction, $F(2,30)=5.49, p=.01$. Tracking performance while exposed to antihistamine was generally worse (antihistamine, $M=54.50, S D=$ 20.97; placebo, $M=50.48, S D=20.32$ ). While tracking performance remained fairly stable across sessions for the Day Shift, it worsened considerably across sessions for the Midshift.
Resource Management Task. No large differences were found for the mean absolute deviation from 2500 units for Tanks A and B combined (TNKMAD). However, two effects were found to be statistically significant: the Drug main effect, $F(1,15)=3.27, p$ $=.09$, and the Shift $\mathrm{x}$ Session interaction, $F(2,30)=$ $2.88, p=.07$. Figure 38 presents these data. Antihistamine typically increased the magnitude of the average absolute error (antihistamine, $M=238.35, S D=$ 222.77; placebo, $M=214.56, S D=166.01$ ), and while subjects were equally effective in controlling the tank levels during the Day Shift and Midshift for Sessions 1 and 2, they were much more effective in controlling the tank levels during the Day Shift for Session 3.

- Overall, the analysis of the MATB data provided numerous significant effects for a variety of measures. Of the six major dependent measures, five yielded a significant main effect for the Drug factor. In addition, five variables had significant Shift $x$ Session interaction effects, and two other variables had significant main effects for either Shift or Session. The MATB provided a generous number of variables that appeared to be responsive to manipulations of the independent variables within this study.

\subsection{Subjective (Self-Report) Measures}

Subjects provided self-report measures of current antihistamine symptoms (AHSQ), general physical symptoms (ACTSQ), and predominant emotional state (MOOD) during each test session. Also during the test session, subjects rated the subjective workload using the NASA Task Load Index (TLX) associated with each of the two work simulation tasks (ATST and MATB). Below is a summary of the results for each of: these measures.

\section{Antihistamine Symptoms Questionnaire}

Analysis of the AHSQ responses did not yield any significant main effects or interactions. Evidently, the level of symptomology experienced by the subjects asf a result of antihistamine exposure was not sufficient to cause significant changes acknowledged at the level of a self-report measure (see Figure 39). However, th mean scores for the Drug main effect were in the expected direction, demonstrating higher levels of symptoms for the antihistamine condition compared to the placebo condition (antihistamine, $M=3.08$, SE $=2.56$; placebo, $M=2.84, S D=2.82$ ). 


\section{Activity State Questionnaire}

Figure 40 presents the data from the Activity State Questionnaire (ACTSQ). This questionnaire was included as a general measure of physical symptoms experienced by the subjects. The questionnaire includes an expansion of the Pennebaker Symptom/ Emotion questionnaire (Pennebaker, 1982). The variable PHYSICAL represents this general scale of physical symptoms. The analysis of this scale yielded several significant findings. There was a significant Shift main effect, $F(1,15)=6.92, p=.02$, and a significant Session main effect, $F(2,30)=7.05, p=.003$. Subjects reported a significantly higher level of physical symptoms during the Midshift $(M=34.44, S D=13.20)$ as compared to the Day Shift $(M=32.81, S D=13.47)$, and subjects reported a significant increase in physical symptoms across the sessions.

In addition, the Drug $\mathrm{x}$ Shift, $F(1,15)=8.04, p=$ .01 , and Shift $\mathrm{x}$ Session, $F(2,30)=13.20, p=.0001$, interactions were also significant. On the average, subjects reported a smaller increase in physical symptoms from the Day Shift to the Midshift in the antihistamine conditions, but reported a marked increase in physical symptoms from the Day Shift to the Midshift in the placebo conditions. The Shift $x$ Session interaction was represented by the fact that subjects reported approximately the same level of physical symptoms across sessions during the Day Shift, but reported a marked increase in physical symptoms across sessions during the Midshift.

The ACTSQ also provided subjects the opportunity to rate their general level of perceived preparedness for performing the tasks in that session. The PREP scale score, represents the subjects' responses (see Figure 41) for this measure. There were two significant main effects for the PREP measure. The Shift main effect was significant, $F(1,15)=5.24, p=.04$, as was the Session main effect, $F(2,30)=5.94, p=.007$. Subjects reported a significantly higher level of preparedness for task performance during the Day Shift $(M=8.77, S D=2.20)$, as compared to the Midshift $(M=8.08, S D=1.93)$, and subjects reported a significant decrease in the level of perceived preparedness across the sessions, especially during the Midshift.

\section{Mood Scale II}

Subjects also reported their moods by responding to adjectives on the Mood Scale II using a 3-point scale before completing the tasks during each session. A response of " 1 " indicated that the subject did not feel that the adjective described their current mood, while a response of " 3 " indicated that the adjective adequately described the subject's mood. The adjectives are divided into six categories (Activity; Happiness, Depression, Anger, Fatigue, and Fear). In addition, the time taken to respond to each item is also recorded (RTALL).

Activity. Analysis of the Activity scale (see Figure 42) resulted in a number of significant findings. The Drug main effect, $F(1,15)=9.79, p=.007$, was significant. Ratings of activity level during the antihistamine condition were significantly lower $(M=1.71$, $S D=0.41)$ than ratings during the placebo condition $(M=1.83, S D=0.46)$. The Shift main effect was also significant, $F(1,15)=4.63, p=.05$. Ratings of activity level during the Day Shift $(M=1.85, S D=0.46)$ were significantly higher than activity ratings during the Midshift $(M=1.70, S D=0.42)$. The analysis also yielded a significant Session main effect, $F(2,30)=$ $10.80, p=.0003$. Activity level declined across the three sessions. In addition to the main effects, there were also significant Drug $\mathrm{x}$ Shift, $F(1,15)=5.78, p$ $=.03$, and Shift $x$ Session, $F(2,30)=8.98, p=.0009$, interactions. Subjects rated their activity level during the Day Shift under the placebo condition as more active than any of the other conditions (i.e., Midshiftplacebo, or either antihistamine condition). Subjects also rated their activity level fairly constant across Day Shift sessions, but rated their activity level progressively lower across sessions during the Midshift.

Happiness. The results for the Happiness scale (see Figure 43) were very similar to those for the Activity scale. Significant main effects were found for Drug, $F(1,15)=7.71, p=.01$, Shift $F(1,15)=9.05, p=$ .009 , and Session, $F(2,30)=10.27, p=.0004$. A significant Shift $\mathrm{x}$ Session interaction was also found, $F(2,30)=12.47, p=.0001$. Subjects rated themselves happier during the placebo condition (antihistamine, $M=1.99, S D=0.52$; placebo, $M=2.17, S D=0.55$ ) and during the Day Shift (Day Shift, $M=2.17, S D=$ 0.48 ; Midshift, $M=1.99, S D=0.59$ ). Happiness declined across the sessions. The significant Shift $\mathrm{x}$ Session interaction revealed that subjects rated their happiness level fairly constant across sessions during the Day Shift, but rated their happiness progressively lower across sessions during the Midshift.

Depression. Feelings of depression seemed to be less affected by the manipulations in the study, although there was a main effect for Shift, $F(1,15)=7.65, p=$ .01 , and a Shift $\mathrm{X}$ Session interaction, $F(2,30)=3.56$, 
$p=.04$ (see Figure 44). Subjects rated themselves as slightly more depressed during the Midshift, as compared to the Day Shift, and showed a slight elevation in depression across sessions during the Midshift, while showing no change during the Day Shift. However, these changes were generally in the range of 0.03 of a scale point, which are probably not meaningful differences.

Anger. The Anger scale analysis yielded only a significant Shift $x$ Session interaction, $F(2,30)=5.89$, $p=.007$ (see Figure 45). In general, subjects reported an increase in anger feelings across the Midshift sessions, but not for the Day Shift sessions. Again, the absolute shift in anger was not particularly large (about 0.09 of a scale point).

Fatigue. The Fatigue scale yielded both Shift, $F(1$, $15)=8.57, p=.01$, and Session main effects, $F(2,30)$ $=10.15, p=.0004$, and a Shift $\mathrm{x}$ Session interaction, $F(2,30)=23.17, p=.0001$ (see Figure 46). Subjects reported greater fatigue during the Midshift (Day Shift, $M=1.47, S D=0.50$; Midshift, $M=1.64, S D$ $=0.53$ ) and, overall, increasing fatigue across the three sessions. Ratings of fatigue during the Day Shift actually decreased very slightly across sessions, while ratings of fatigue during the Midshift increased across sessions.

Fear. There were no significant differences found for any main effect or interaction for self-reported level of fear (see Figure 47).

Response Time. Analysis of the response time for registering responses to the mood scale items yielded two significant interactions, Drug $\mathrm{x}$ Shift, $F(1,15)=$ 5.16, $p=.04$, and Shift $\times$ Session, $F(2,30)=4.50, p$ $=.02$ (see Figure 48). Subjects responded fastest under the placebo condition during the Day Shift and slowest under the antihistamine condition during the Day Shift. The response speed of subjects during the Midshift under either antihistamine or placebo was very similar and about midway between the fastest and slowest response speed. Ignoring drug condition, subjects appeared to get slower in responding to the mood scale across the sessions in the Midshift condition but not so during the Day Shift.

\section{Self-Reported Workload Level - NASA TLX Ratings}

ATST NASA-TLX Ratings. Analysis of the NASA TLX workload assessment scale resulted in very little in the way of significant results. No significant differences were found for the Mental Demand, Physical
Demand, Temporal Demand, Performance, and Effort scales. The analysis of the Frustration scale yielded one significant finding, the Drug $\mathrm{x}$ Session interaction, $F(2,30)=3.45, p=.04$, which was largely due to an elevated frustration rating for the antihistamine group during the second session only.

MATB NASA-TLX Ratings. Workload ratings for the MATB task did yield more significant findings than those for the ATST task. A significant Drug main effect was found for the degree of Mental Demand, $F(1,15)=5.37, p=.04$. Subjects rated the MATB more mentally challenging during the antihistamine condition (antihistamine, $M=61.0, S D=17.6$; placebo, $M=58.6, S D=19.3$ ). The most consistent finding was a Shift $x$ Session interaction for Mental Demand, $F(2,30)=7.22, p=.003$, Physical Demand, $F(2,30)=8.55, p=.001$, Performance, $F(2$, $30)=4.91, p=.01$, and Frustration, $F(2,30)=5.55$, $p=.009$. In most of these cases, subjects rated the MATB as generally decreasing in demand across sessions during the Day Shift, and generally increasing in demand across sessions during the Midshift.

\subsection{CONCLUSIONS}

The purpose of this study was to investigate the effects of work shift (Day Shift vs. Midshift) and a specific antihistamine drug (chlorpheniramine maleate) on human operator performance. In addition, the effect of time on task, or successive drug doses, through the shift (Session effect) was also assessed. A battery of cognitive and human performance tasks was used to investigate these effects, as well as various self-report measures of physical state, mood, and workload.

The results of this study can best be summarized by Table 4, which provides an overview of the main effects and two-way interactions from the various analyses of variance. (There were no three-way interactions that were statistically significant.) What is immediately apparent from Table 4 is that the greatest number of significant findings existed for the Drug main effect and the Shift $x$ Session interaction. The Shift and Session main effects evidenced considerably fewer significant findings, and the other two interactions had very few significant findings. Of course, the simple frequency or even magnitude of significant effects may not be the best way in which to evaluate the outcome of an investigation. However, in this case, the general trends in significant findings were not only apparent: but were also consistent with prior research evidence. 
Because this study attempted to explore factors that may affect safety-sensitive job performance, a broad range of tasks and self-report measures was used and a somewhat conservative examination of the results was conducted. This conservative approach possibly resulted in an over-inclusiveness with regard to significant findings. Thus, an examination of general trends in the findings is an important consideration in this study. Evidence of a factor having an influence across the performance and self-report measures, especially if that influence is consistently negative, would raise much more concern regarding that factor than would a random pattern of significant findings. Such a case of consistent negative influence existed for two factors in this study.

The administration of chlorpheniramine maleate appeared to have fairly profound negative effects across a wide range of performance measures. Statistically significant negative effects resulted for some component of every simple, dual, and complex task that subjects performed. This trend in the results suggested that, in general, the effects of chlorpheniramine maleate were not selective across various types of tasks. This finding was in agreement with several past studies that found a variety of negative cognitive and psychomotor effects due to chlorpheniramine maleate (Chapman \& Rawlins, 1982; Clarke \& Nicholson, 1978; Higgins et al., 1968; Khosla et al., 1993; Lee et al., 1988; Nicholson et al., 1991; Witek et al., 1995). Such broad-ranging effects might also support the view that the actions of this drug disrupt performance resources at a more fundamental and broad level, such as basic central nervous system structures (see Lee et al., 1988, and Loring \& Meador, 1989), as opposed to a higher order or more selective level.

Despite the broad ranging effects of this drug, it was possible to identify more specific aspects of performance that were disrupted by its administration. For example, chlorpheniramine maleate exposure led to degraded tracking performance on every task that had a tracking component (Critical Tracking, both variations of the Dual Task, and the MATB). This result was consistent with the early work of Higgins et al. (1968) and Clarke and Nicholson (1978). In these cases, the most critical measure of tracking performance (RMS error, control losses, or mean lambda) showed poorer performance after chlorpheniramine maleate administration. Chlorpheniramine maleate exposure also led to degraded response speed in a wide range of tasks, a finding similar to those of Lee et al.
(1988) and Witek et al. (1995). While some of the simple tasks did not show RT differences (i.e., Spatial Processing), significantly slower response speed under chlorpheniramine maleate administration was found for five of the seven tasks that had response speed components. This result would suggest that chlorpheniramine maleate did have significant negative effects on the speed with which subjects responded to critical elements of these tasks. The negative effects on response speed of this drug were also recognized at another cognitive level. Subjects were not only significantly slowed in their processing of the performance tasks, they were also significantly slowed in their response to self-reported mood ratings. This would suggest that chlorpheniramine maleate not only caused slowing of the sensory motor components of task performance, but also caused slowing of the central processing of verbal tasks as well. This general slowing in performance is not surprising given the pharmacological action of chlorpheniramine maleate. As a CNS $\mathrm{H}_{1}$ blocker, chlorpheniramine maleate acts to reduce CNS activity and typically to lower general arousal. It should be noted that other researchers have failed to find such response time effects (Kulshrestha et al., 1978; Philpot et al., 1993), although methodological or sampling differences may explain this disparity.

While it is clear that chlorpheniramine maleate had a negative effect on a broad range of tasks and that it negatively affected the speed with which subjects responded to many tasks, it is less clear what effect chlorpheniramine maleate had on accuracy. Of the several tasks that provided accuracy measures, only two yielded significant Drug main effects for percent correct measures. Of interest though is the fact that the two tasks yielding significant effects for accuracy were both spatial tasks in nature. It is unclear whether chlorpheniramine maleate may exert negative effects on the accuracy of tasks related to spatial ability because of its pharmacological actions on neurological centers that bear on spatial perception, or whether the effects on accuracy are more broad-ranging, but simply not pronounced enough in this study given the dosages and tasks used. The latter might have been the case, because there was a significant difference for the accuracy measure for the Memory Task component of the Dual Task (Group Lambda). Another task that is often viewed as a spatial task (the Manikin Task component of the Attention Switching Task) did not evidence a significant difference in accuracy. The lack 
of a significant effect on accuracy for this task may be due to the fact that the Manikin Task has only eight possible orientations, and repeated performance of the task may lead to its becoming more of a pattern recognition task and less a task of spatial ability. Thus, the accuracy results are unclear, but the findings regarding spatial processing seem very much worth additional study, especially because this is such a critical skill for both ATCs and pilots.

The other area where many significant effects were found was the Shift $x$ Session interaction. This refers to the complex changes that occur across the three testing sessions for the Day Shift vs. the Midshift testing conditions. Once again, some component of every task showed a Shift $x$ Session interaction. In general terms, the form of these interactions suggested that performance for the Midshift was better than for the Day Shift during the initial session of the work shift (Session 1), but was worse than the Day Shift for the final session (Session 3). In many cases, this was a fairly complete cross-over interaction with the shift groups appearing to differ at Session 1 and then reversing the direction of their differences by Session 3. In other cases, the performance was different between the shift groups only at the beginning or at the end of the shift. In these cases, the differences were in the same direction as the differences in the cross-over interactions. For example, in nearly all cases where performance was comparable at the beginning of the shift, performance for the Day Shift was better at the end of the shift, and in nearly all cases where performance was comparable at the end of the shift, performance during the first session was better for the Midshift.

Viewed from a more general perspective, these results revealed that across the sessions, performance during the Day Shift generally got better and performance during the Midshift generally got worse. From this perspective, these results support past research that found sleep disruption or sleep loss degrade performance (Akerstedt, 1990; Collins, 1977; Monk, 1990; Naitoh, 1976; Taub \& Berger, 1973, 1976; Wilkinson, 1965).

At first, these results from the various Shift $x$ Session interactions appear confusing. There is commonalty among these findings, however. In comparative terms, Midshift performance seemed to be better or at least equal to Day Shift performance at the beginning of the shift, and then seemed to deteriorate as the shift continued. Performance during the Day Shift seemed equal to or worse than Midshift performance at the beginning of the shift, but then improved over the shift to end up being much better or at least equal to Midshift performance at the end of the shift. The nature of this relationship is complex, but consistent. Fortunarely, other measures in this study provided additional insight into this complex finding.

From the results of the Activity State Questionnaire, it was clear that subjects reported significantly more physical symptoms during the Midshift, as compared to the Day Shift, and they reported increasing levels of physical symptoms across the testing sessions. It should be noted that, in general, these are considered negative physical symptoms, and chlorpheniramine maleate administration did not play an important role in this effect. It appears that either the Session factor has a quite different effect on the sense of physical well being of the subjects, given the shift they are working, or there might be a circadian cycle of changes in physical symptoms in the opposite direction across the two shifts. The level of negative physical symptoms declined slowly across the Day Shift. Across the Midshift, the level of negative physical symptoms increased markedly. What is interesting is that the level of physical symptoms reported by subjects at the end of the Day Shift is quite similar to the level reported by subjects when they begin the Midshift. This is typically the lowest level of the day. In fact, these two points in time represent the same time of day.

Thus, while time on shift (or the Session effect) seems to improve the level of negative physical symptoms during the Day Shift, time on shift during the Midshift may simply reflect a growing decline in resources and adjustive capacity following a day of activity. In this regard, it is important to remember that this was not a study of sleep loss effects. Subjects in this study knew, much like they would know if they were actually working a job during the midshift time period, that they were going to be up through the night. Not unlike those who work midshift jobs, these subjects probably involved themselves in considerable daytime activity before reporting to the lab for testing. Thus, it is probably accurate to suspect that the Midshift is a shift worked after a period of daily activity, while the Day Shift is often followed by a period of daily activity. This suspicion is also supported by past research on sleep cycles that confirmed that sleep typically preceded day work shifts and followed afternoon and night work shifts (Tepas, 1982). The cumulative effect of prior daily activity, 
combined with accumulated job fatigue across the shift, probably resulted in increased physical symptoms and degraded performance. This suspicion was further supported by the ratings of subjects regarding how prepared they were to work. Ratings across the three sessions during the Day Shift for the "Prepared" item on the questionnaire were essentially equal (with a slight peak during Session 2). Ratings across the Midshift revealed that subjects felt less and less prepared to perform their work as the shift continued. Overall, these results of self-report measures revealed substantial consistency with other studies reporting negative worker reaction to the Midshift (Melton \& Bartanowicz, 1986; Melton et al., 1973).

Other self-report measures provided additional findings of interest. The Mood Scale II results revealed that chlorpheniramine maleate administration resulted in feelings of being less active and less happy. These results are consistent with other studies that have found similar effects on self-report measures due to chlorpheniramine administration (Chapman \& Rawlins, 1982; Clarke \& Nicholson, 1978; Kulshrestha et al., 1978; Nicholson et al., 1991), but once again are not in agreement with other researchers that have failed to find such self-report effects (Kulshrestha et al., 1978; Philpot et al., 1993). The Shift factor seemed to have an even greater effect in that not only did subjects feel less active and less happy during the Midshift as compared to the Day Shift, but also they felt more depressed and fatigued. As noted previously, the size of some of these effects was not great in absolute terms. The Shift $\mathrm{x}$ Session interaction discussed above was also present for these self-reported mood measures and these mood results paralleled the findings noted for the Activity State Questionnaire-that is, Day Shift ratings suggested mood improvement across the shift, while Midshift ratings suggested mood decline across the shift. This pattern of mood change is quite consistent with the patterns of mood change across shift found by Schroeder, Rosa, Witt, and Banks (1995).

Self-report measures of workload were not as effective in revealing significant differences as were other self-report measures, but the pattern of self-reported workload differences was interesting. The NASA-TLX measure yielded essentially no differences due to chlorpheniramine maleate administration. This would suggest that the NASA-TLX was relatively insensitive to the antihistamine effect. This finding was not necessarily surprising, because the NASA-TLX was not necessarily designed as a measure of stressor effects. It is possible that subjective task difficulty can vary as a result of changing objective task parameters or by changing the state of the person performing the task (by introducing drugs that would increase or decrease performance capability, for example). However, only when state factors, such as drug effects or fatigue, reach levels that require considerable compensatory effort (and thereby obscure the difference between increased task difficulty and degraded personal capability) would subjects rate workload as higher. Chlorpheniramine maleate exposure alone was only powerful enough to make a modest change in NASA TLX ratings, as evidenced by the increased Mental Effort for the MATB under the drug condition. Assuming that this finding was not just Type I error, it is in agreement with other findings that suggest chlorpheniramine maleate leads to greater difficulty in concentration (Khosla et al., 1993; Lee et al., 1988). On the other hand, there were several self-reported differences in workload for the Shift $x$ Session interaction. Again, the ratings of various aspects of workload were much like the ratings for mood and physical condition. Subjects generally felt that workload decreased across the Day Shift, but seemed to increase across the Midshift. In this situation, the combined influence of these factors may have been enough to cause subjects to subjectively view the workload as having changed.

The fact that there were significant differences in subjective workload ratings for the MATB and not for the ATST is also not necessarily surprising. The ATST is a more intrinsically motivating task than the MATB, it does not have the same degree of pressure to perform at increasingly higher levels as the MATB, and it was not performed for as long as the MATB. For these reasons, the MATB was a more demanding task overall. This higher level of demand may have increased the possibility that workload differences were more recognizable for the MATB, as compared to the ATST.

The interpretation of the remaining significant findings for the Shift and Session main effects are difficult. These significant findings are clearly far fewer in number and are further clouded by the fact that the Shift $\mathrm{x}$ Session interaction was so influential. Prudence would suggest that these results be cautiously generalized. In all cases, however, these effects seemed to parallel the general findings already seen for the Drug and Shift x Session factors. 
Finally, there are a few additional observations that are noteworthy. First, there did not seem to be strong evidence for a pronounced negative effect as the result of combining antihistamine and shift, as Higgins et al. (1968) found with chlorpheniramine maleate and altitude. This was surprising because logic would suggest that a sedating drug in combination with a fatiguing work shift might lead to an even more dramatic negative effect on performance than either factor alone or than their simple additive effect. This did not appear to be the case in this study. Prudence, and perhaps simple skepticism, would suggest that this finding be further explored.

Second, the value of assessing change within a shift was self-evident in this study. The dynamics of performance and mood change across shifts were quite different, and the presence of cross-over interactions would suggest that conducting studies without repeated measures across shifts (i.e., using global measures summed across the entire shift) would obscure these very dramatic differences.

Third, asking subjects about antihistamine effects based on known side-effects does not seem like a very productive way to assess their influence, at least in the manner used in this study. Perhaps a more sensitive response scale might gain better results. The measure used in this study was clearly not effective for the dose and level of side-effects experienced.

And fourth, there was some limited evidence of differential sensitivity within tasks for various risk factors. For example, the Attention Switching Task provided some interesting results in addition to those already noted. This task directed the subject to perform either the Manikin Task or Mathematical Processing Task component. The Mathematical Processing Task appeared to be reasonably sensitive to the effects of chlorpheniramine maleate, while the Manikin Task appeared to be sensitive to the combined effects of Shift and Session. The Critical Tracking Task provided another possible example of differential sensitivity, and perhaps even the Spatial Processing Task. This apparent differential sensitivity, while intriguing, must be interpreted cautiously. It may be that some of these tasks do offer dependent measures that respond differentially to various risk factors. It may also be the case that other factors may have simply reduced the possibility of finding significant differences for that measure in relationship to other factors in this study.
In summary, the findings of this study suggest that chlorpheniramine maleate alone has a fairly strong negative influence on a wide range of task performance and mood measures. There also seems to be a rather complex relationship between work shift and time on the shift (Session) such that performance and mood during the Day Shift tend to get better and during the Midshift tend to get worse. No evidence was found that chlorpheniramine maleate and work shift combine in producing a multiplicative effect on performance or mood.

\section{REFERENCES}

Akerstedt, T. (1990). Psychological and psychophysiological effects of shift work. Scandinavian Journal of Work, Environment, and Health, 16, 67-73.

Alluisi, E.A., and Morgan, B.B. (1982). Temporal factors in human performance and productivity. In E.A. Alluisi and E.A. Fleishman (eds.) Human performance and productivity, Vol. 3: Stress and performance effectiveness. Hillsdale, NJ: Lawrence Earlbaum Associates.

Aschoff, J. (1965). Circadian rhythms in man. Science, $148,1427-32$.

Aschoff, J. (1981). Circadian rhythms: Interference with the dependence on work-rest schedules. In L.C. Johnson, D.I. Tepas, W.P. Colquhoun, and M.J. Colligan (eds.) Advances in sleep research, Vol. 7: Biological rhythms, sleep, and shift work. New York, NY: Spectrum Publications Medical \& Scientific Books.

Betts, T., Markman, D., Debenham, S., Mortiboy, D., and McKevitt, T. (1984). Effects of two antihistamine drugs on actual driving performance. British Medical Journal, 288, 281-2.

Bovet, D., Horclois, R., and Walthert, F. (1944). Propriétés antihistaminiques de la $\mathrm{N}-p$ méthoxybenzyl-N-diméthyl-aminoéthyl $\alpha$ amino-pyridine. C. r. Séanc. Soc. Biol., 138, 99100. (cited in Goodman and Gilman, 1975).

Bovet, D., and Staub, A. (1937). Action protectrice des éthers phénoliques au cours de l'intoxication histaminique. Séanc. Soc. Biol., 124, 547-9. (Goodman and Gilman, 1975). 
Burns, M., Shanaman, J.E., and Shellenberger, C.H. (1994). A laboratory study of patients with chronic allergic rhinitis: Antihistamine effects on skilled performance. Journal of Allergy and Clinical Immunology, 93, 716-24.

Canfield, D., Flemig, J., and Hordinsky, J. (1995). Drugs and alcohol found in fatal civil aviation accidents between 1989 and 1993. (DOT/FAA/ AM-95/28). Washington, DC: Federal Aviation Administration, Office of Aviation Medicine. (N'TIS \# ADA302 527).

Chapman, R.H., and Rawlins, M.D. (1982). A randomized single-blind study of astemizole and chlorpheniramine in normal volunteers. British Journal of Clinical Pharmacology, 13, 593.

Cingi, E., Cingi, I.M., and Cingi, C. (1990). Effects of antihistamines on psychomotor performance. European Journal of Pharmacology, 183, 535.

Clarke, C.H., and Nicholson, A.N. (1978). Performance studies with antihistamines. British Journal of Clinical Pharmacology, 6, 31-5.

Collins, W.E. (1977). Some effects of sleep deprivation on tracking performance in static and dynamic environments. Journal of Applied Psychology, 62, 567-73.

Colquhoun, W.P. (1982). Biological rhythms and performance. In W. Webb (ed.) Biological rhythms, sleep, and performance. New York, NY: Wiley.

Cruz, C.E., and Della Rocco, P.S. (1995). Sleep patterns in air traffic controllers working rapidly rotating shifts: $A$ field study. (DOT/FAA/AM-95/ 12). Washington, DC: Federal Aviation Administration, Office of Aviation Medicine. (NTIS \# ADA294 159/9GAR).

Della Rocco, P.S., and Cruz, C.E. (1995). Shift work, age, and performance: Investigation of the 2-2-1 shift schedule used in air traffic control facilities: 1 . The sleep/wake cycle. (DOT/FAA/AM-95/19). Washington, DC: Federal Aviation Administration, Office of Aviation Medicine. (NTIS \# ADA295 542/5INT).

Eddy, D.R., Dalrymple, M.A., and Schiflett, S.G. (1992). Comparative effects of antihistamines on aircrew mission effectiveness under sustained operations. (AL-TR-1992-0018). Brooks AFB, TX: USAF Armstrong Laboratory, Crew Systems Directorate. (NTIS \# ADA254 203/3).
Fink, M., and Irwin, P. (1979). CNS effects of the antihistamines diphenhydramine and terfenadine. Pharmacopsychiatry, 12, 35-44.

Folkard, S. (1981). Shiftwork and performance. In L.C. Johnson, D.I. Tepas, W.P. Colquhoun, and M.J. Colligan (eds.) Advances in sleep research, Vol. 7: Biological rhythms, sleep, and shift work. New York, NY: Spectrum Publications Medical \& Scientific Books.

Folkard, S., and Monk, T.H. (1985). Circadian performance rhythms. In S. Folkard and T.H. Monk (eds.) Hours of work: Temporal factors in work scheduling. New York, NY: Wiley.

Gilliland, K., and Schlegel, R.E. (1992). Evaluation of extended practice effects on the Air Traffic Scenarios Test. (Final Report on Contract DTFA-0292P13359). Oklahoma City, OK: Federal Aviation Administration, Civil Aeromedical Institute.

Gilliland, K., and Schlegel, R.E. (1993). Readiness-toperform testing: A critical analysis of the concept and current practices. (DOT/FAA/AM-93/13). Washington, DC: Federal Aviation Administration, Office of Aviation Medicine.

Gilliland, K., and Schlegel, R.E. (1995, January). Readiness-to-perform testing and the worker. Ergonomics in Design, 3, 14-9.

Gilliland, K., and Schlegel, R.E. (1997). Laboratory model of readiness-to-perform testing, Volume I: Learning rates and reliability analyses for candidate readiness-to-perform measures. (DOT/FAA/AM97/5). Washington, DC: Federal Aviation Administration, Office of Aviation Medicine. (NTIS \# ADA323 620/5INZ).

Goodman, L.S., and Gilman, A. (1975). The pharmacological basis of therapeutics, 5th ed. New York, NY: Macmillan.

Goodman, L.S., and Gilman, A. (1990). The pharmacological basis of therapeutics, 18th ed. New York, NY: Pergamon Press.

Higgins, E.A., Davis, A.W., Fiorica, V., Iampietro, P.F., Vaughan, J.A., and Funkhouser, G.E. (1968). Effects of two antihistamine-containing compounds upon performance at three altitudes. (AM-68-15). Washington, DC: Federal Aviation Administration, Office of Aviation Medicine. (NTIS \# AD 675 502). 
Hindmarch, I., Kerr, J.S., and Sherwood, N. (1991). The effects of alcohol and other drugs on psychomotor performance and cognitive function. Alcohol and Alcoholism, 26, 71-9.

Johnson, R.F., and McMenemy, D.J. (1989). Antihistamines and sentry duty: Effects of terfenadine and diphenhydramine on target detection and rifle marksmanship. Fort Detrick, MD: U.S. Army Medical Research and Development Command. (NTIS \# ADA209 754/1).

Khosla, P.P., Saha, N., Koul, A., Chakrabarti, A., Sankaranarayanan, A., and Sharma, P.L. (1993). Effects of ranitidine alone and in combination with chlorpheniramine on histamine-induced wheal and flare and psychomotor performance. Indian Journal of Physiology and Pharmacology, $37,132-4$.

Kleitman, N. (1963). Sleep and wakefulness. Chicago, IL: University of Chicago Press.

Kulshrestha, V.K., Gupta, P.P., Turner, P., and Wadsworth, J. (1978). Some clinical pharmacological studies with terfenadine, a new antihistamine drug. British Journal of Clinical Pharmacology, 6, 25-9.

Lee, A., Lader, M., and Kitler, M.E. (1988). The psychopharmacological effects of single doses of prolonged release formulations of dimethindene and chlorpheniramine in human volunteers. $\mathrm{Hu}$ man Psychopharmacology, 3, 111-7.

Loring, D.W., and Meador, K.J. (1989). Central nervous system effects of antihistamines on evoked potentials. Annals of Allergy, 63, 604-7.

Manning, C., and Gengo, F.M. (1993). Effects of drugs on human functioning: Antihistamines. Progress in Basic Clinical Pharmacology, 9, 52-69.

Meador, K.J., and Loring, D.W. (1988). Differential cognitive effects of terfenadine and chlorpheniramine. Journal of Allergy and Clinical Immunology, 81, 177 (abstract).

Melton, C.E., and Bartanowicz, R.S. (1986). Biological rhythms and rotating shift work: Some considerations for air traffic controllers and managers. (DOT/FAA/AM/86-2). Washington, DC: Federal Aviation Adminsitration, Office of Aviation Medicine. (NTIS \# ADA168 742/5).
Melton, C.E., McKenzie, J.M., Smith, R.C., Polis, B.D., Higgins, E.A., Hoffman, S.M., Funkhouser, G.E, and Saldivar, J.T. (1973). Physiological, biochemical, and psychological responses in air traffic control personnel: Comparison of the 5-day and 2-2-1 shiff rotation patterns. (FAA-AM-73-22). Washing ton, DC: Federal Aviation Administration, Office of Aviation Medicine. (NTIS \# AD 778 214/7GI)

Melton, C.E., Smith, R.C., McKenzie, J.M., Saldivat J.T., Hoffman, S.M., and Fowler, P.R. (1975) Stress in air traffic controllers: Comparison of two air route traffic control centers on different shiff rotation patterns. (FAA-AM-75-7). Washington DC: Federal Aviation Administration, Office of Aviation Medicine. (NTIS \# ADA020 679/7Gl)

Meltzer, E.O. (1990). Performance effects of antihis tamines. Journal of Allergy and Clinical Immuno ogy, 86, 613-9.

Meltzer, E.O. (1991). Comparative safety of $\mathrm{H}_{1}$ an histamines. Annals of Allergy, 67, 625-33.

Monk, T.H. (1990). Shiftworker performance. In A Scott (ed.) Occupational medicine: State of the reviews. Philadelphia, PA: Hanley and Belfus:

Naitoh, P. (1976). Sleep deprivation in human sul jects: A reappraisal. Waking Sleeping, 1, 56-6

Nesthus, T.E., Schiflett, S.G., Eddy, D.R., a Whitmore, J.N. (1991). Comparative effecti antihistamines on aircrew performance of sim. and complex tasks under sustained operations ( $A$ TR-91-104). Brooks AFB, TX: USAF Armstro Laboratory, Crew Technology Division. (Ni \# ADA248 752/8).

Nicholson, A.N. (1979). Effect of the antihistamir brompheniramine maleate and triprolidine drochloride, on performance in man. $B r$ Journal of Clinical Pharmacology, 8, 321-4.

Nicholson, A.N. (1985). Central effects of $\mathrm{H}_{1}$ and antihistamines. Aviation, Space, and Envi, mental Medicine, 56, 293-8.

Nicholson, A.N., Pascoe, P.A., Turner, C., Gand C.R., Greengrass, P.M., Casy, A.F., and Me A.D. (1991). Sedation and histamine $H_{1}-r_{8}$ tor antagonism: Studies in man with the en? omers of chlorpheniramine and dimethind British Journal of Pharmacology, 104, 270-6 
Nicholson, A.N., Smith, P.A., and Spencer, M.B. (1982). Antihistamines and visual function: Studies on dynamic visual acuity and the pupillary response to light. British Journal of Clinical Pharmacology, 14, 683-90.

Nicholson, A.N., and Stone, B.M. (1982). Performance studies with the $\mathrm{H}_{1}$-antihistamine antagonists, astemizole and terfenadine. British Journal of Clinical Pharmacology, 13, 199-202.

Nicholson, A.N., and Stone, B.M. (1986). Antihistamines: Impaired performance and the tendency to sleep. European Journal of Clinical Pharmacology, 30, 27-32.

Pennebaker, J.W. (1982). The psychology of physical symptoms. New York, NY: Springer-Verlag.

Philpot, E.E., Biegalski, C.S., and Brooker, A.E. (1993). Effects of sedating and nonsedating antihistamines on flying performance. Military Medicine, 158, 654-60.

Prell, G.D., and Green, J.P. (1986). Histamine as a neuroregulator. Annual Review of Neuroscience, 9, 209-54.

Price, W., and Holley, D. (1990). Shift work and safety in aviation. In A.J. Scott (ed.) Occupational medicine: State of the art reviews, Vol. 5. Philadelphia, PA: Hanley and Belfus.

Reeves, D.L., Blackwell, E.A., Molina, E.A., and Hixson, W.C. (1989). New methodologies for assessing effects of pharmaceutical compounds on military performance. Pensacola, FL: Naval Aerospace Medical Research Laboratory. (NTIS \# ADA227-495).

Rice, V.J., and Snyder, H.L. (1993). The effects of Benadryl $^{\otimes}$ and Hismanal ${ }^{\otimes}$ on psychomotor performance and perceived performance. Aviation, Space, and Environmental Medicine, 64, 726-34.

Rosekind, M.R., Gander, P.H., Miller, D.L., Gregory, K.B., Smith, R.M., Weldon, K.J., Co, E.L., McNally, K.L., and Lebacqz, J.V. (1994). Fatigue in operational settings: Examples from the aviation environment. Human Factors, 36, 327-38.

Rutenfranz, J., Aschoff, J., and Mann, H. (1972). The effects of a cumulative sleep deficit, duration of preceding sleep period and body-temperature on multiple choice reaction time. In W.P. Colquhoun (ed.) Aspects of buman efficiencyDiurnal rhythm and loss of sleep. London: English Universities Press.
Saldivar, J., Hoffman, S., and Melton, C. (1977). Sleep in air traffic controllers. (DOT/FAA/AM-77-5). Washington, DC: Federal Aviation Administration, Office of Aviation Medicine. (NTIS \# ADA038 297/8GI).

Schlegel, R.E., and Gilliland, K. (1992). Development of the UTC-PAB normative database (AL-TR-920145). Wright-Patterson AFB, OH: USAF Armstrong Laboratory.

Schlegel, R.E., Shehab, R.L., and Gilliland, K (1994). Microgravity effects on cognitive performance measures: Practice schedules to acquire and maintain performance stability (AL-CF-TR-1994-0040). Brooks AFB, TX: USAF Armstrong Laboratory, Crew Technology Division.

Schroeder, D.J., and Goulden, D.R. (1983). A bibliography of shift work research: 1950-1982. (FAAAM-83-17). Washington, DC: Federal Aviation Administration, Office of Aviation Medicine. (NTIS \# ADA135 644/3).

Schroeder, D.J, Rosa, R.R., Witt, L.A., and Banks, B. (1995). Effects of 8-vs. 10-hour work schedules on the performancelalertness of air traffic control specialists. (DOT/FAA/AM-95/32). Washington, DC: Federal Aviation Administration, Office of Aviation Medicine. (NTIS \# ADA302 810/ 7INZ).

Snyder, H.L., and Berg, V.J. (1990). Complex cognitive performance and antibistamine use. (DAMD17-89-C-9073). Fort Detrick, MD: U.S. Army Medical Research and Development Command. (NTIS \# ADA224 602/3).

Stephens, R.L., Caldwell, J.A., Comperatore, C.A., Pearson, J.Y., and Delrie, D.M. (1992). Effects of terfenadine and diphenhydramine on brain activity and performance in a UH-60 flight simulator. Fort Rucker, AL: U.S. Army Aeromedical Research Laboratory. (NTIS \# ADA258 012/4).

Taub, J.M., and Berger, R.J. (1973). Performance and mood following variations in the length and timing of sleep. Psychophysiology, 10, 559-70.

Taub, J.M., and Berger, R.J. (1976). The effects of changing phase and duration of sleep. Journal of Experimental Psychology: Human Perception and Performance, 2, 30-41.

Tepas, D.I. (1982). Work/sleep time schedules and performance. In W. Webb (ed.) Biological rhythms, sleep, and performance. New York, NY: Wiley. 
Tharion, W.J., McMenemy, D.J., and Rauch, T.M. (1994). Antihistamine effects on the central nervous system, cognitive performance and subjective states. Neuropsychobiology, 29, 97-104.

Turek, F. (1986). Circadian principals and design of rotating shift work schedules. American Journal of Physiology, 251, R636-8.

Wever, R.A. (1979). The circadian system of man: Results of experiments under temporal isolation. New York, NY: Springer-Verlag.

Wever, R.A. (1981). On varying work-sleep schedules: The biological rhythm perspective. In L.C. Johnson, D.I. Tepas, W.P. Colquhoun, and M.J. Colligan (eds.) Advances in sleep research, Vol. 7: Biological rhythms, sleep, and shift work. New York, NY: Spectrum Publications Medical \& Scientific Books.
Wever, R.A. (1985). Man in temporal isolation: Bace principles of the circadian system. In S. Folkat and T.H. Monk (eds.) Hours of work: Tempor factors in work scheduling. New York, NY: Wild

Wilkinson, R.T. (1965). Sleep deprivation. In O.C Edholm and A. Bacharach (eds.) The physiology buman survival. New York, NY: Academic.

Williams, H.L., Lubin, A., and Goodnow, J.L. (1959 Impaired performance with acute sleep lo Psychological Monographs, 73, 1-26.

Witek, T.J., Canestrari, D.A., Miller, R.D., Yar J.Y., and Riker, D.K. (1995). Characterizati of daytime sleepiness and psychomotor perf mance following $\mathrm{H}_{1}$ receptor antagonists. Am of Allergy, Asthma, and Immunology, 74, 419-7 


\section{FIGURES}

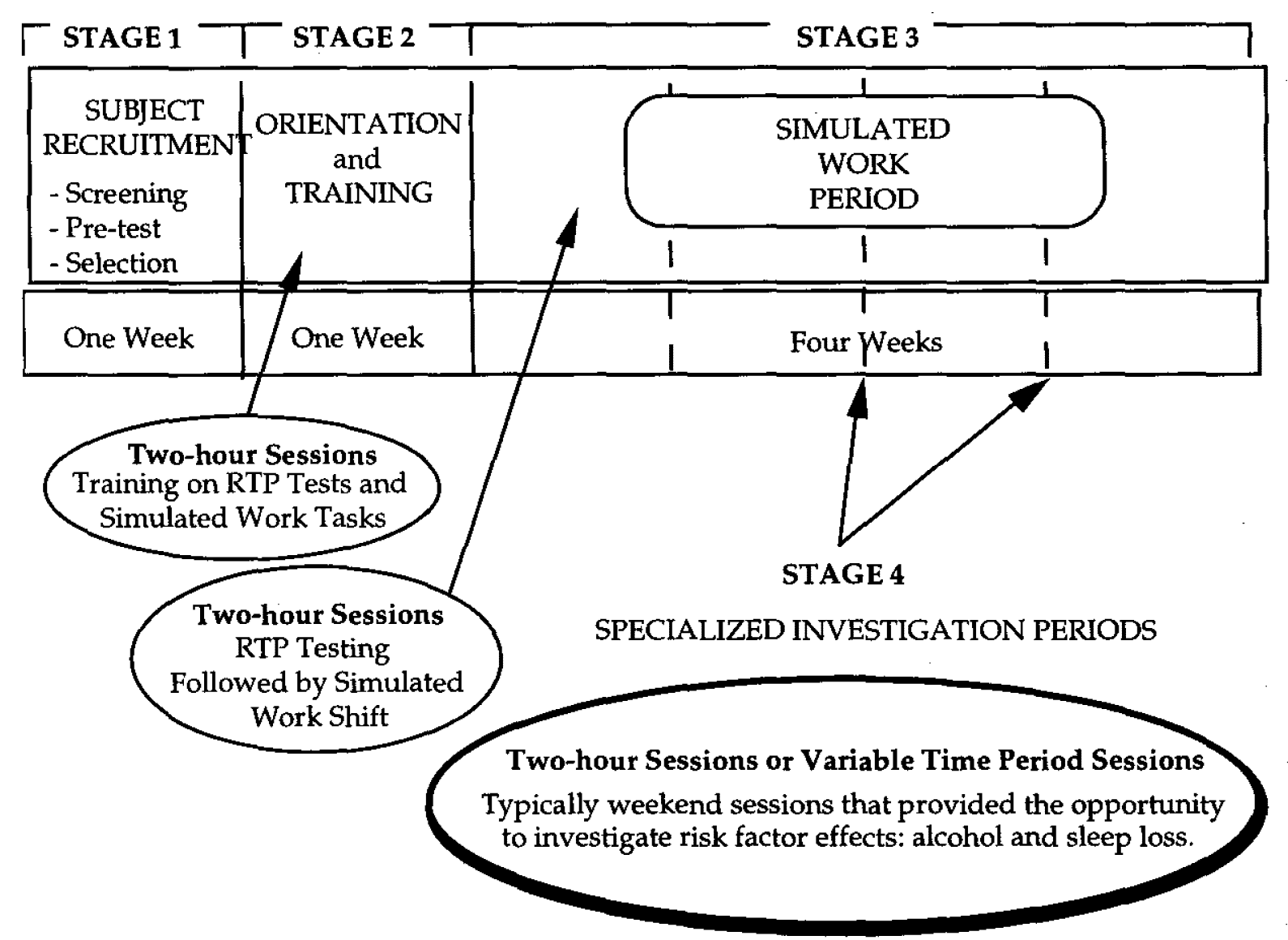

Figure 1. Laboratory Model of Readiness-to-Perform Testing. 


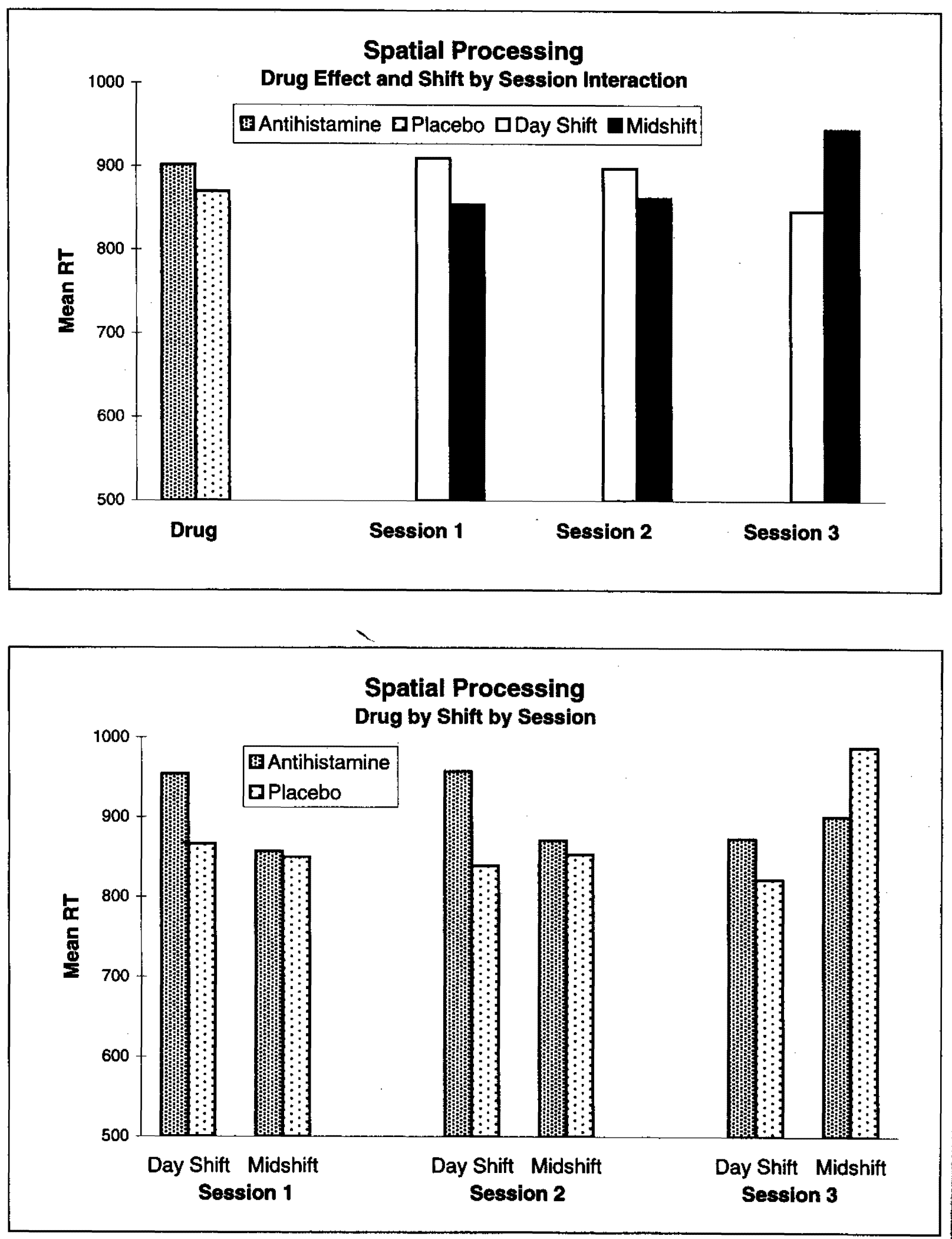

Figure 2. Spatial Processing Mean RT. 

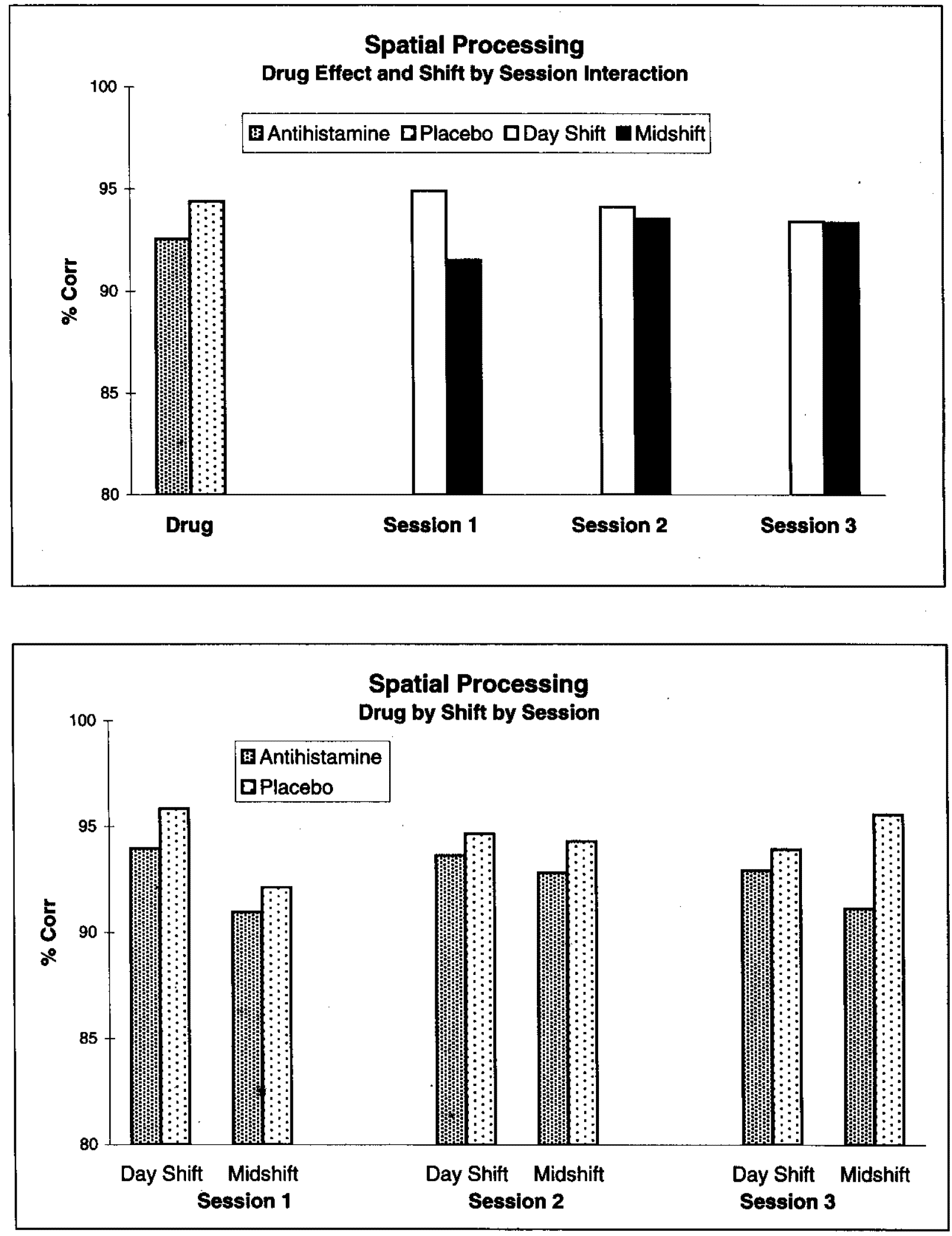

Figure 3. Spatial Processing Percent Correct. 


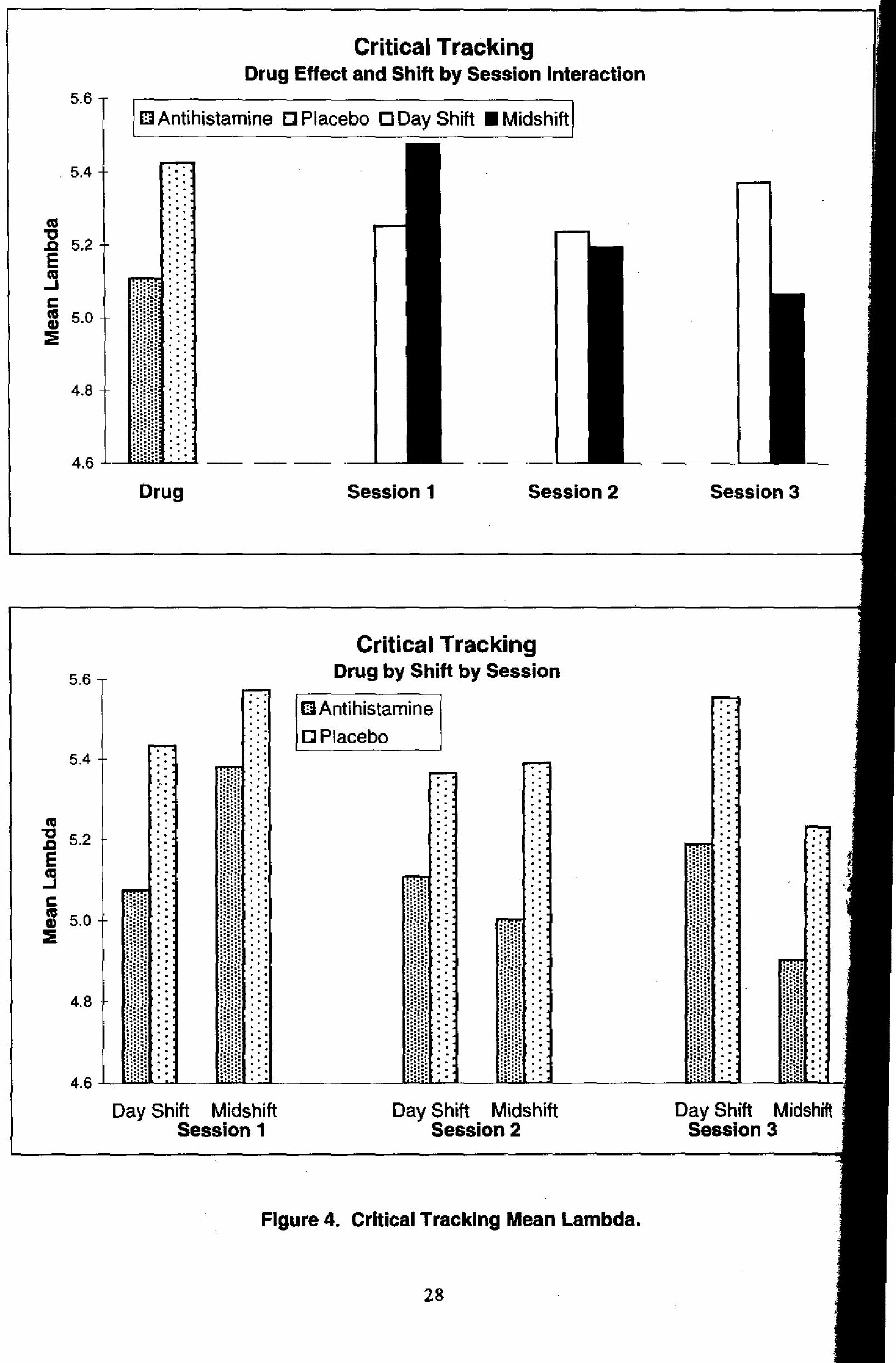



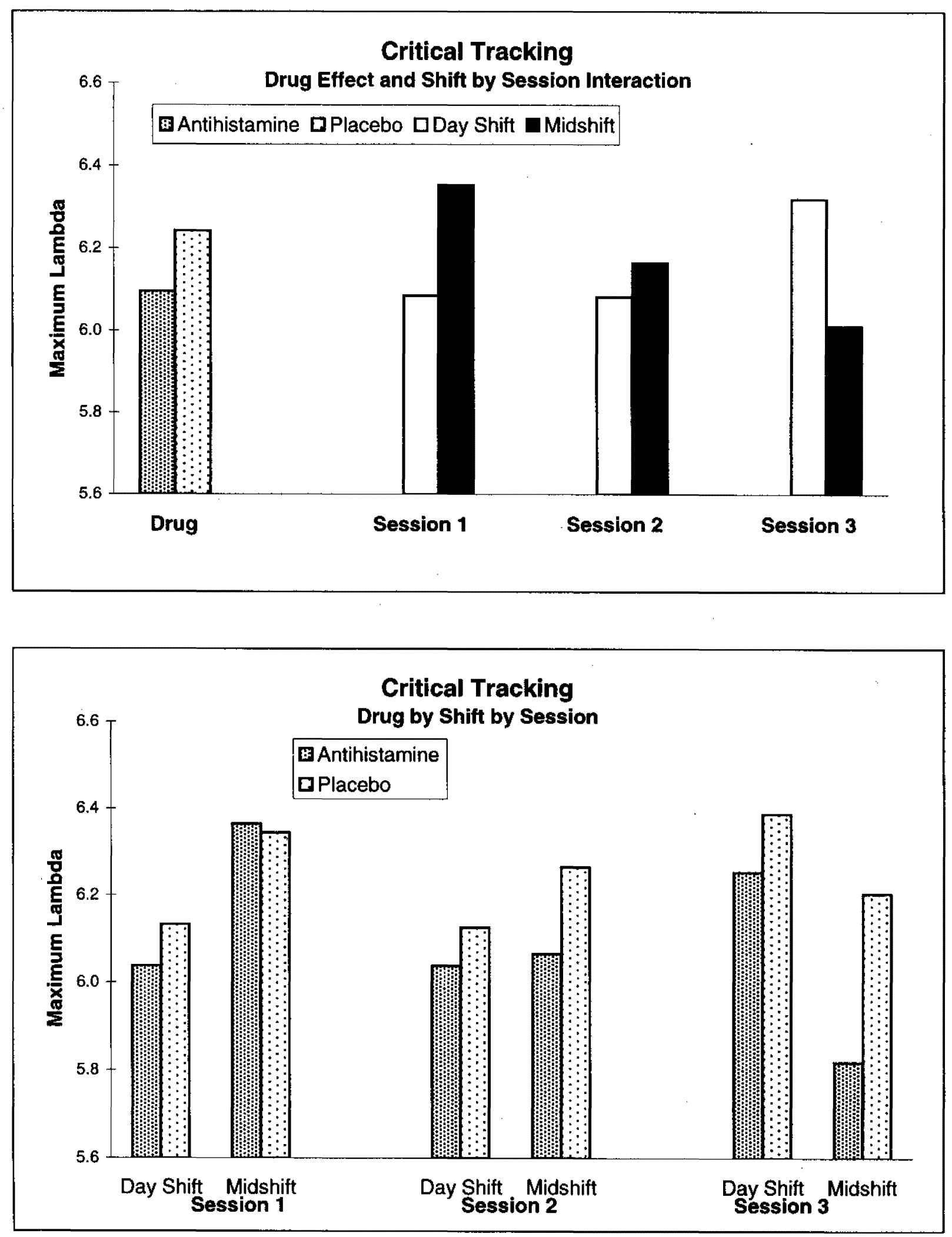

Figure 5. Critical Tracking Maximum Lambda. 

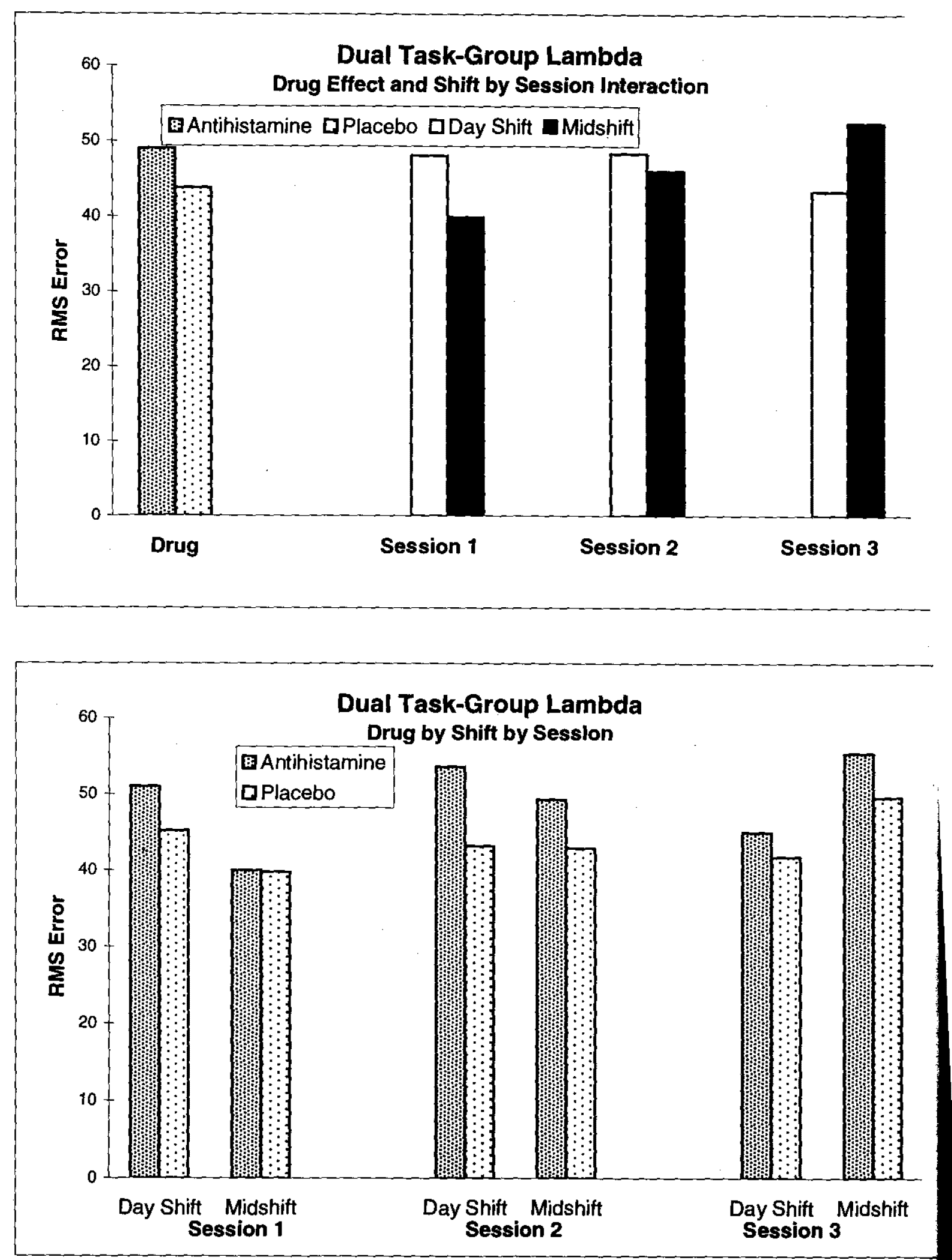

Figure 6. Dual Tracking-Group Lambda RMS Error. 

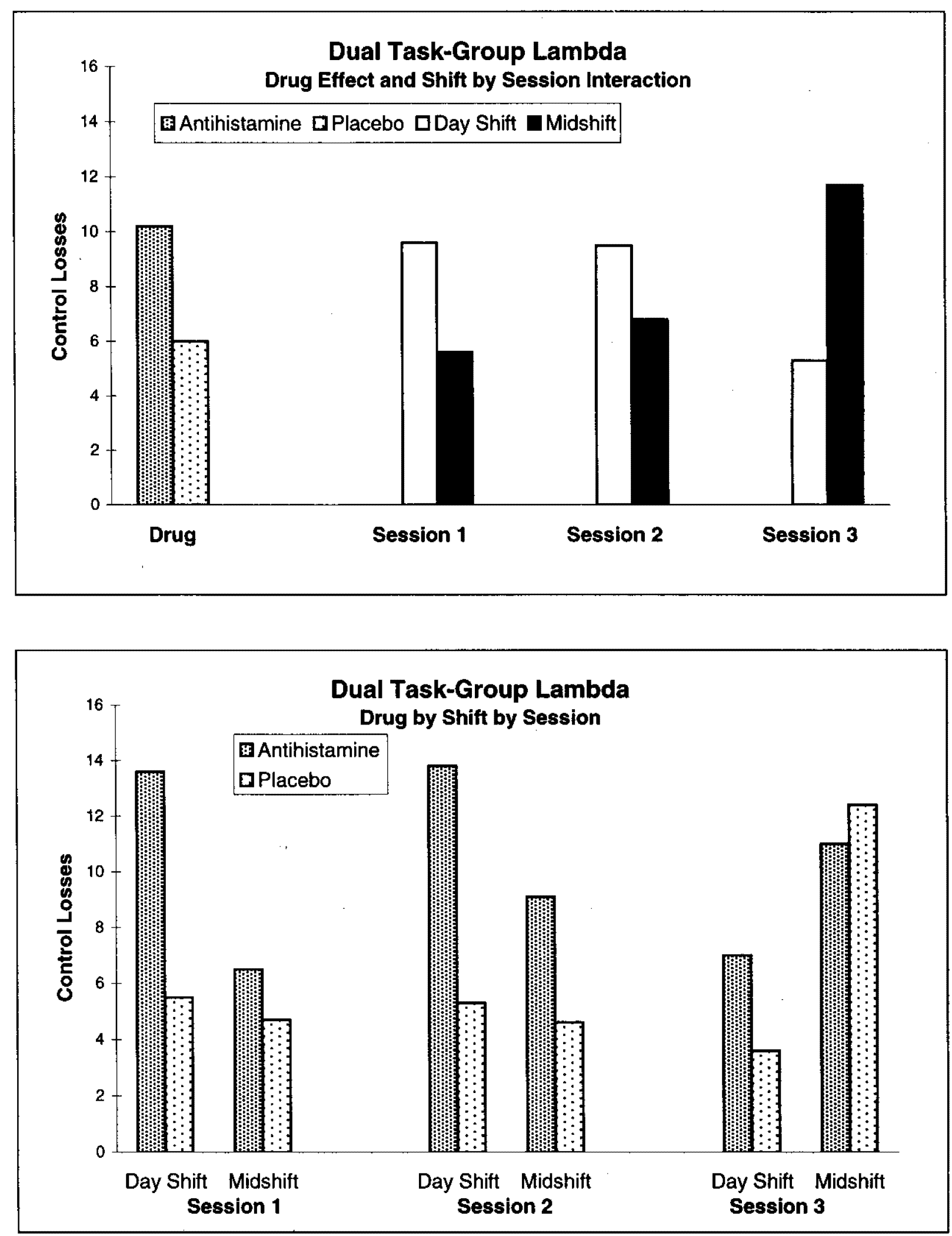

Figure 7. Dual Tracking-Group Lambda Control Losses. 

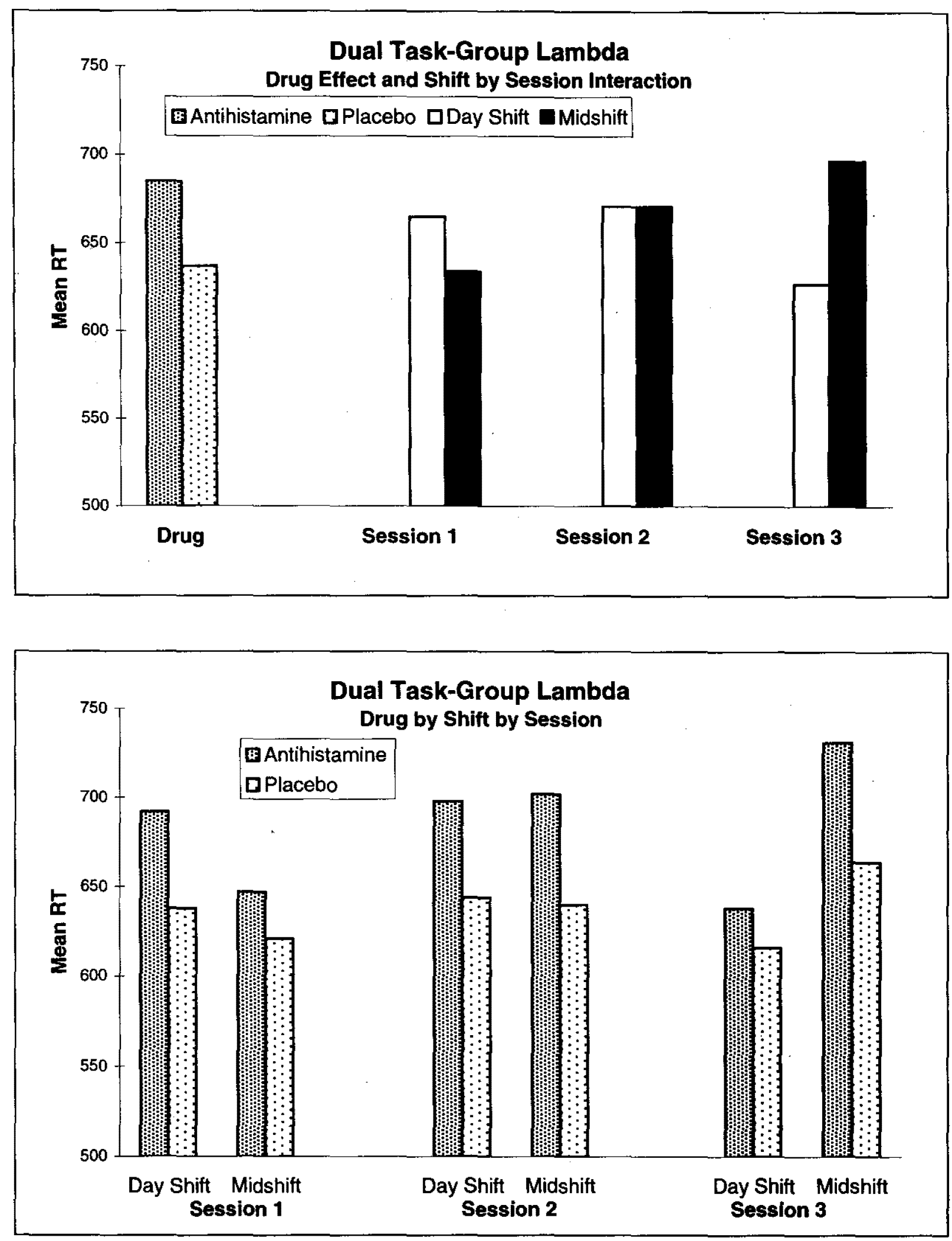

Figure 8. Dual Memory Search-Group Lambda Mean RT. 

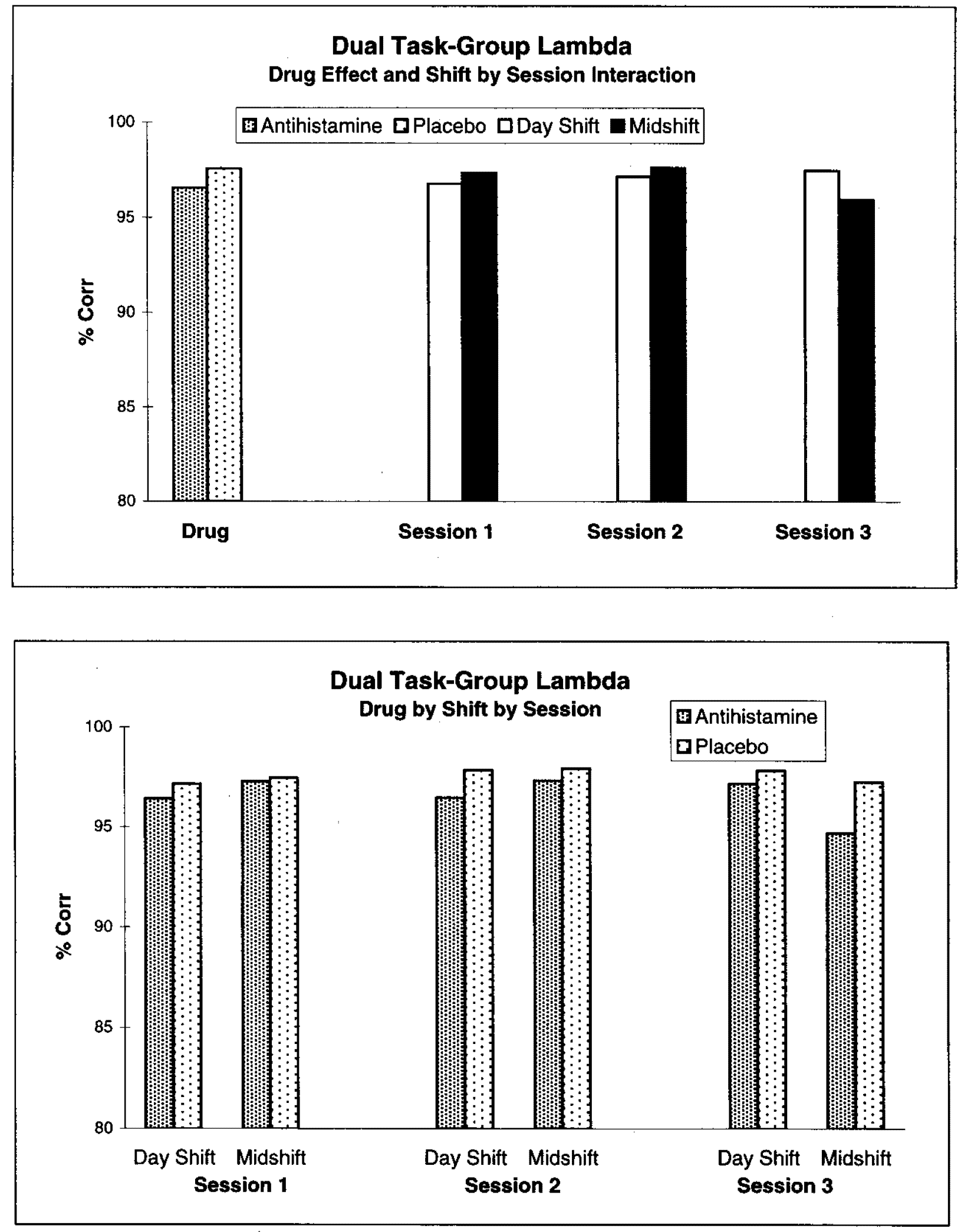

Figure 9. Dual Memory Search-Group Lambda Percent Correct. 

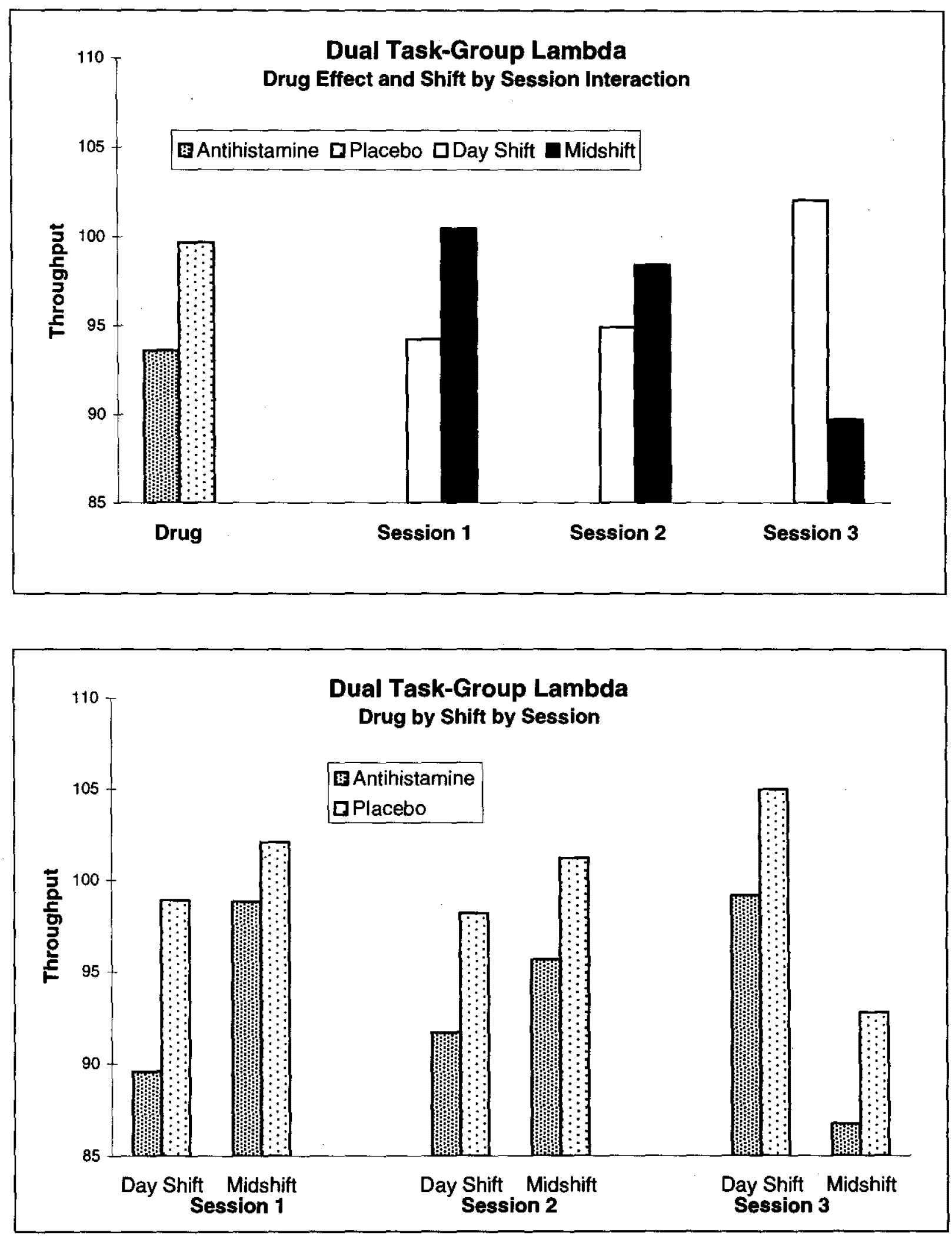

Figure 10. Dual Memory Search-Group Lambda Throughput. 

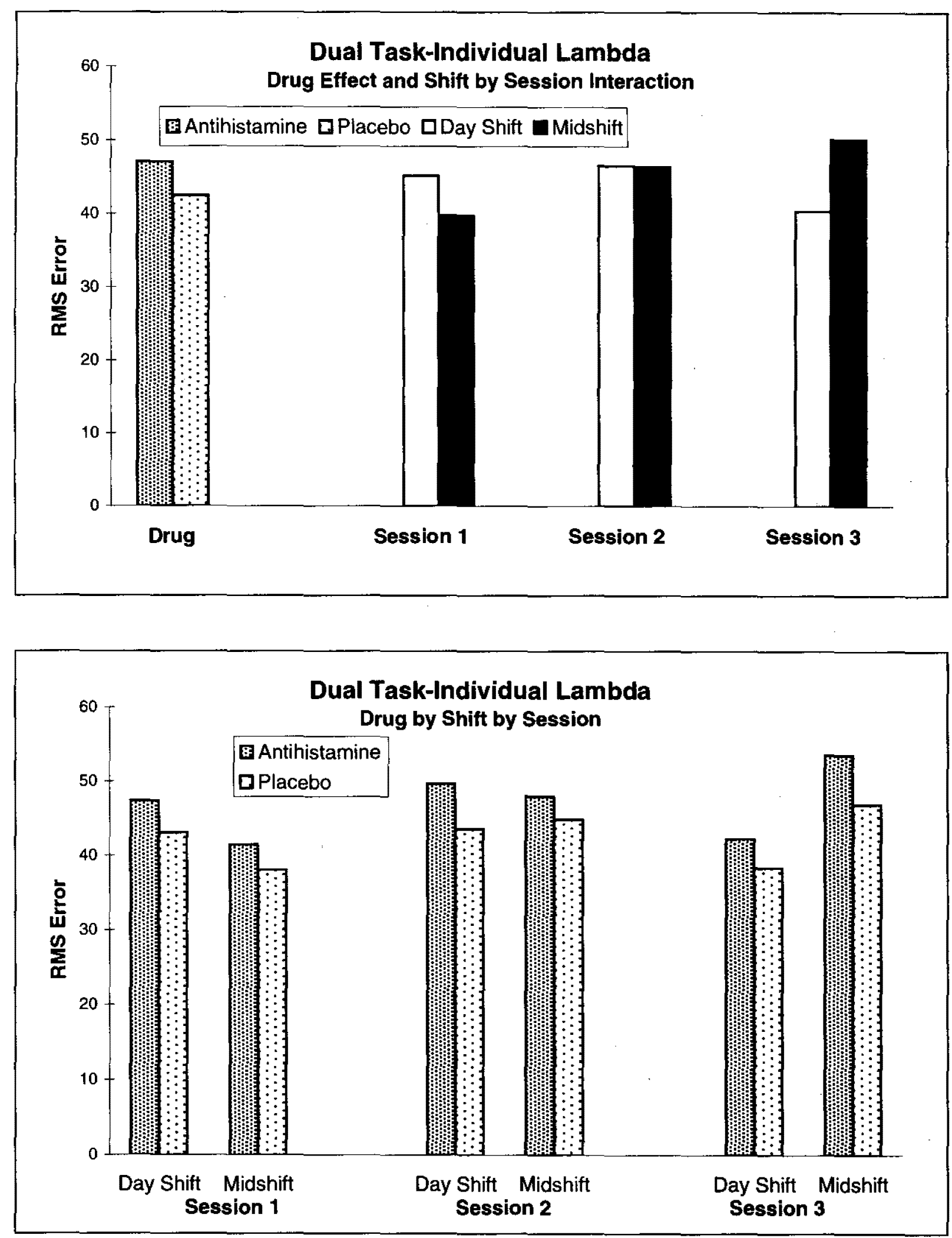

Figure 11. Dual Tracking-Individual Lambda RMS Error. 

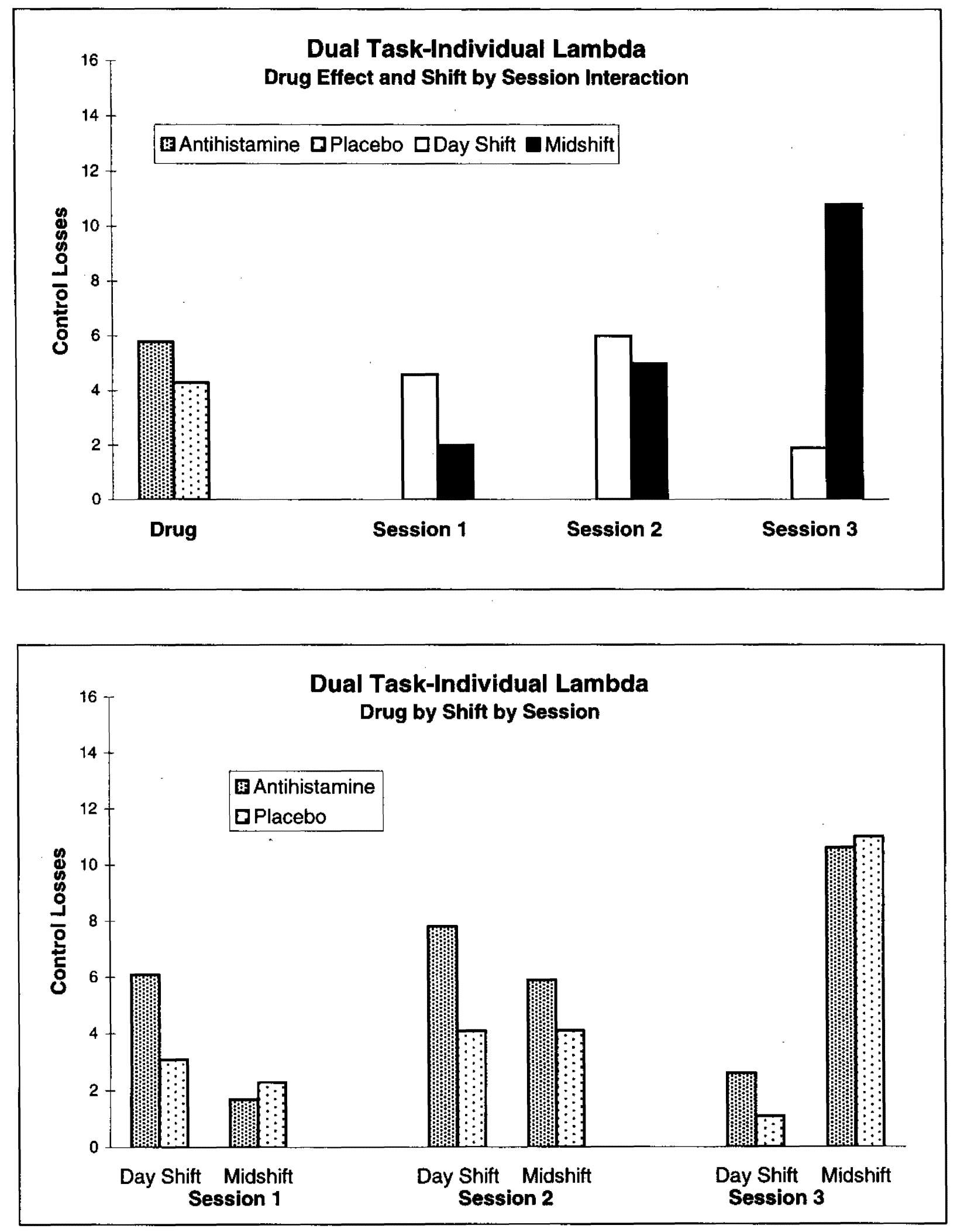

Figure 12. Dual Tracking-Individual Lambda Control Losses. 

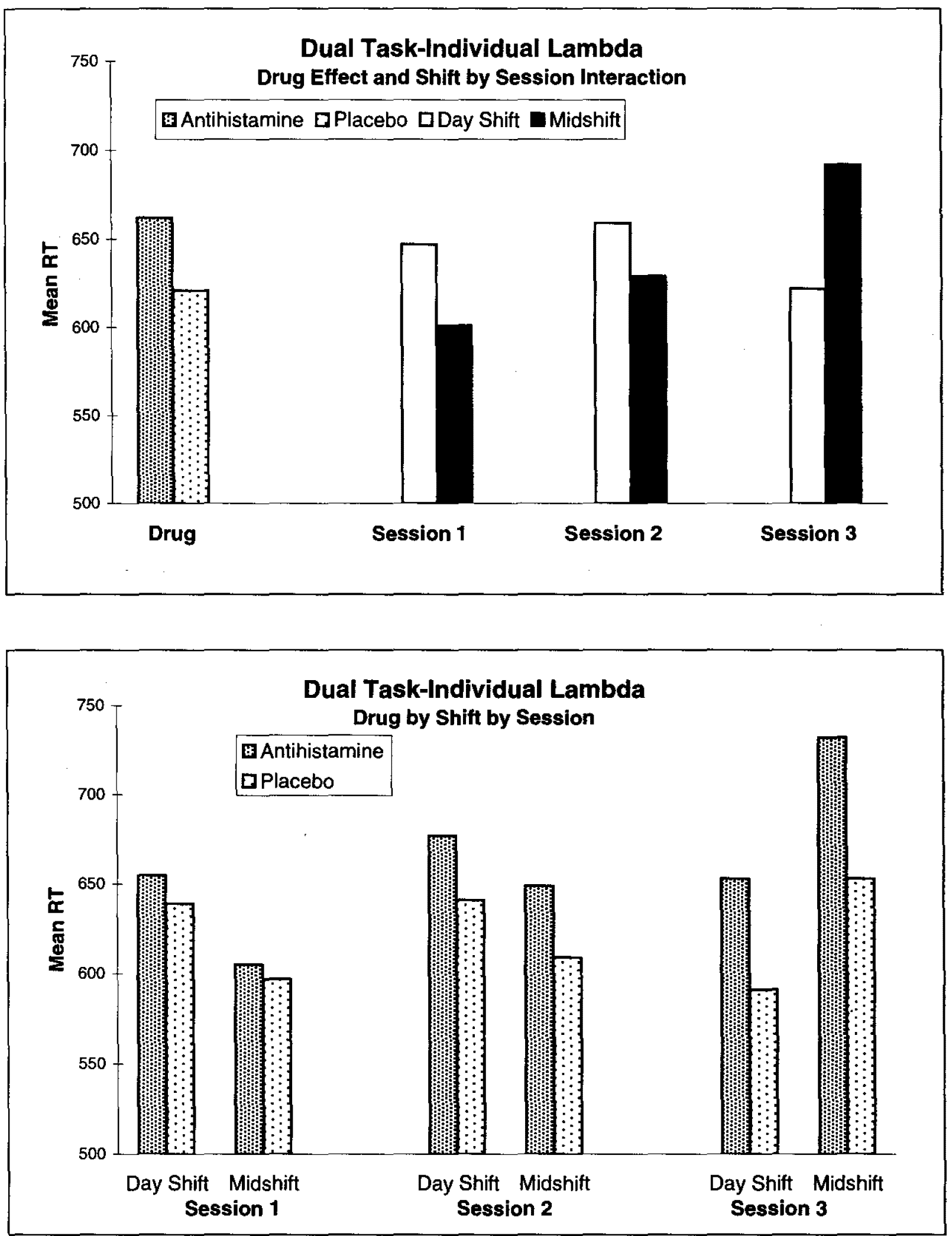

Figure 13. Dual Memory Search-Individual Lambda Mean RT. 

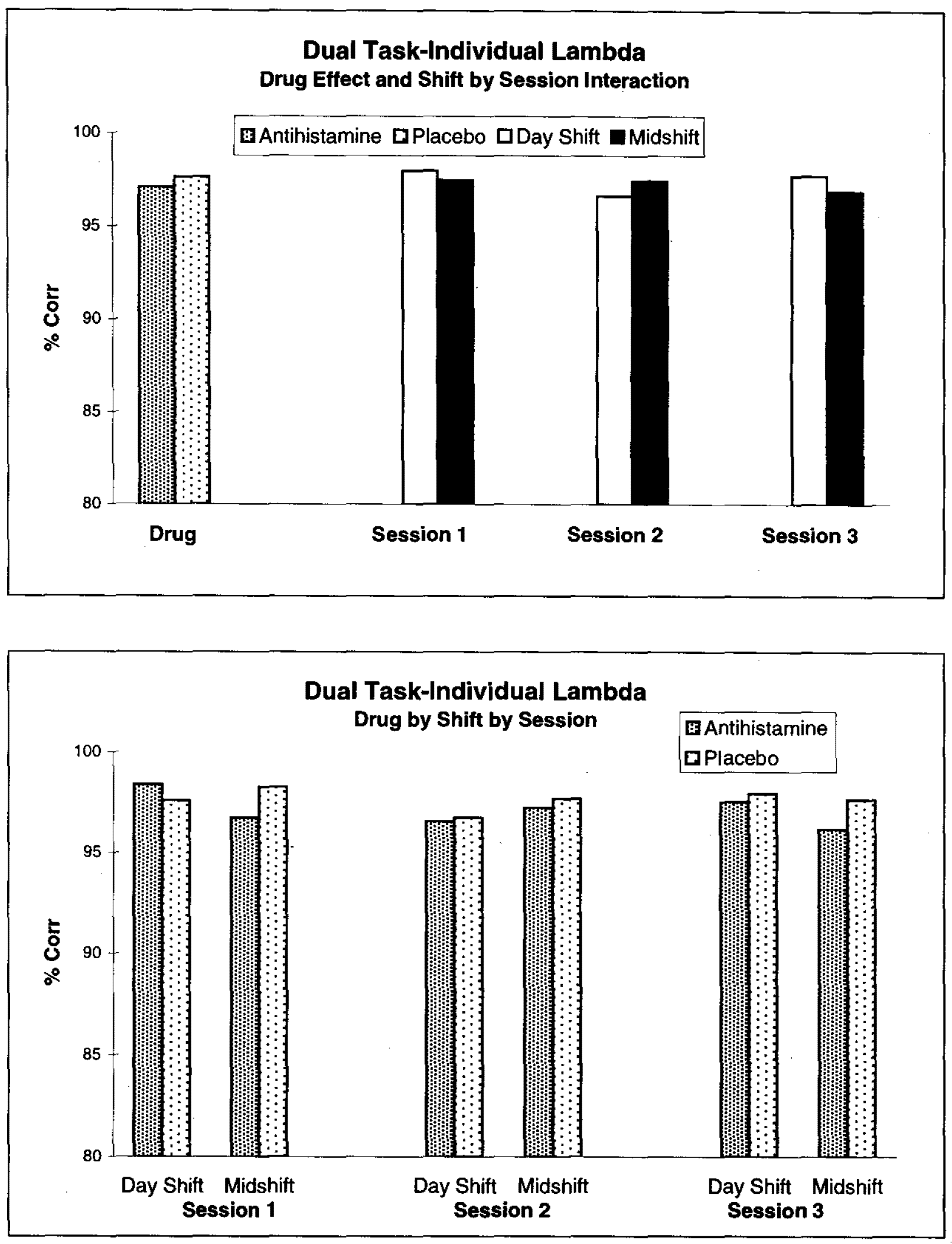

Figure 14. Dual Memory Search-Individual Lambda Percent Correct. 

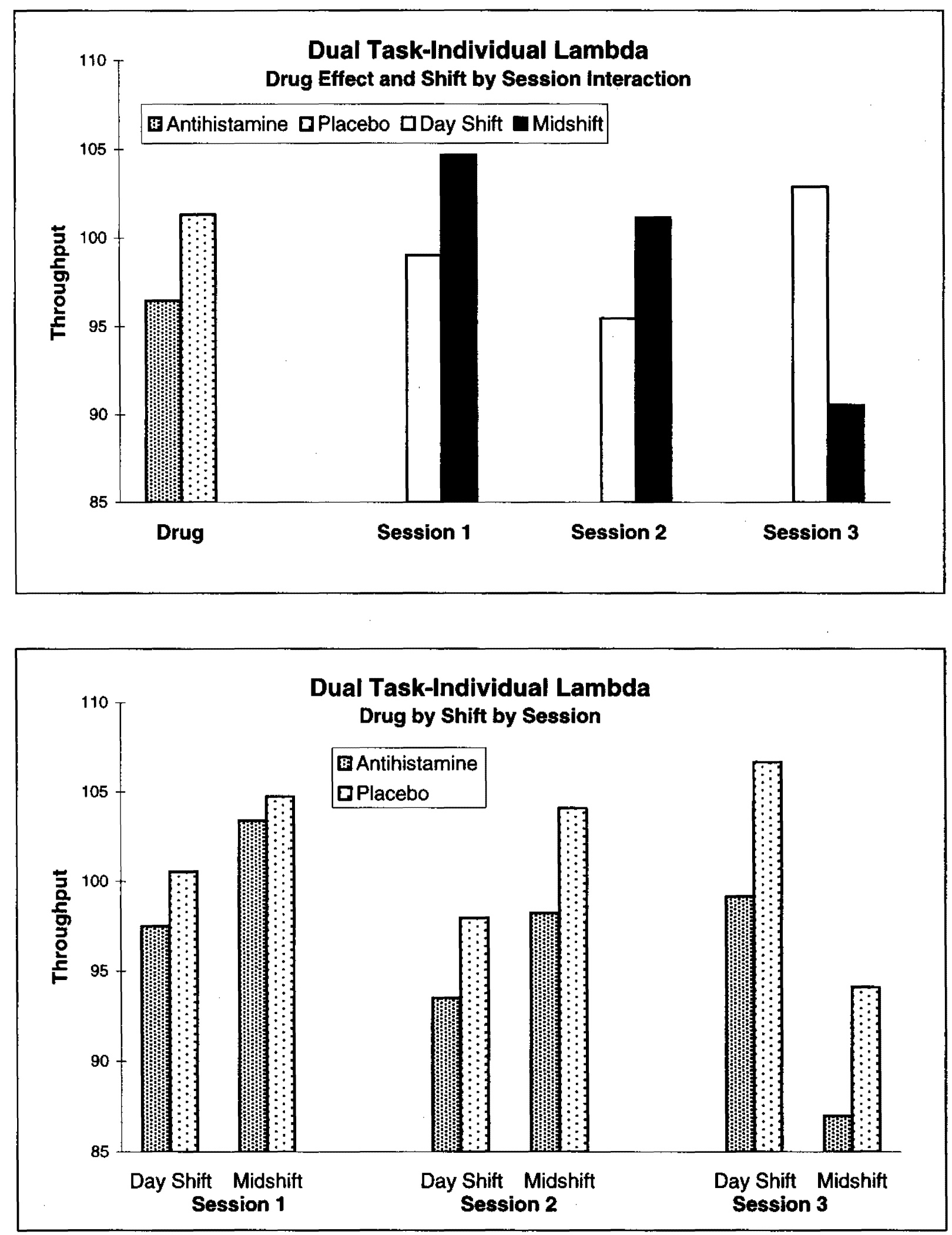

Figure 15. Dual Memory Search-Individual Lambda Throughput. 

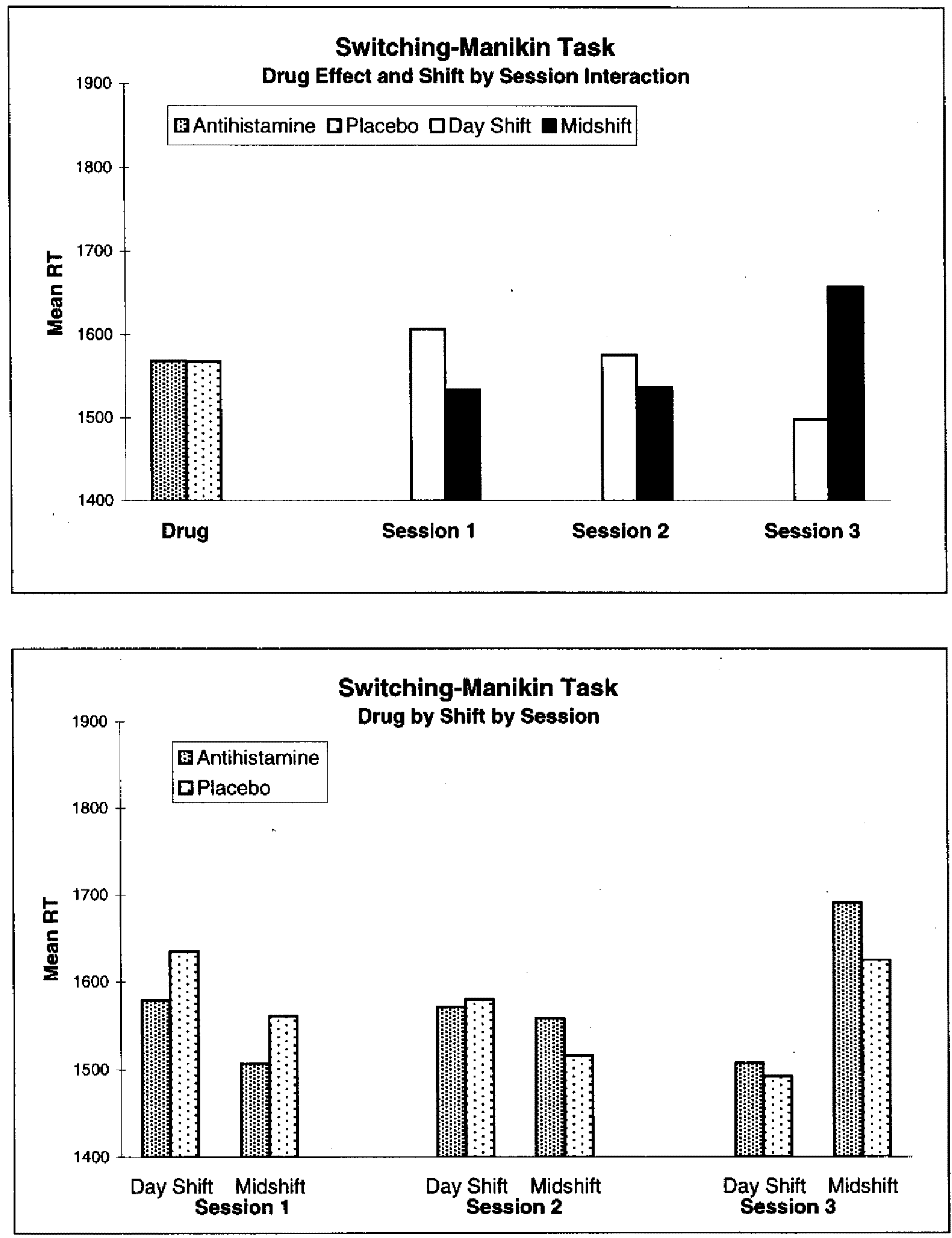

Figure 16. Switching-Manikin Task Mean RT. 

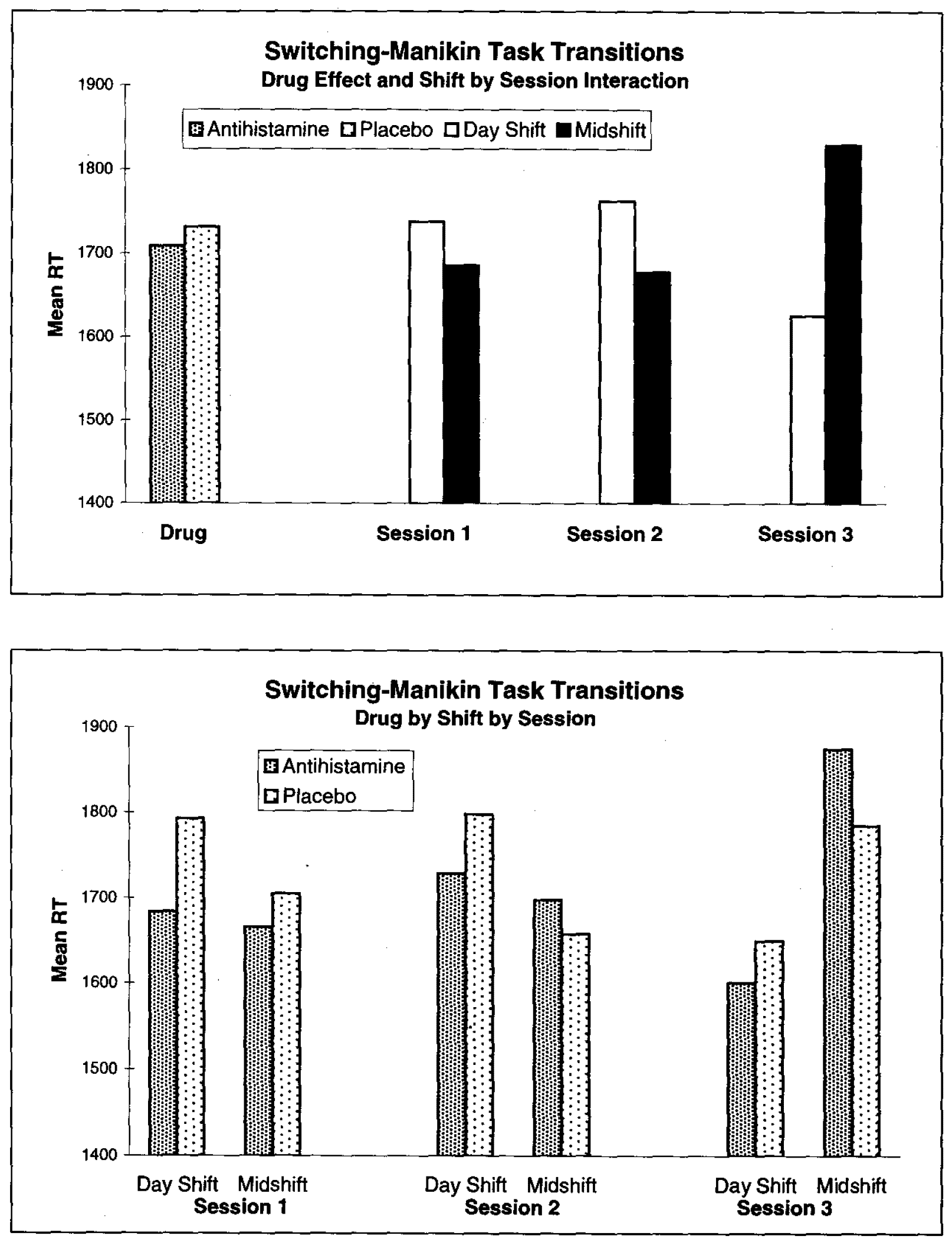

Figure 17. Switching-Manikin Task Mean RT for Transitions. 

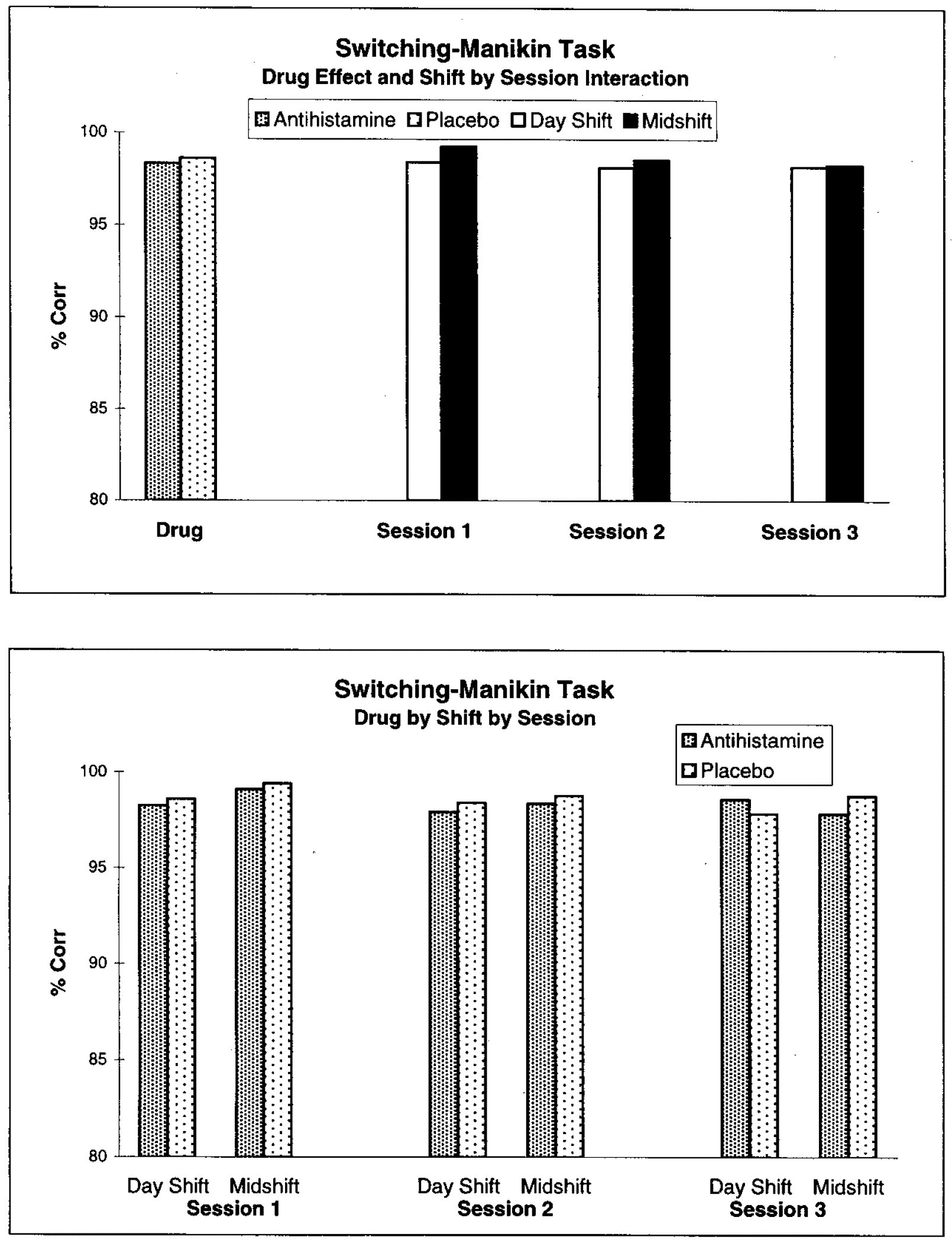

Figure 18. Switching-Manikin Task Percent Correct. 

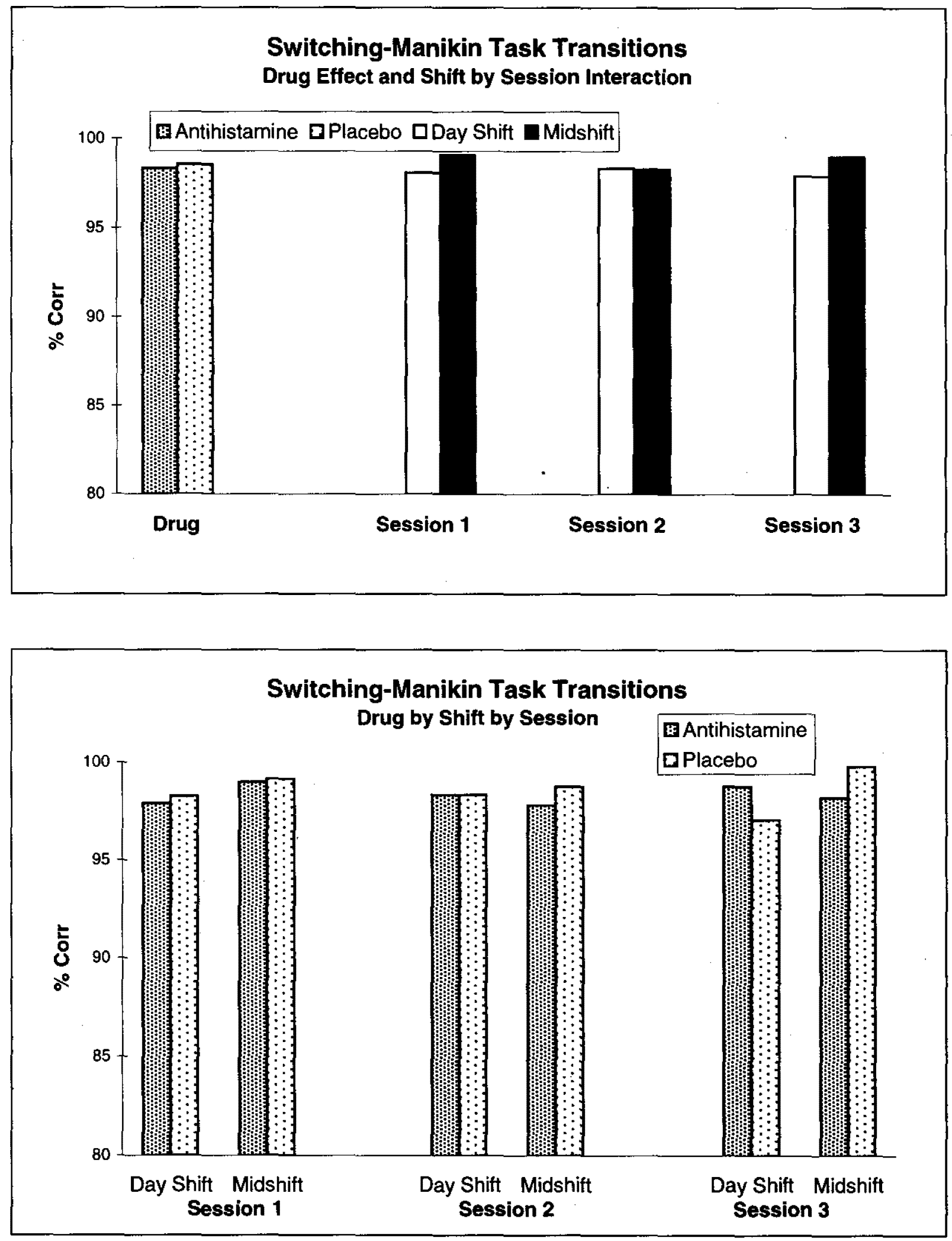

Figure 19. Switching-Manikin Task Percent Correct for Transitions. 

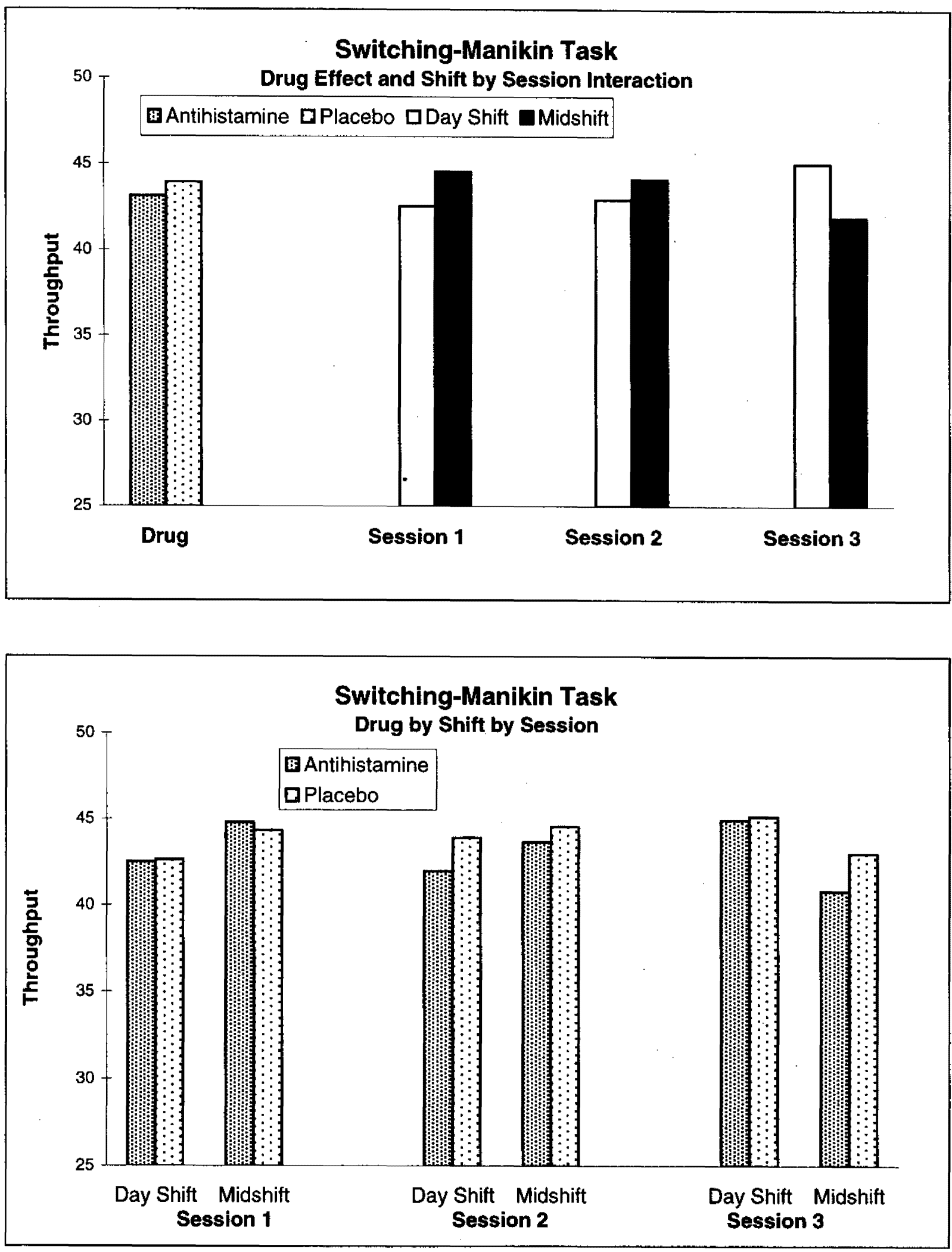

Figure 20. Switching-Manikin Task Throughput. 

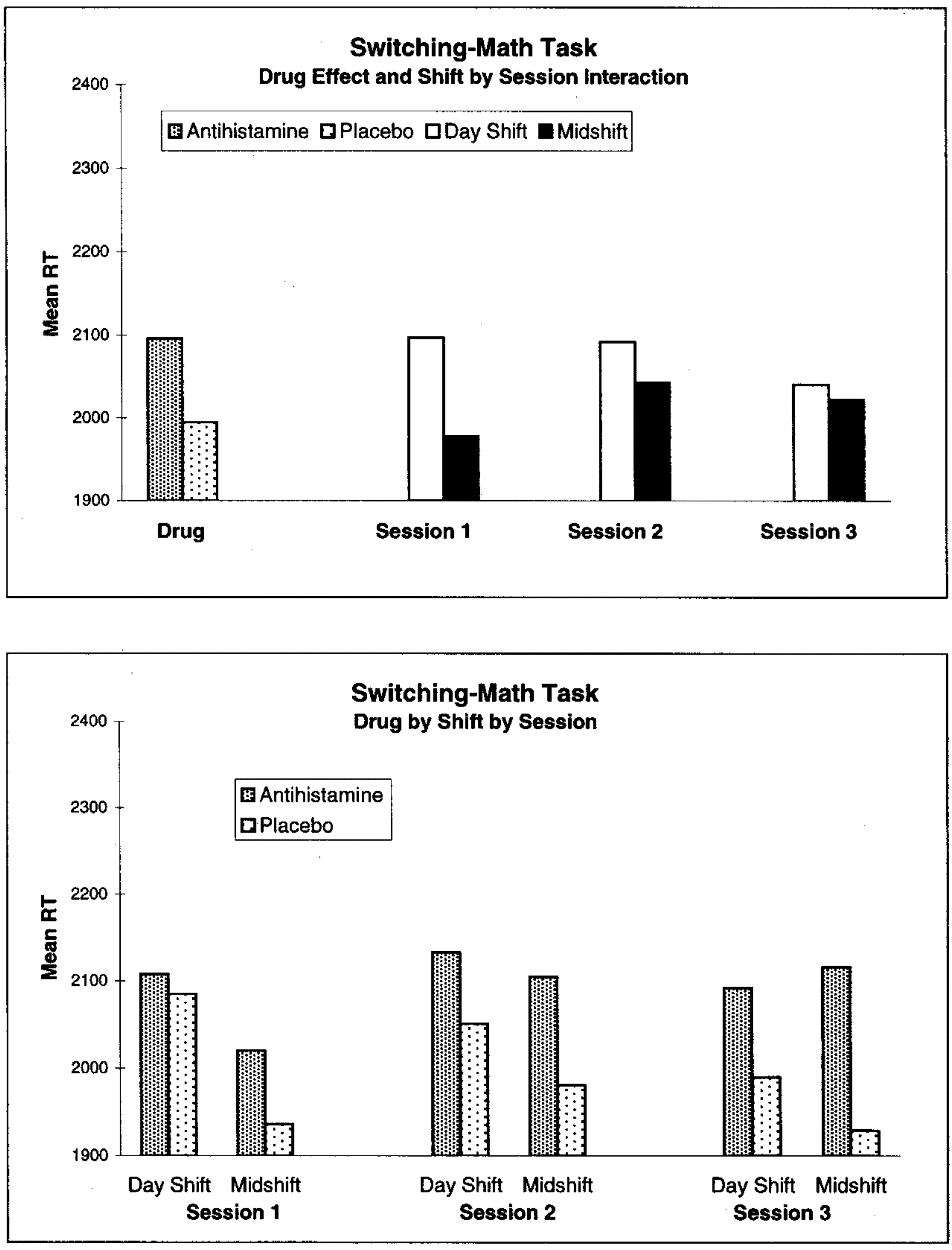

Figure 21. Switching-Mathematical Processing Mean RT. 

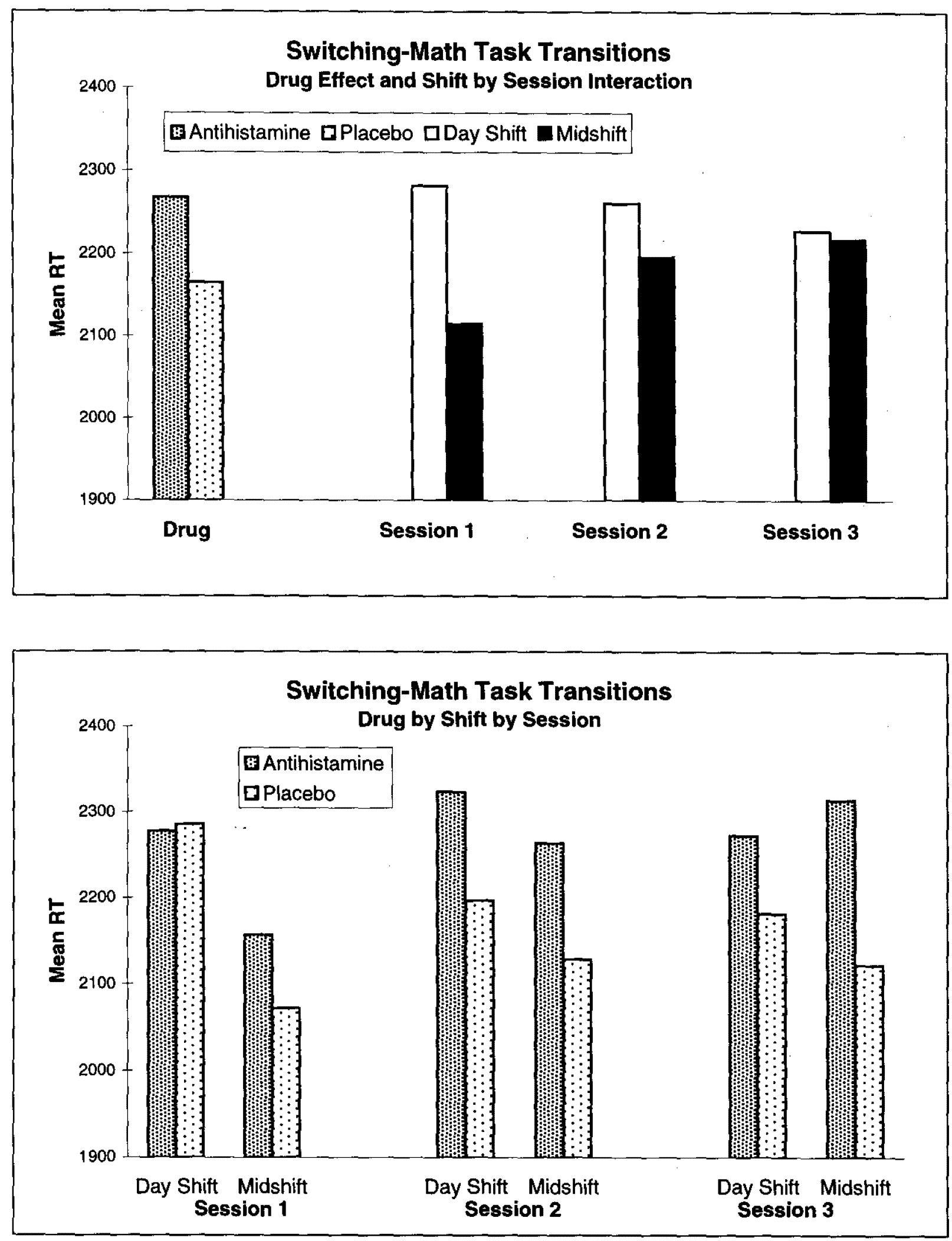

Figure 22. Switching-Mathematical Processing Mean RT for Transitions. 

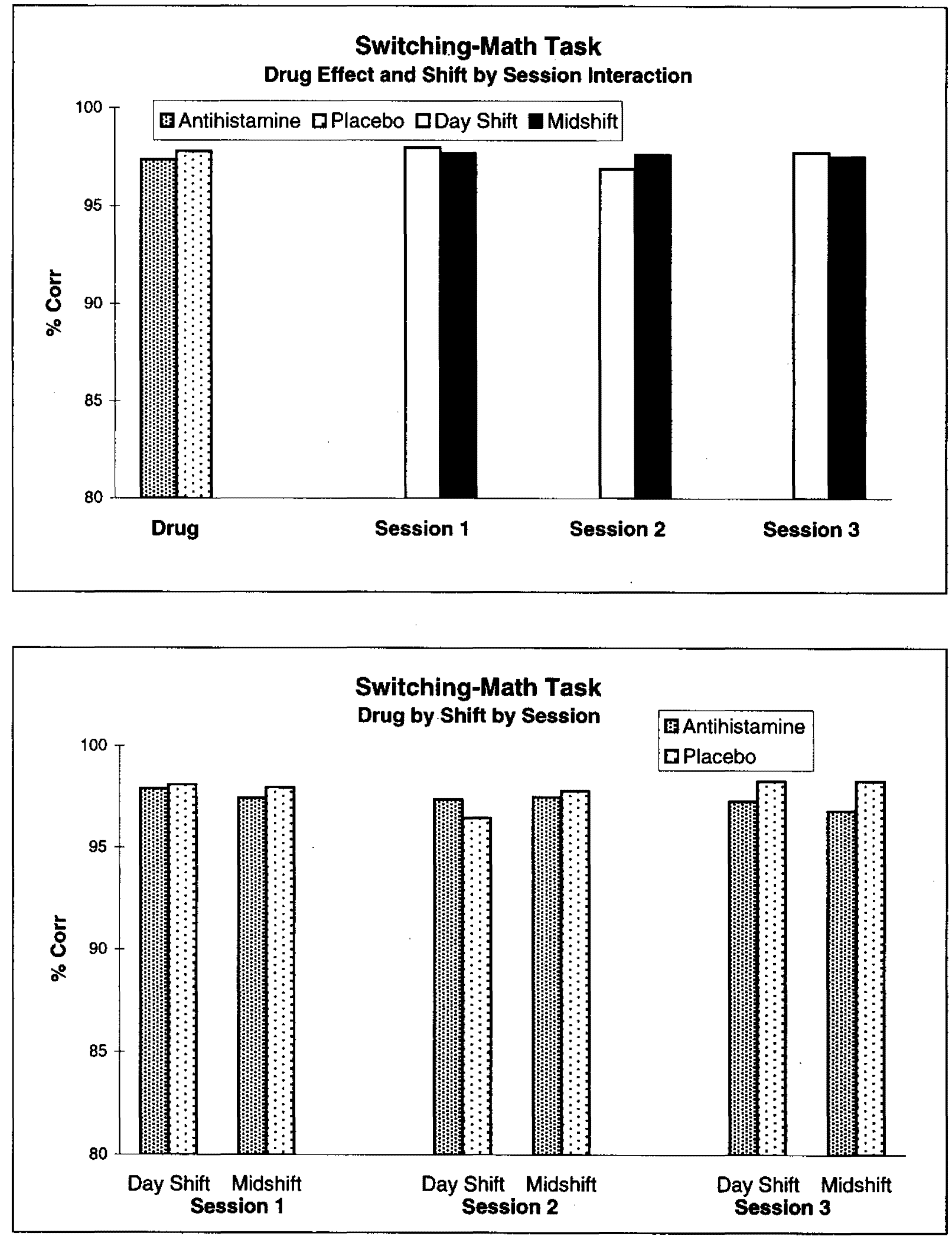

Figure 23. Switching-Mathematical Processing Percent Correct. 

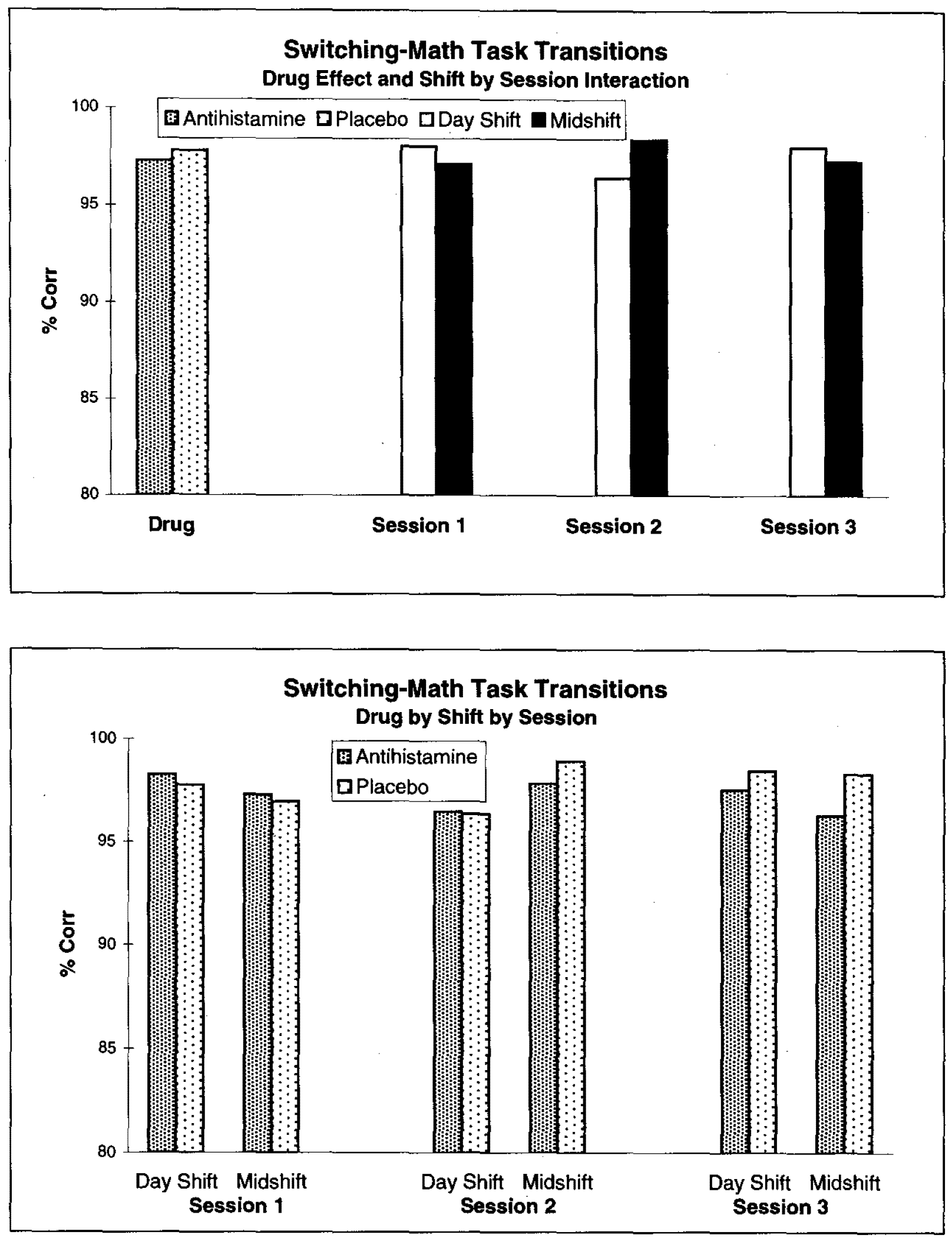

Figure 24. Switching-Mathematical Processing Percent Correct for Transitions. 

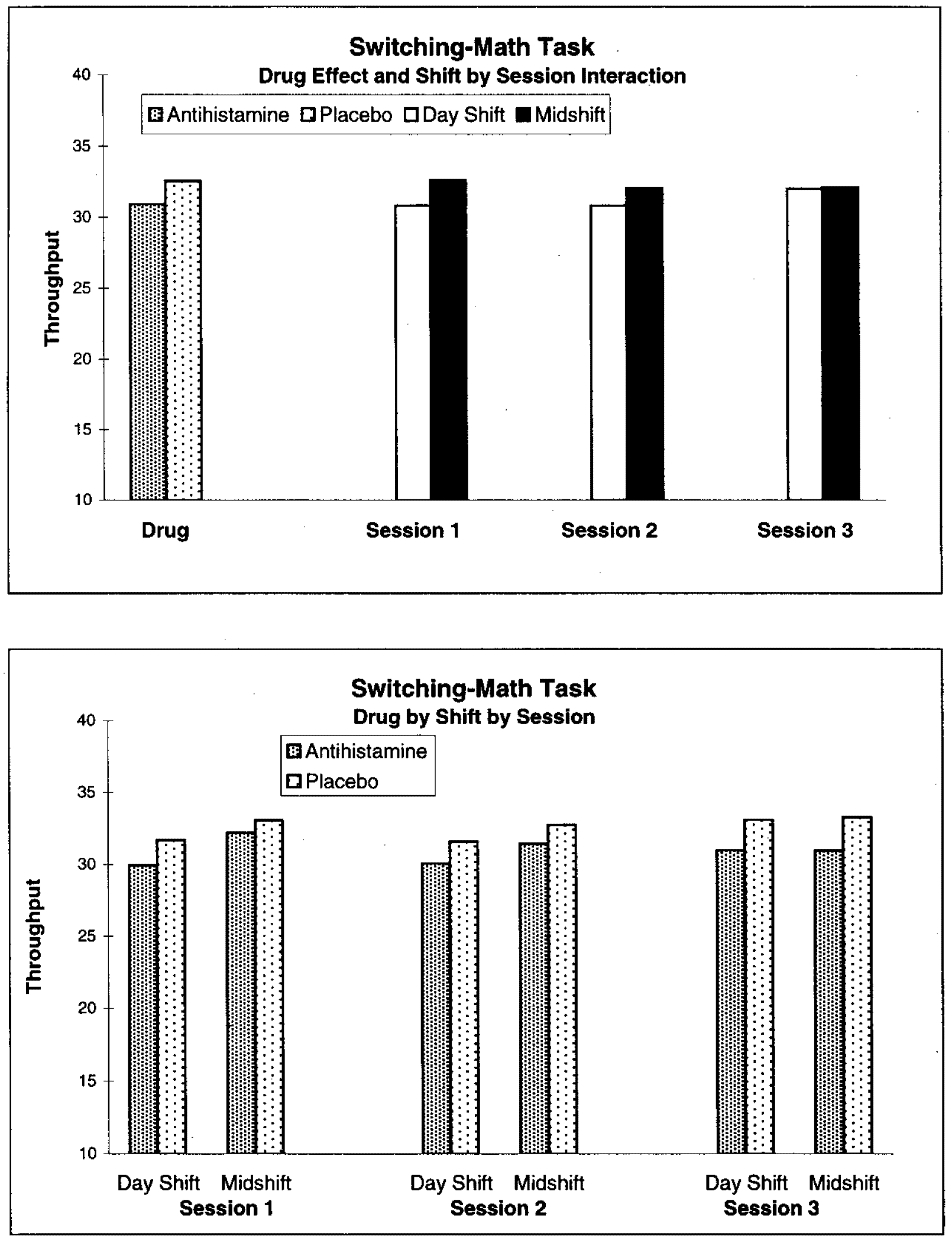

Figure 25. Switching-Mathematical Processing Throughput. 

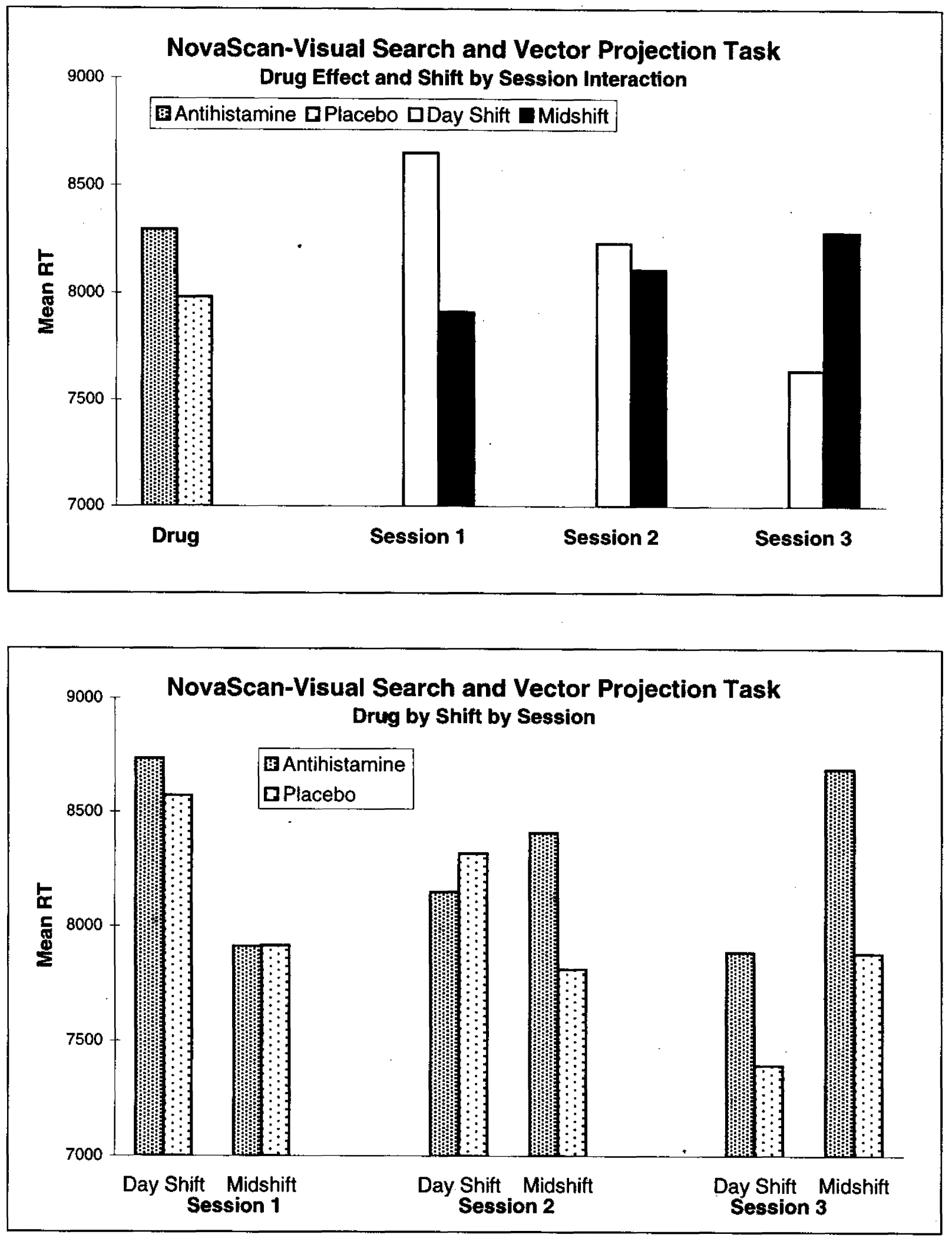

Figure 26. NovaScan-Visual Search and Vector Projection Task Mean RT. 

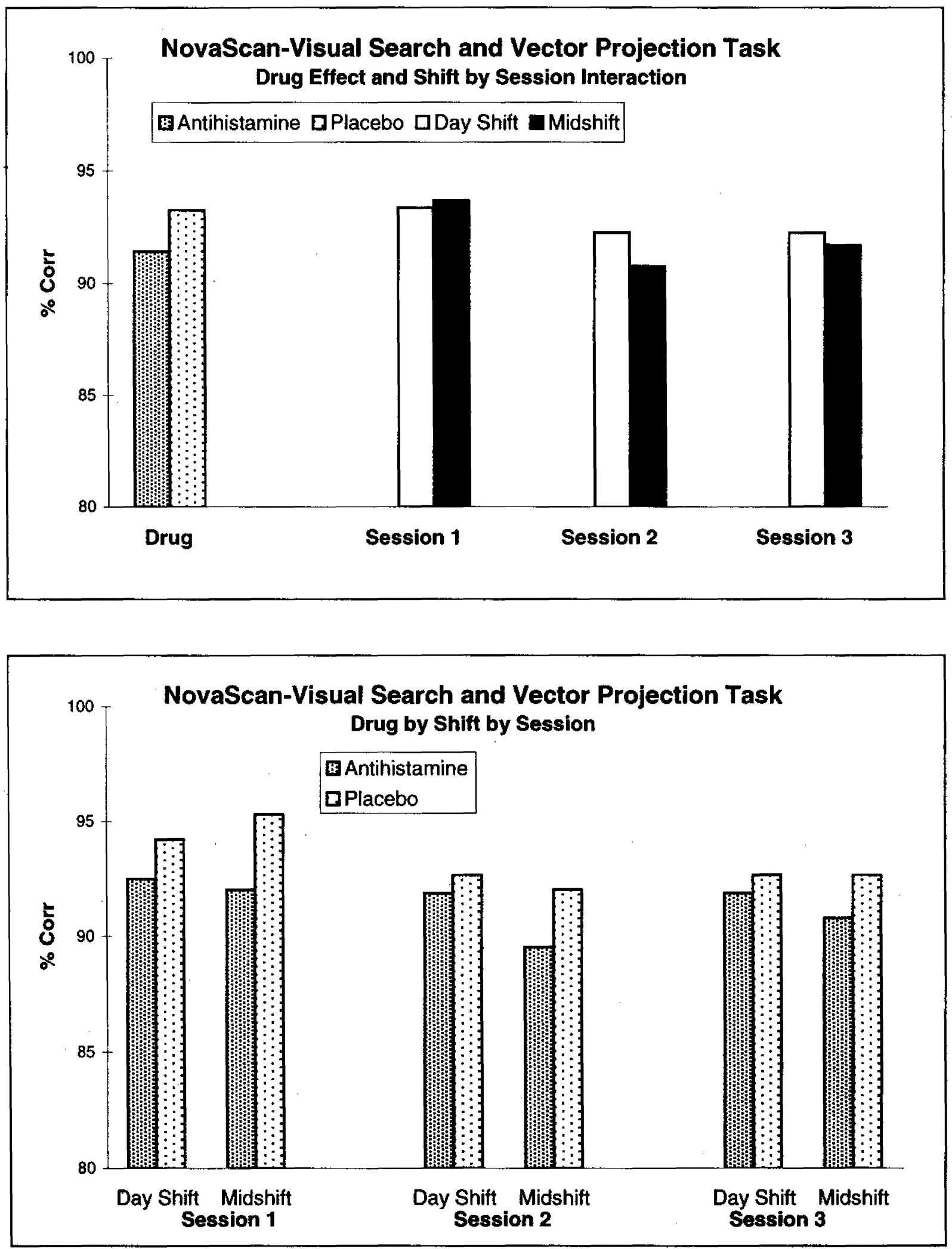

Figure 27. NovaScan-Visual Search and Vector Projection Percent Correct. 

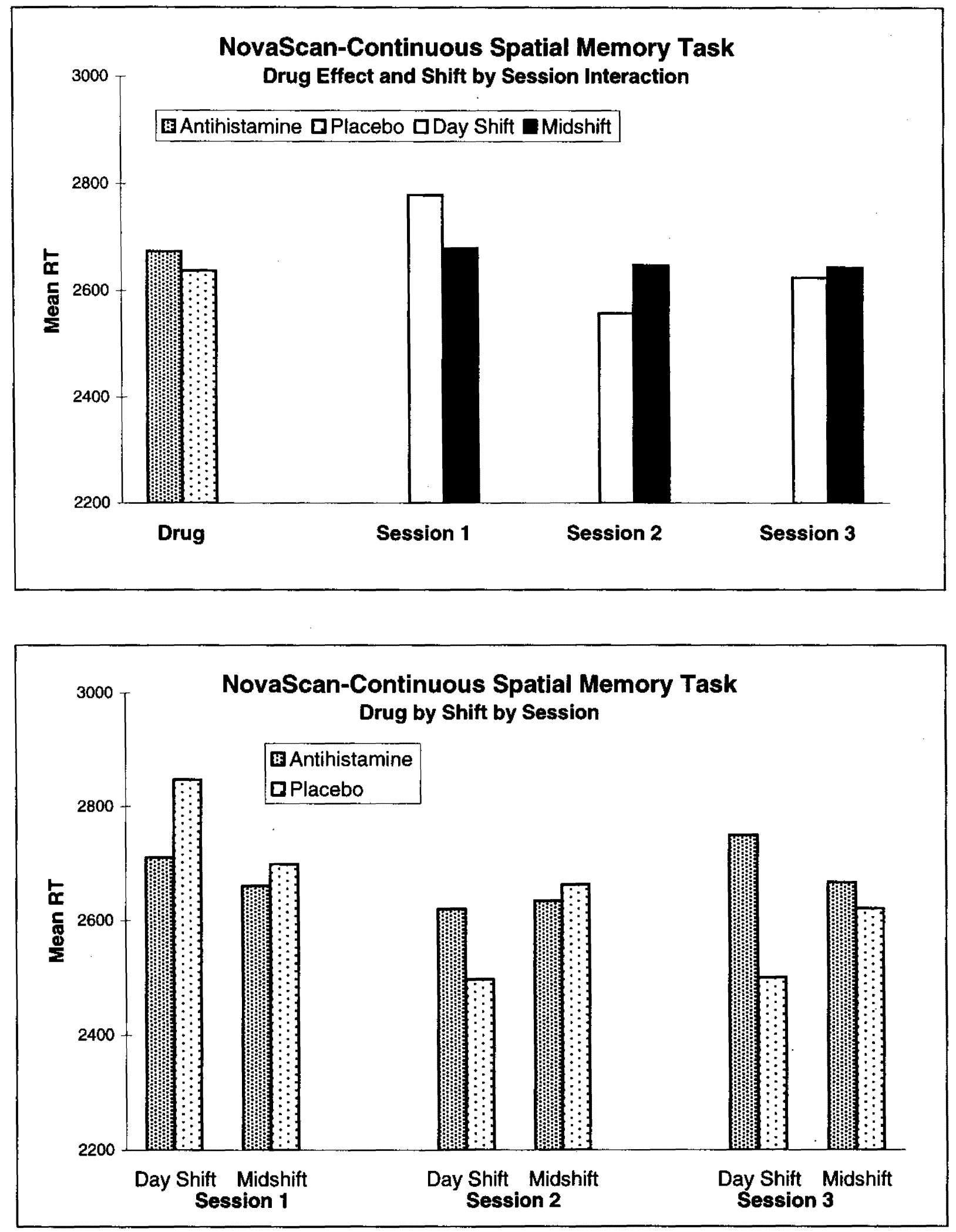

Figure 28. NovaScan-Continuous Spatial Memory Task Mean RT. 

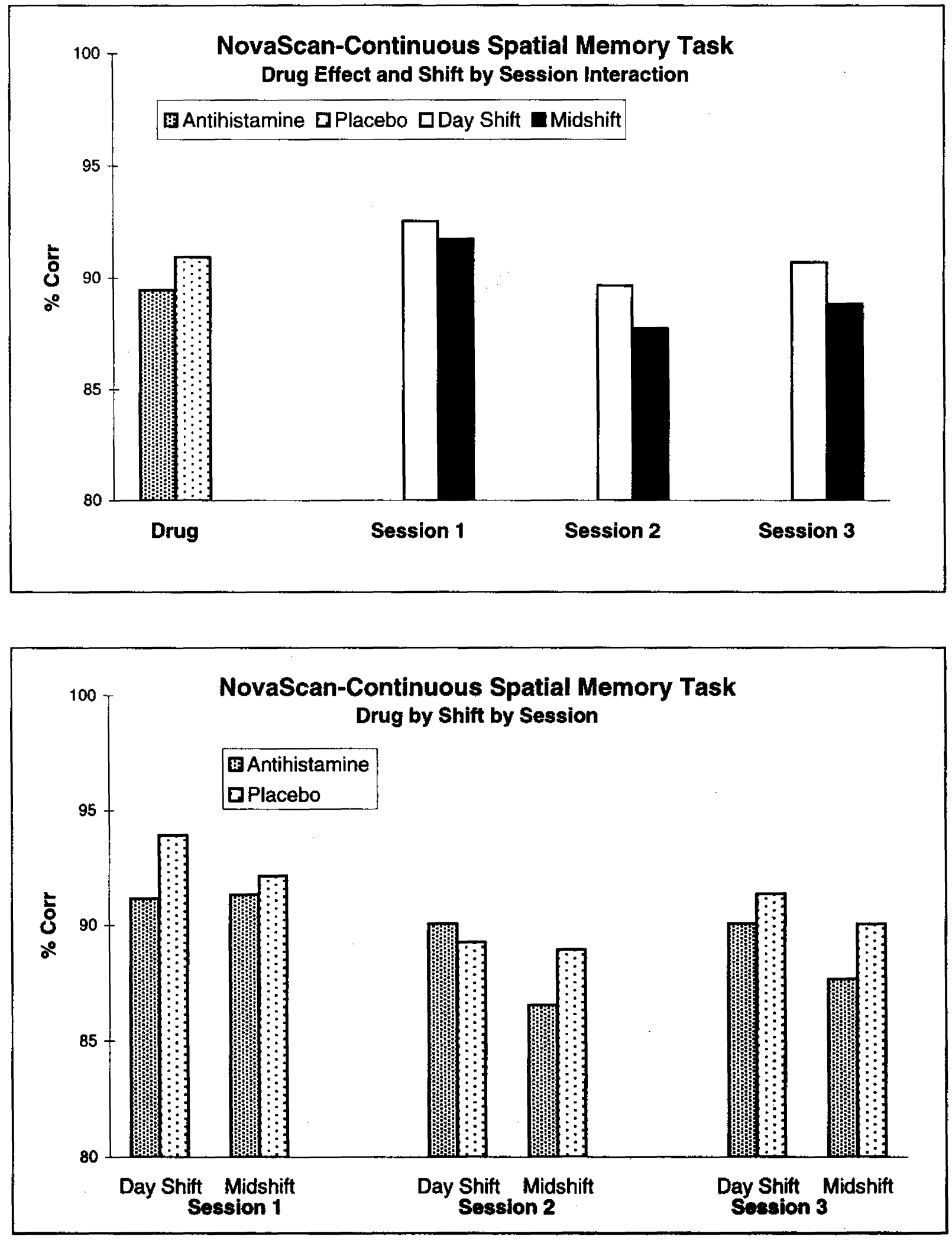

Figure 29. NovaScan-Continuous Spatial Memory Task Percent Correct. 

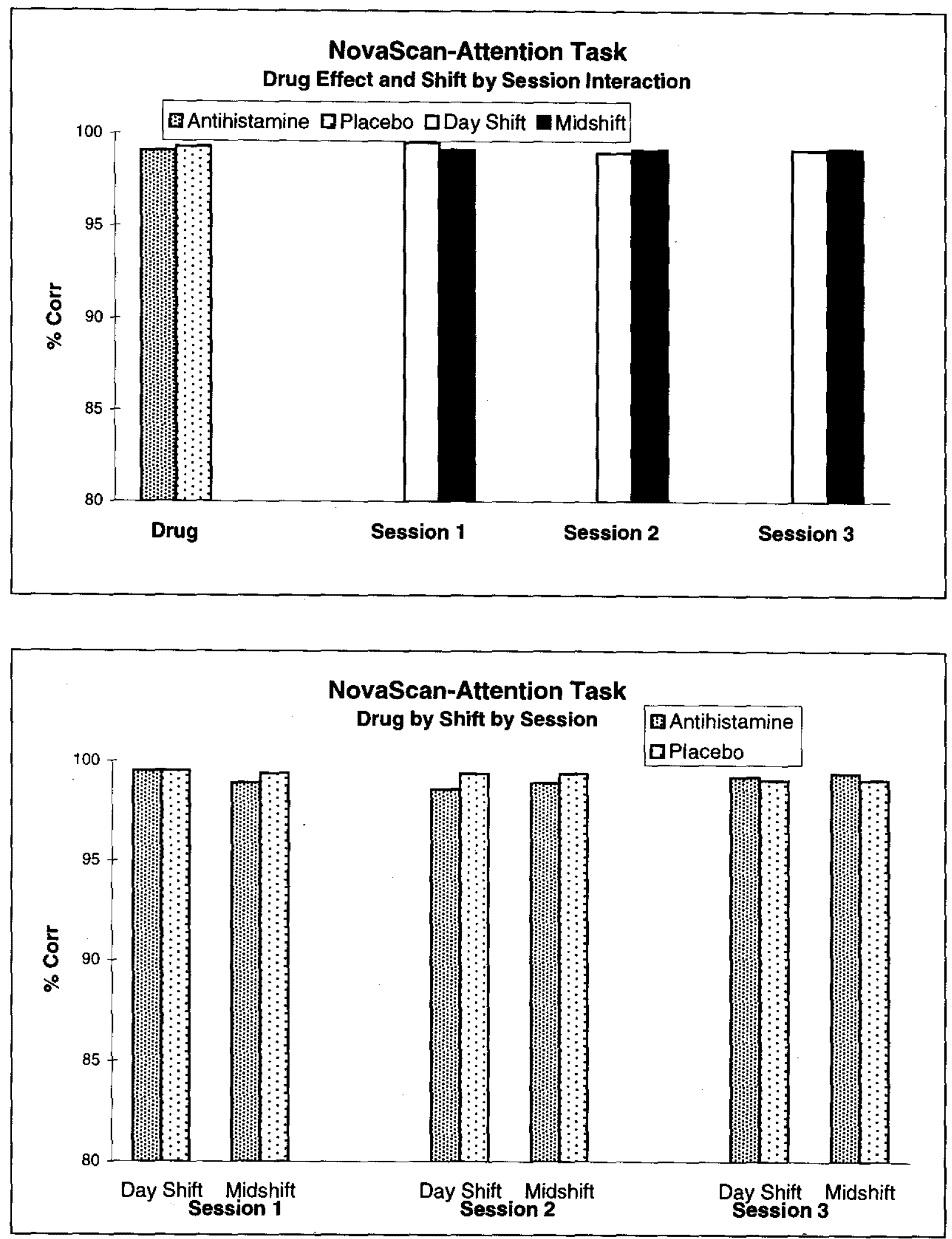

Figure 30. NovaScan-Attention Task Percent Correct. 

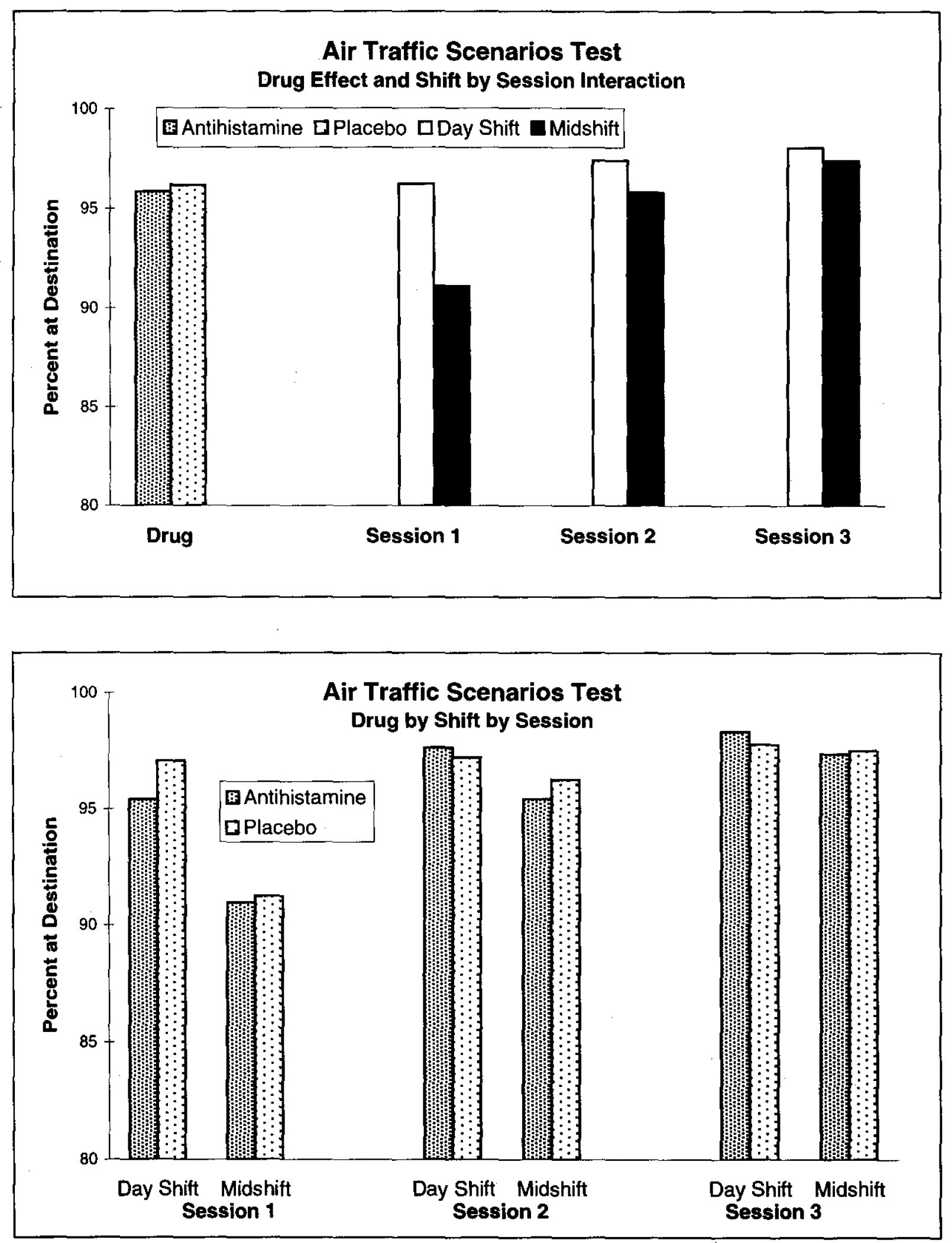

Figure 31. Air Traffic Scenarios Test Percent at Destination. 

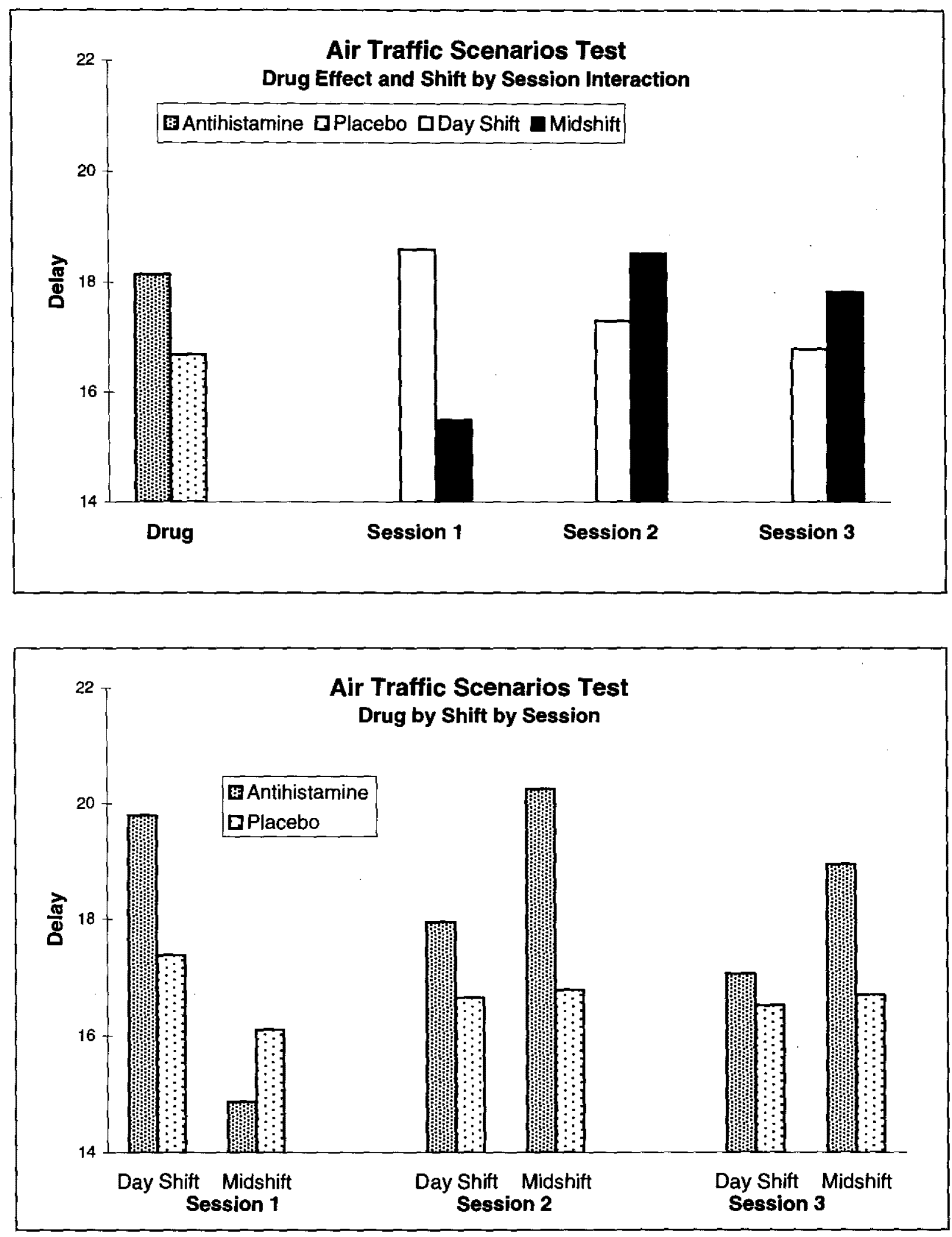

Figure 32. Air Traffic Scenarios Test Delay. 

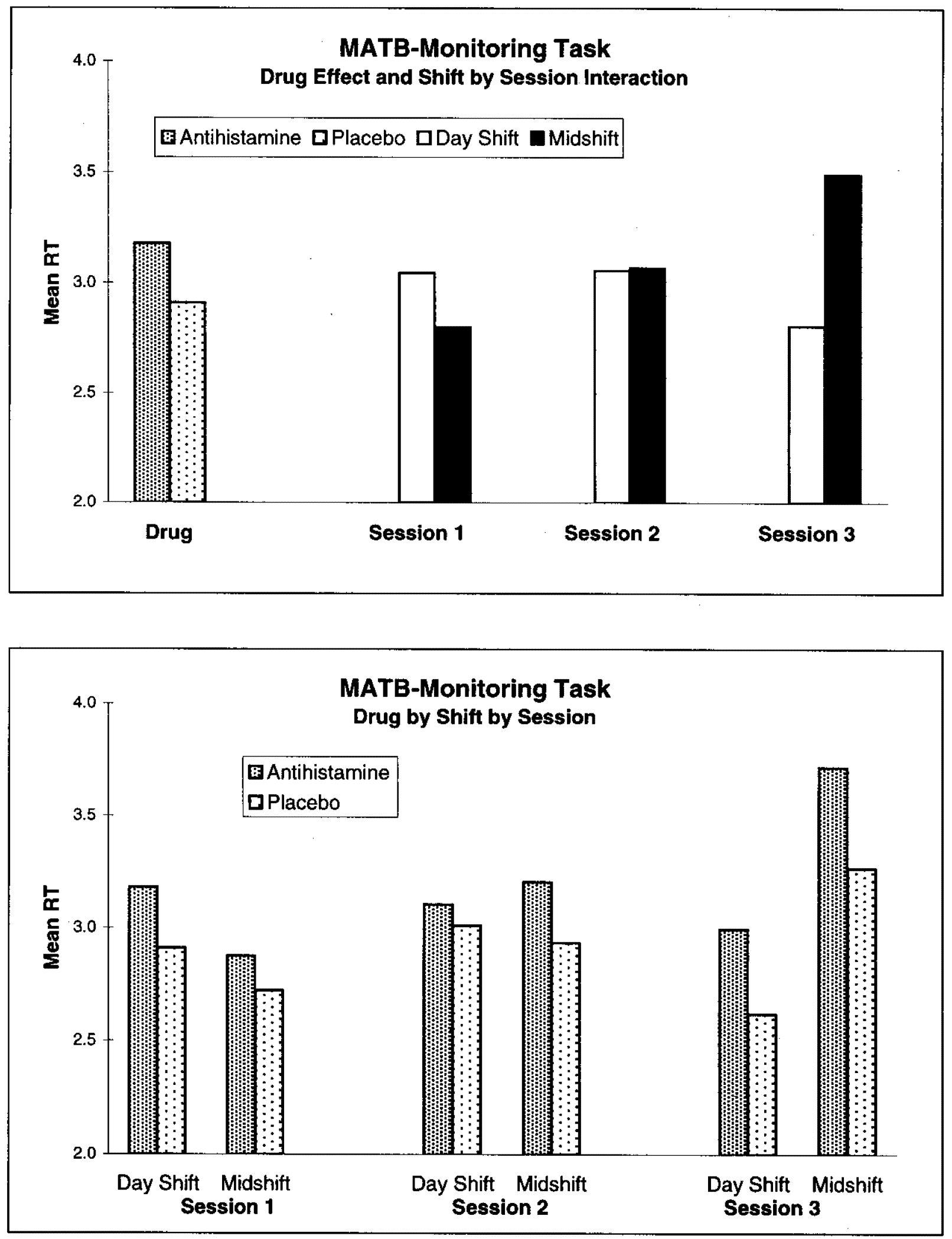

Figure 33. MATB-Monitoring Task Mean RT. 

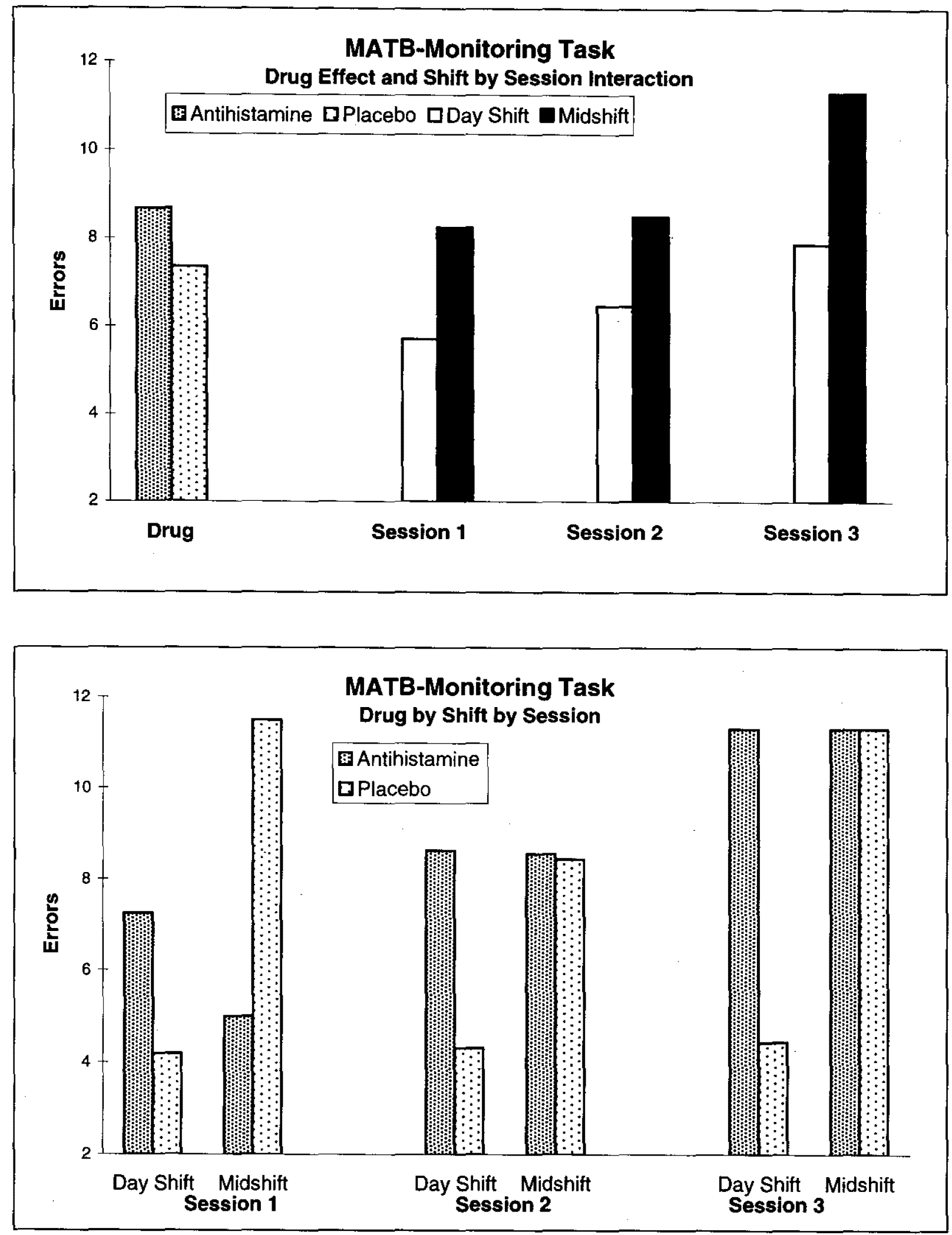

Figure 34. MATB-Monitoring Task Errors. 

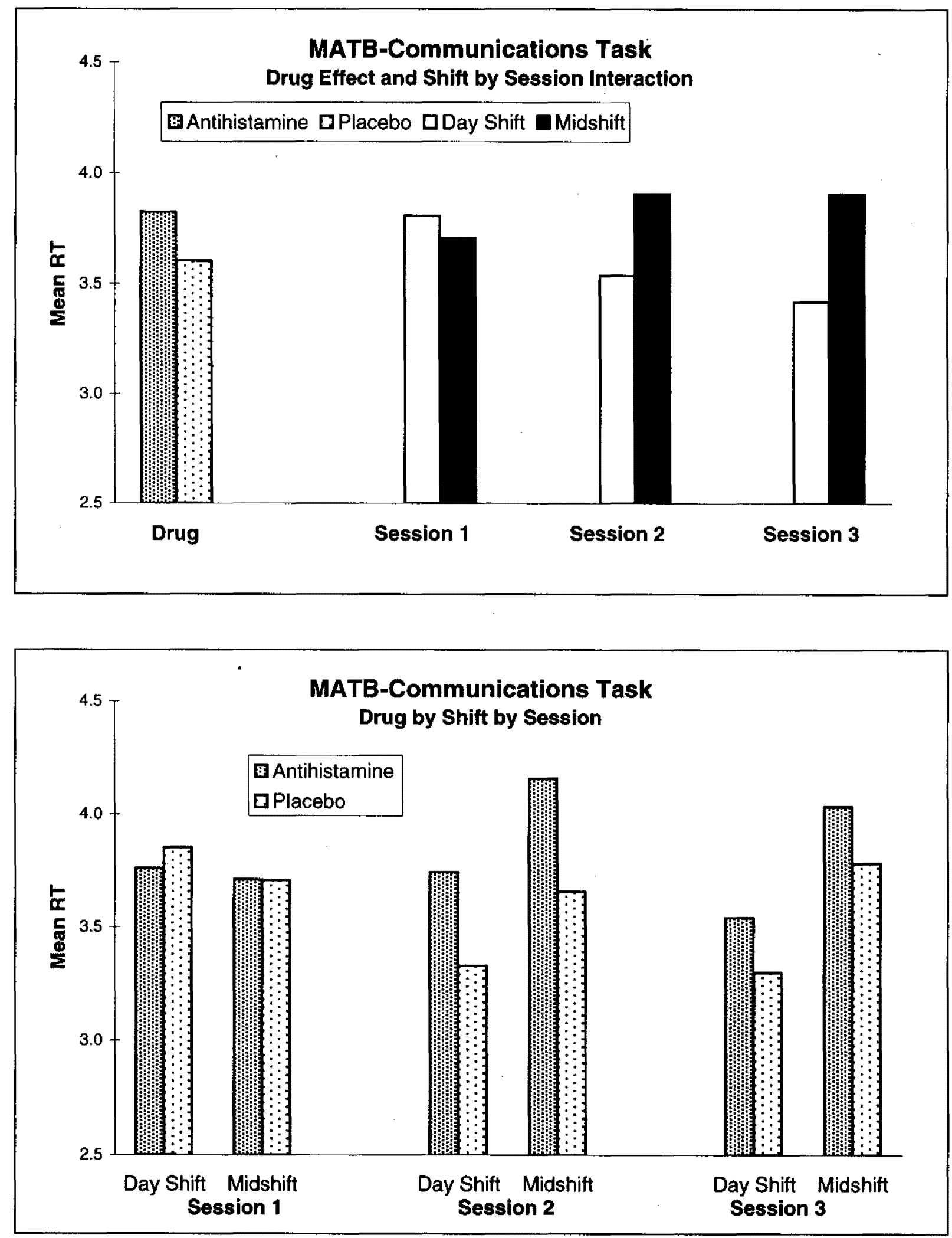

Figure 35. MATB-Communications Task Mean RT. 

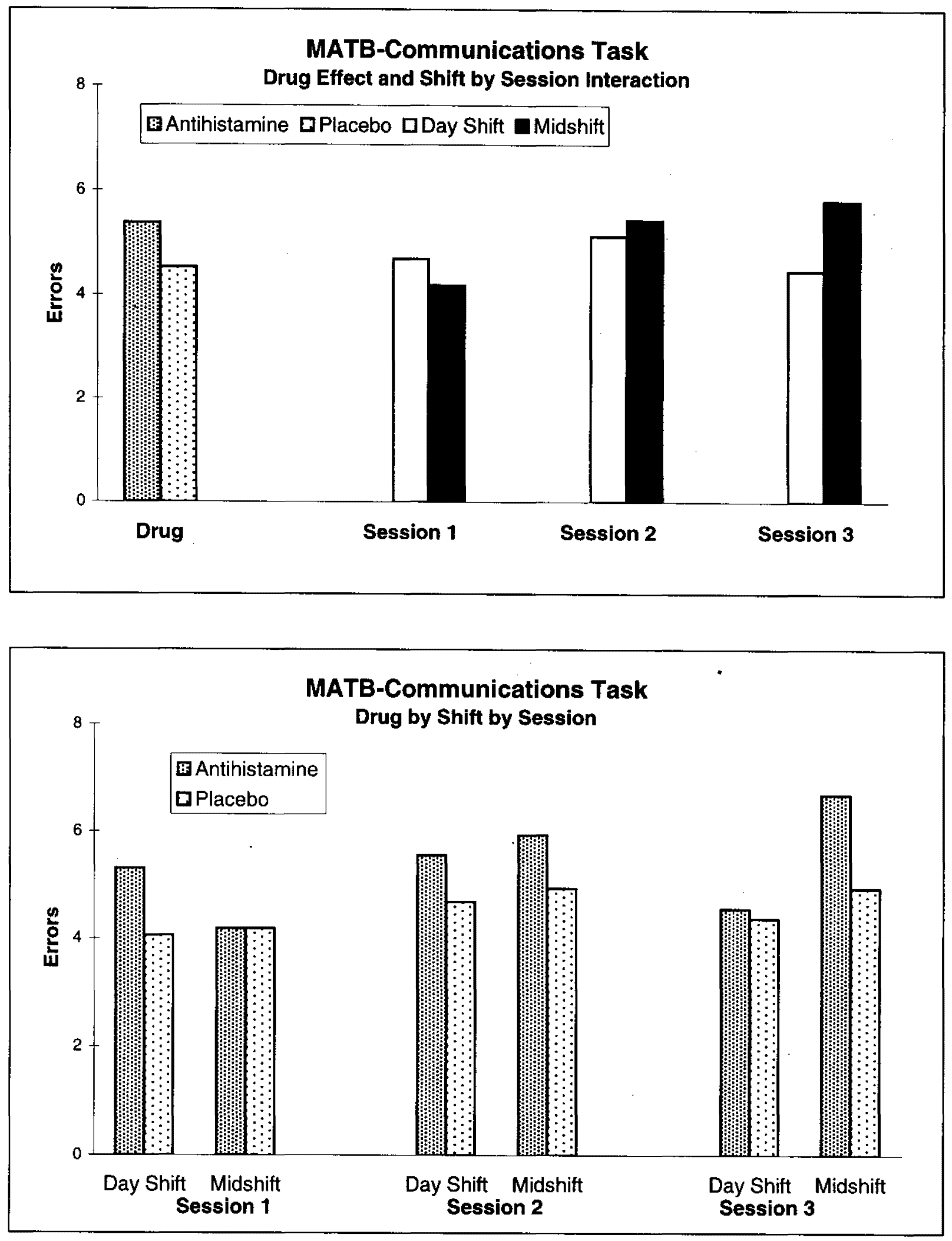

Figure 36. MATB-Communications Task Errors. 

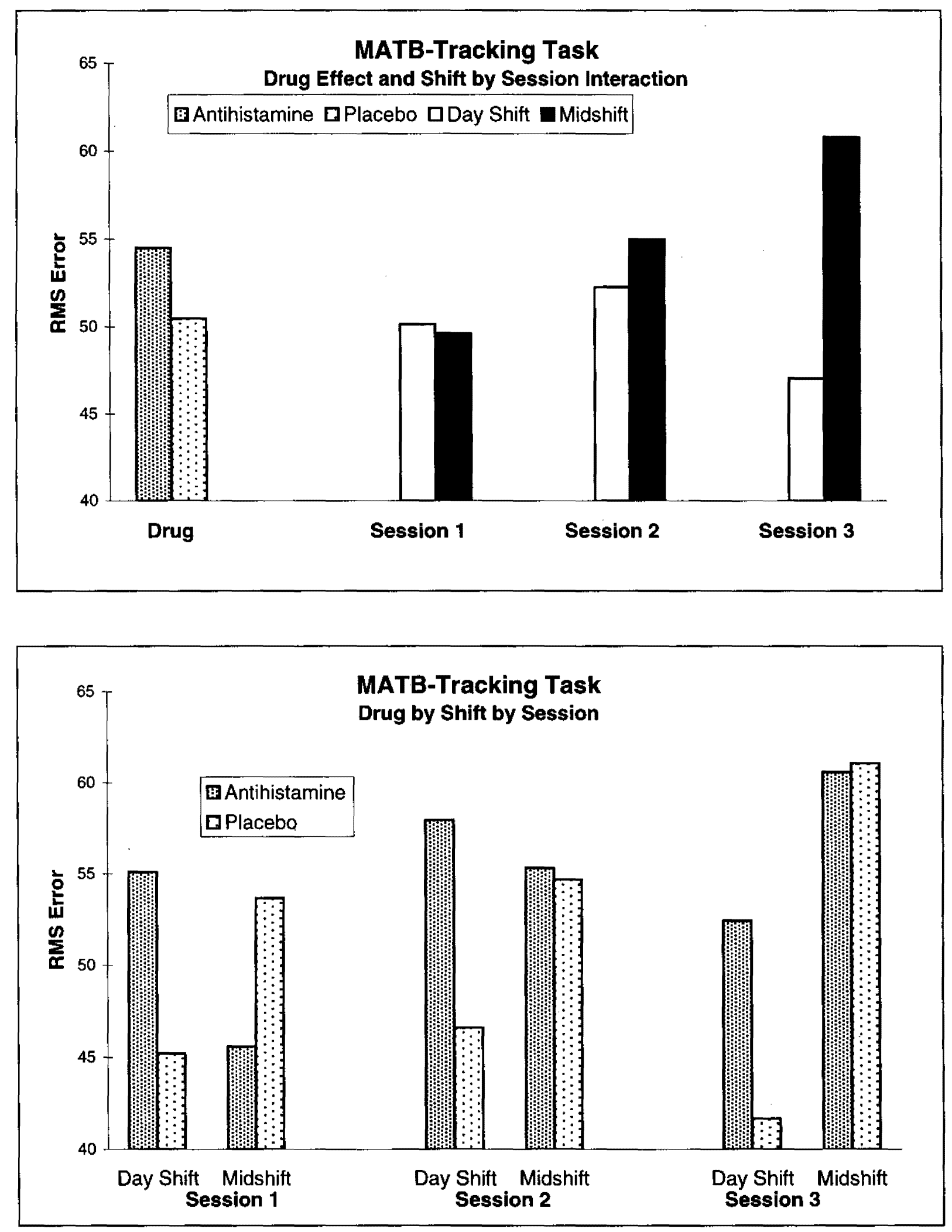

Figure 37. MATB-Tracking RMS Error. 

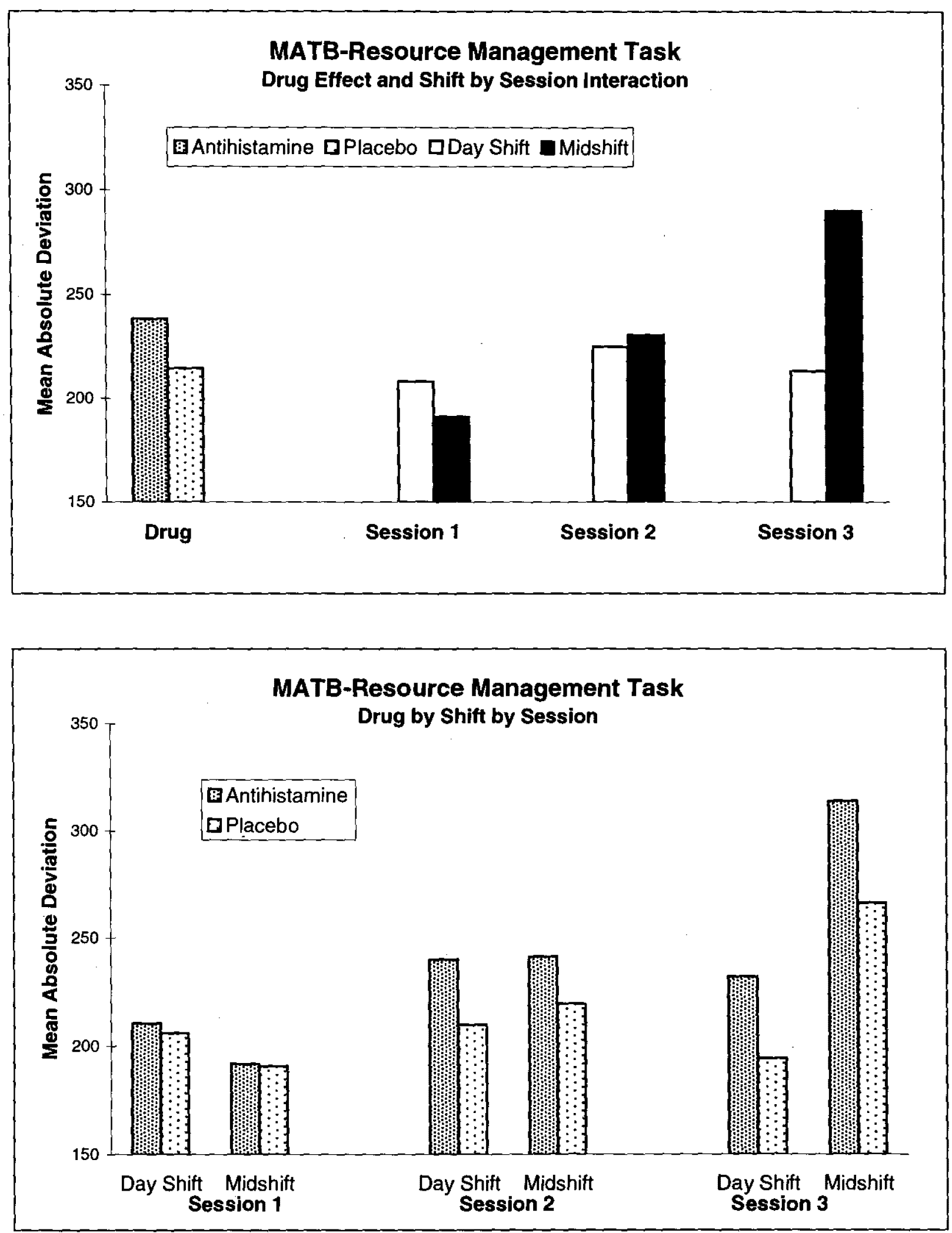

Figure 38. MATB-Resource Management Task Mean Absolute Deviation. 

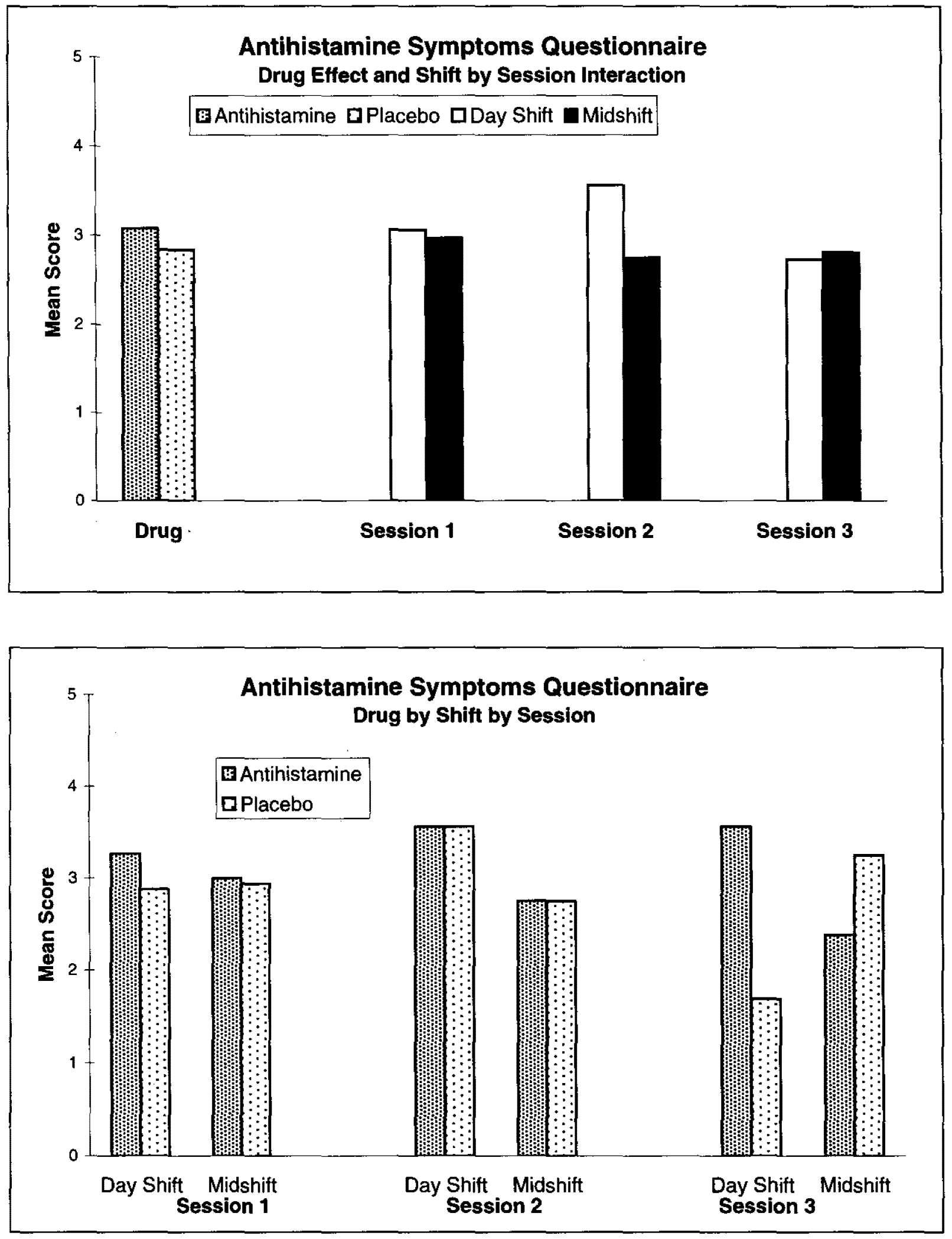

Figure 39. Antihistamine Symptoms Questionnaire Mean Score. 

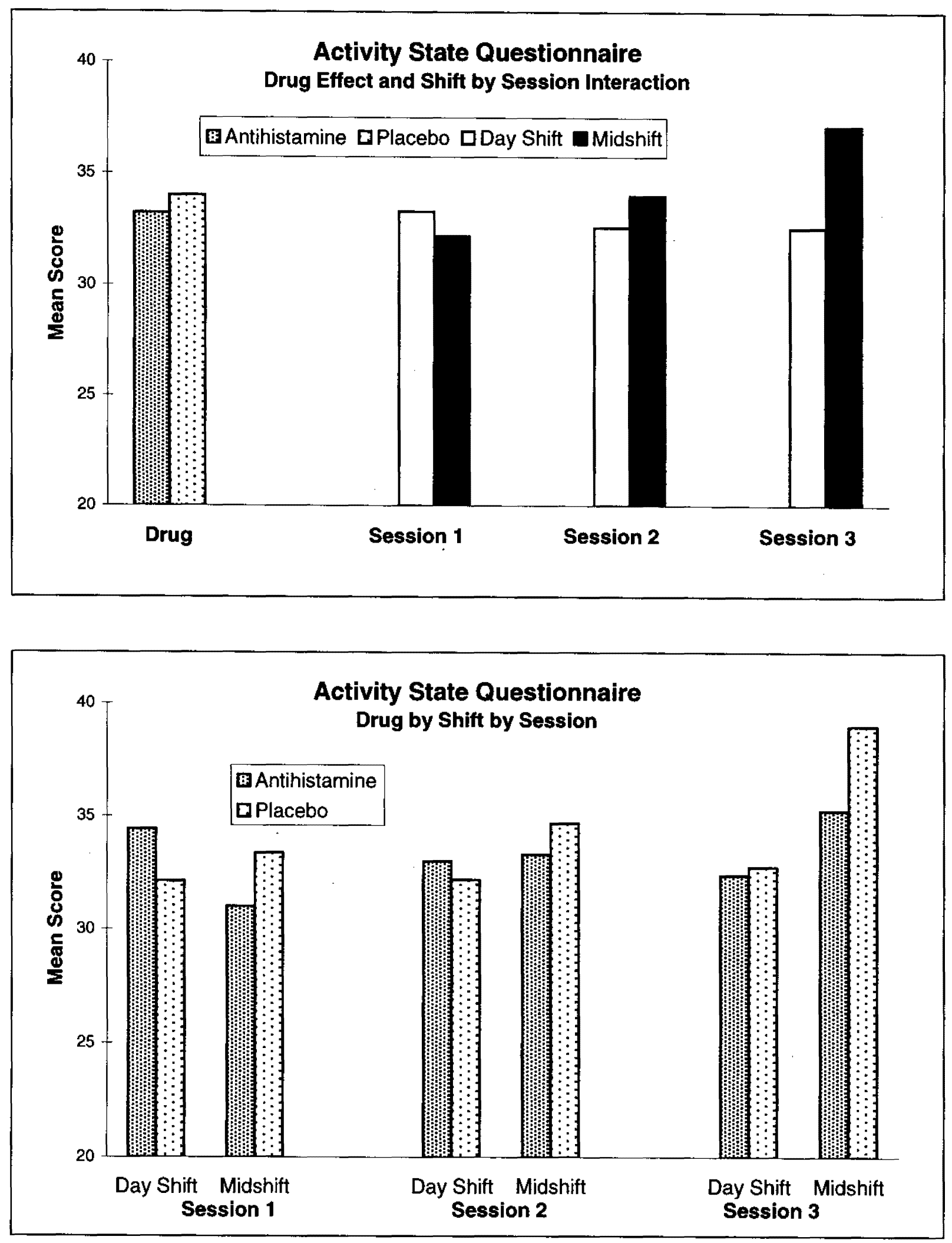

Figure 40. Activity State Questionnaire Mean PHYSICAL Score. 

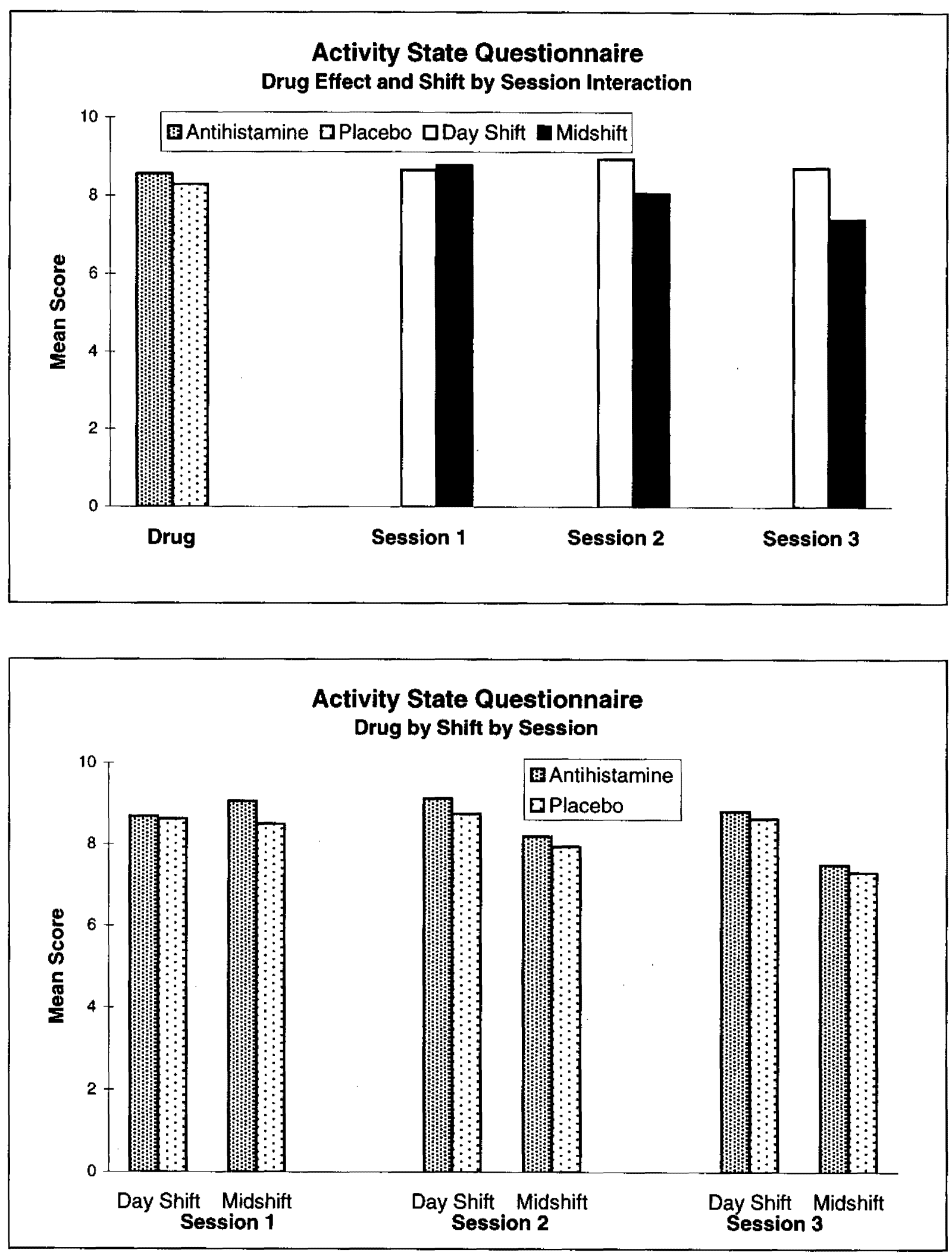

Figure 41. Activity State Questionnaire Mean PREP Score. 

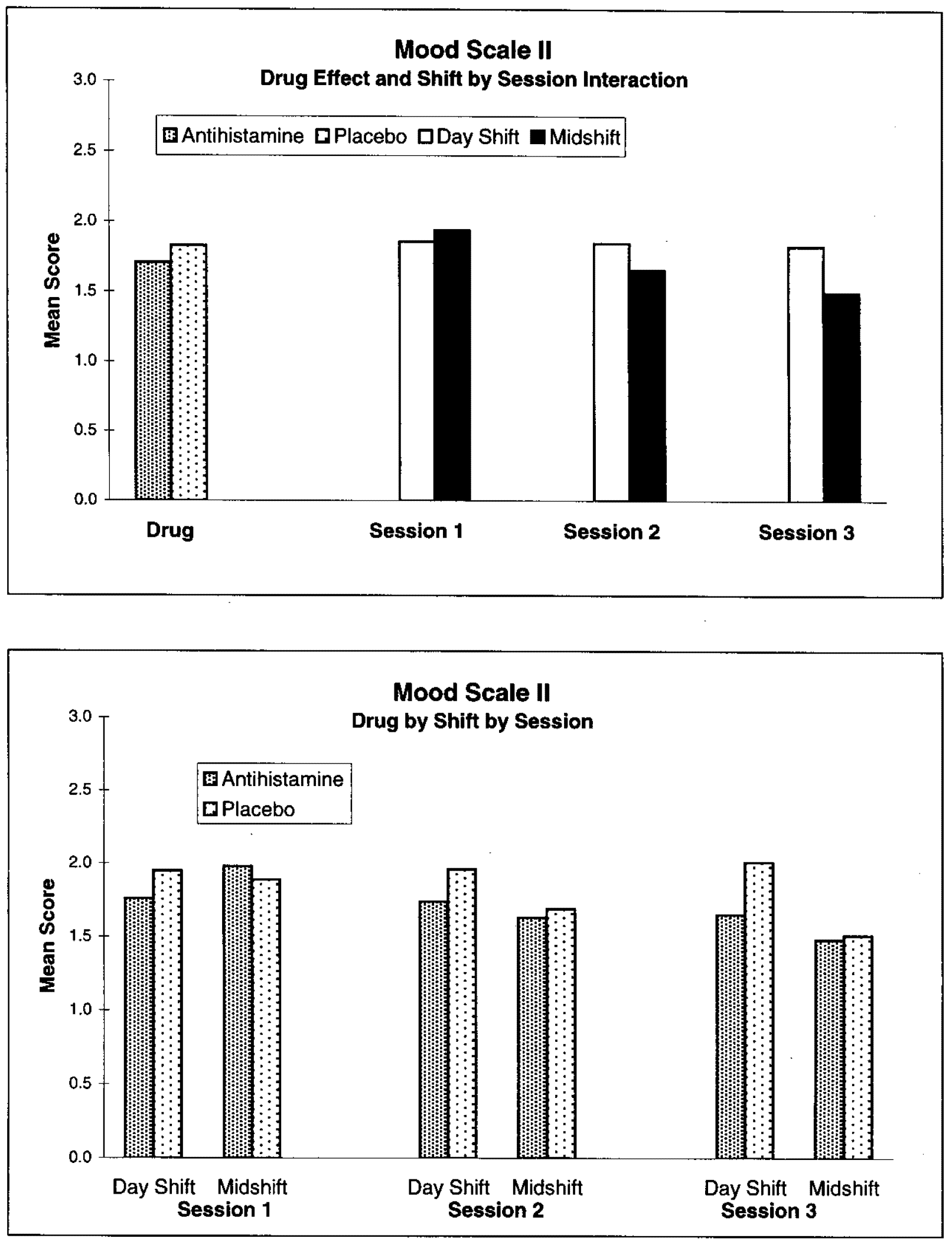

Figure 42. Mood Scale II Activity Scale. 

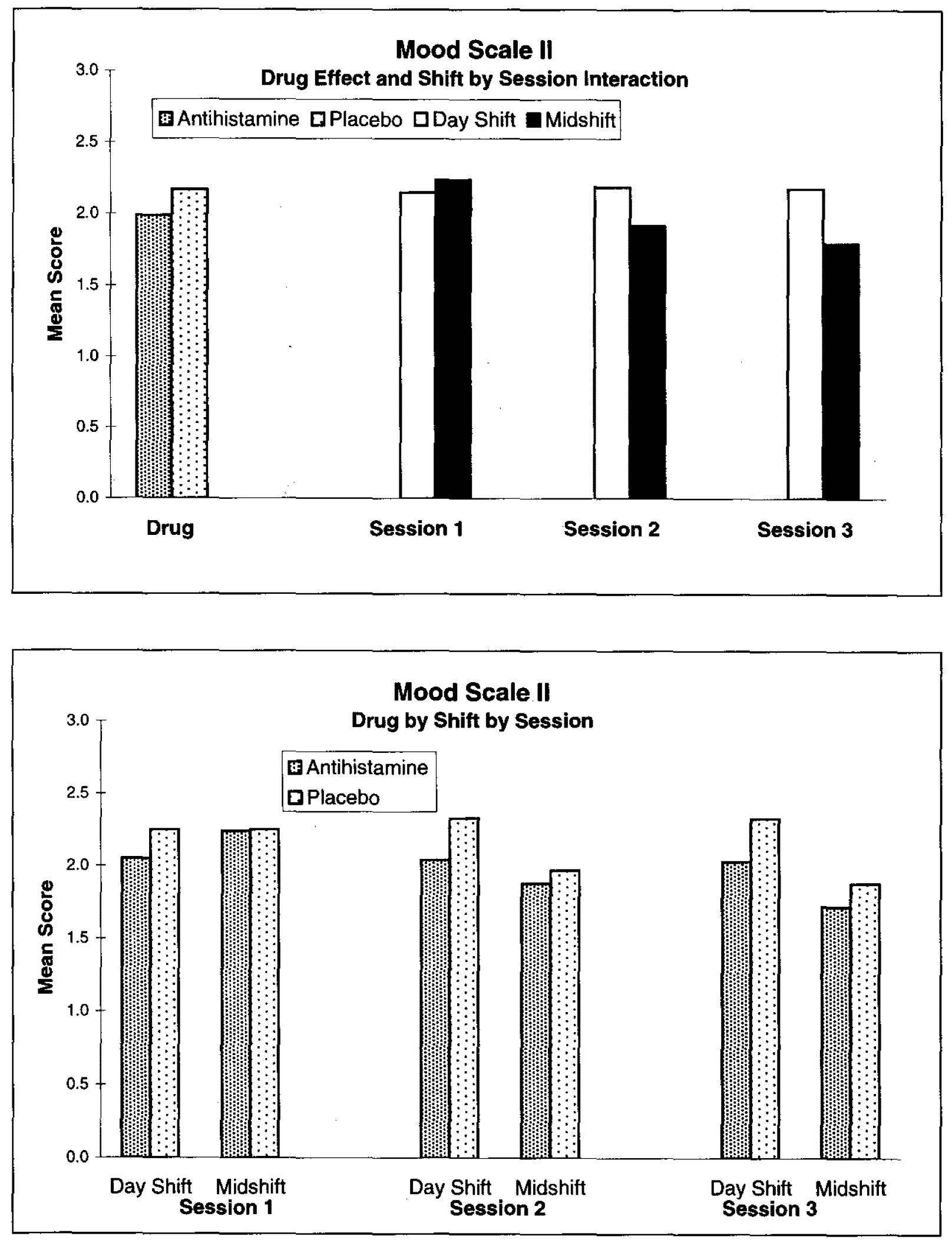

Figure 43. Mood Scale II Happiness Scale. 

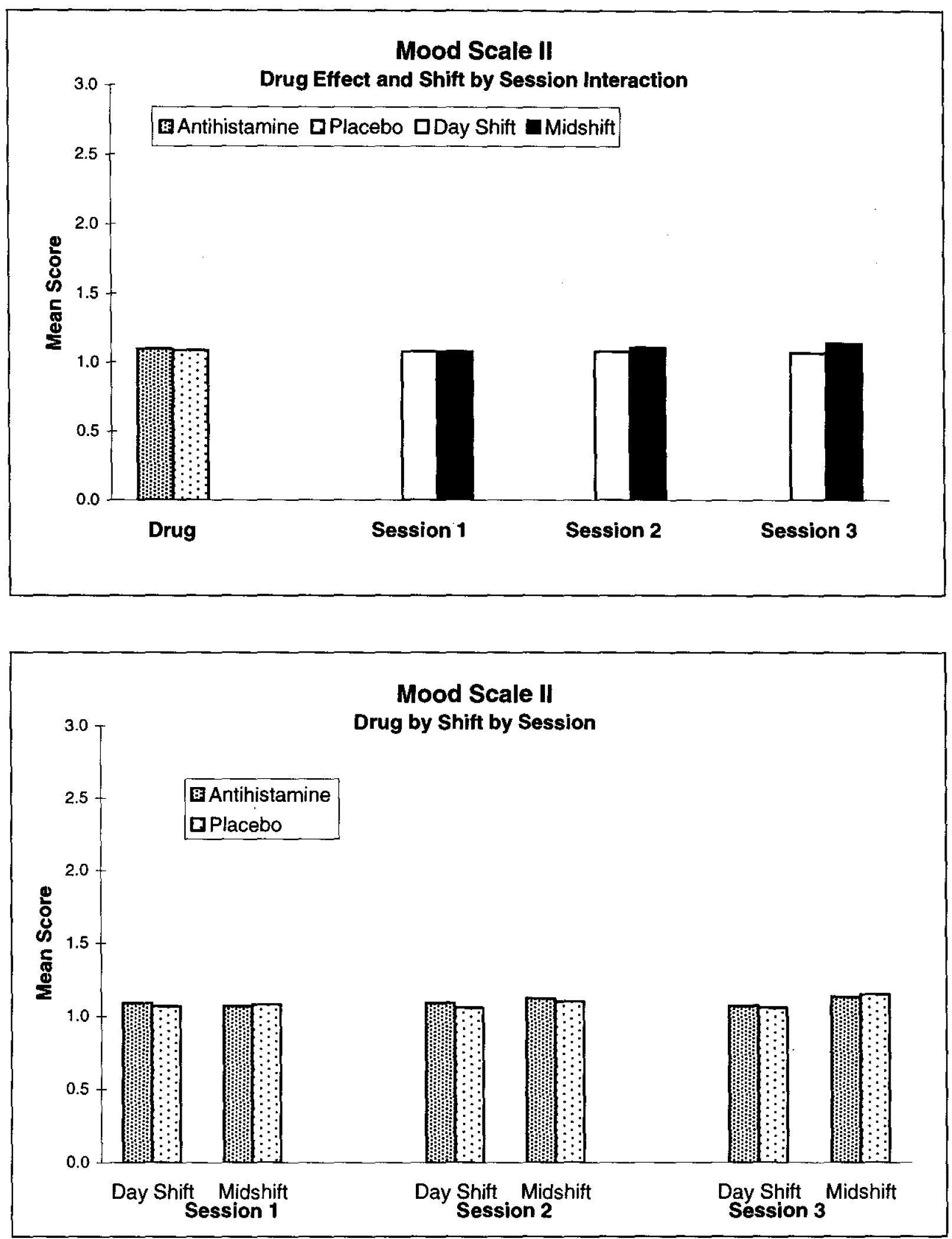

Figure 44. Mood Scale II Depression Scale. 

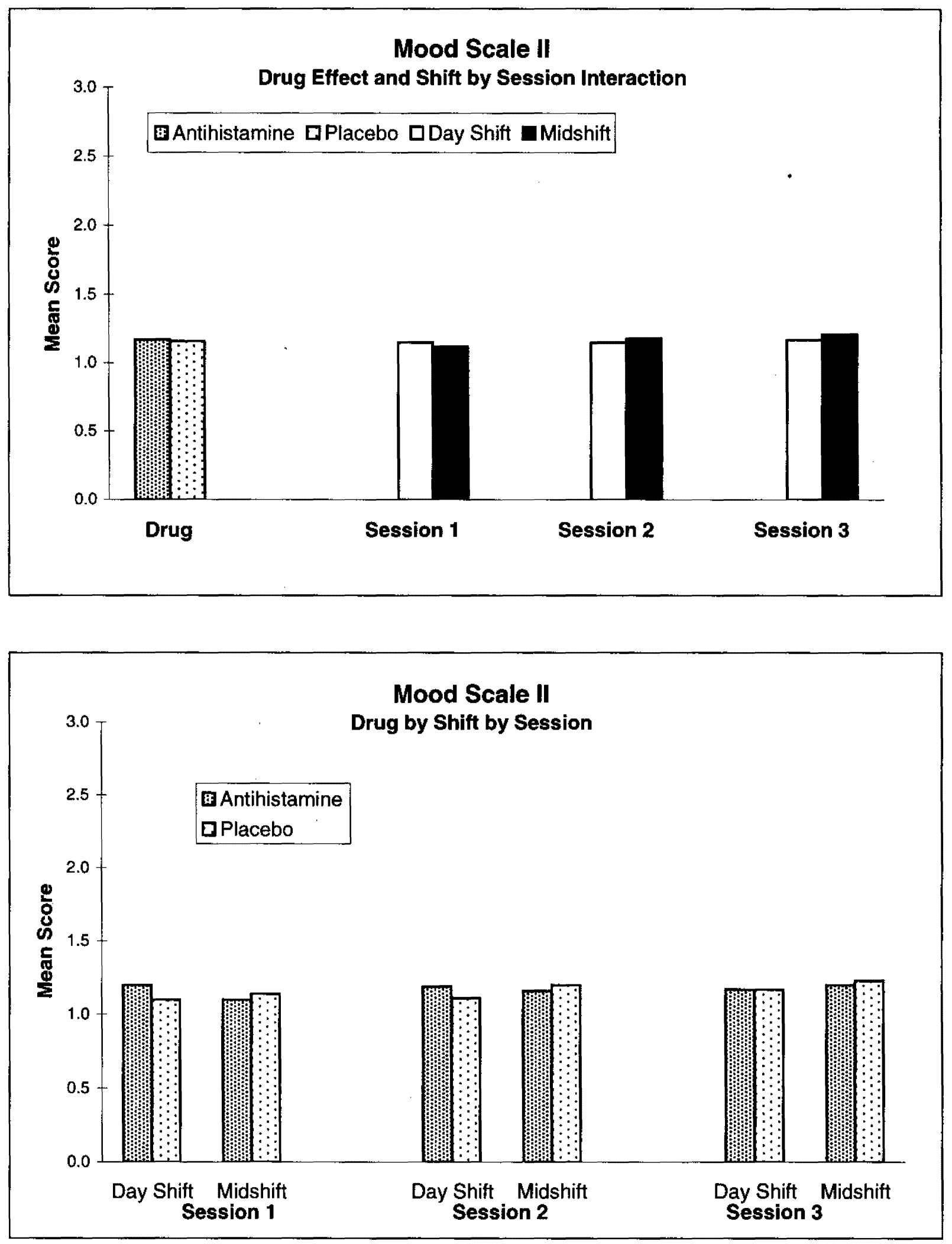

Figure 45. Mood Scale II Anger Scale. 

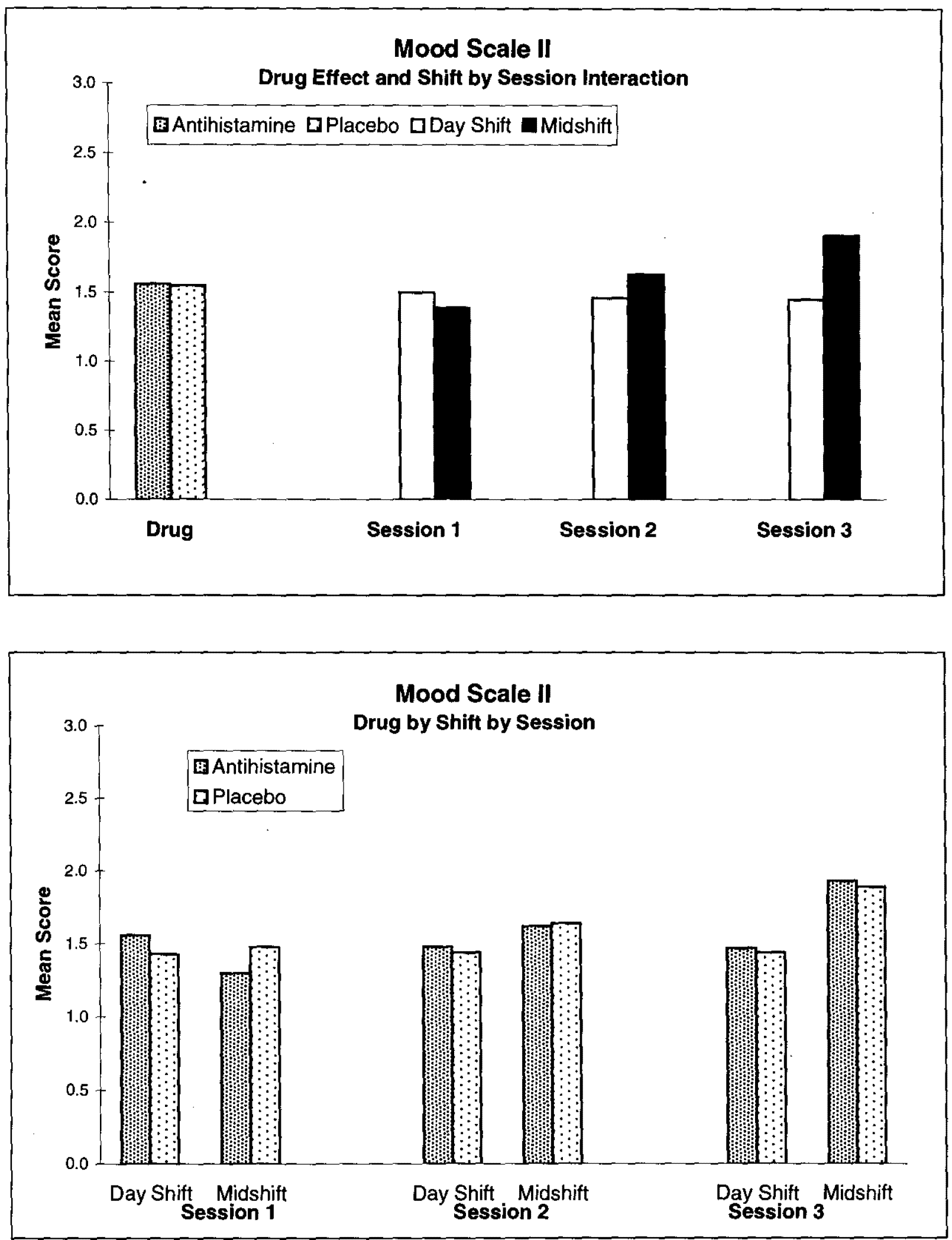

Figure 46. Mood Scale II Fatigue Scale. 

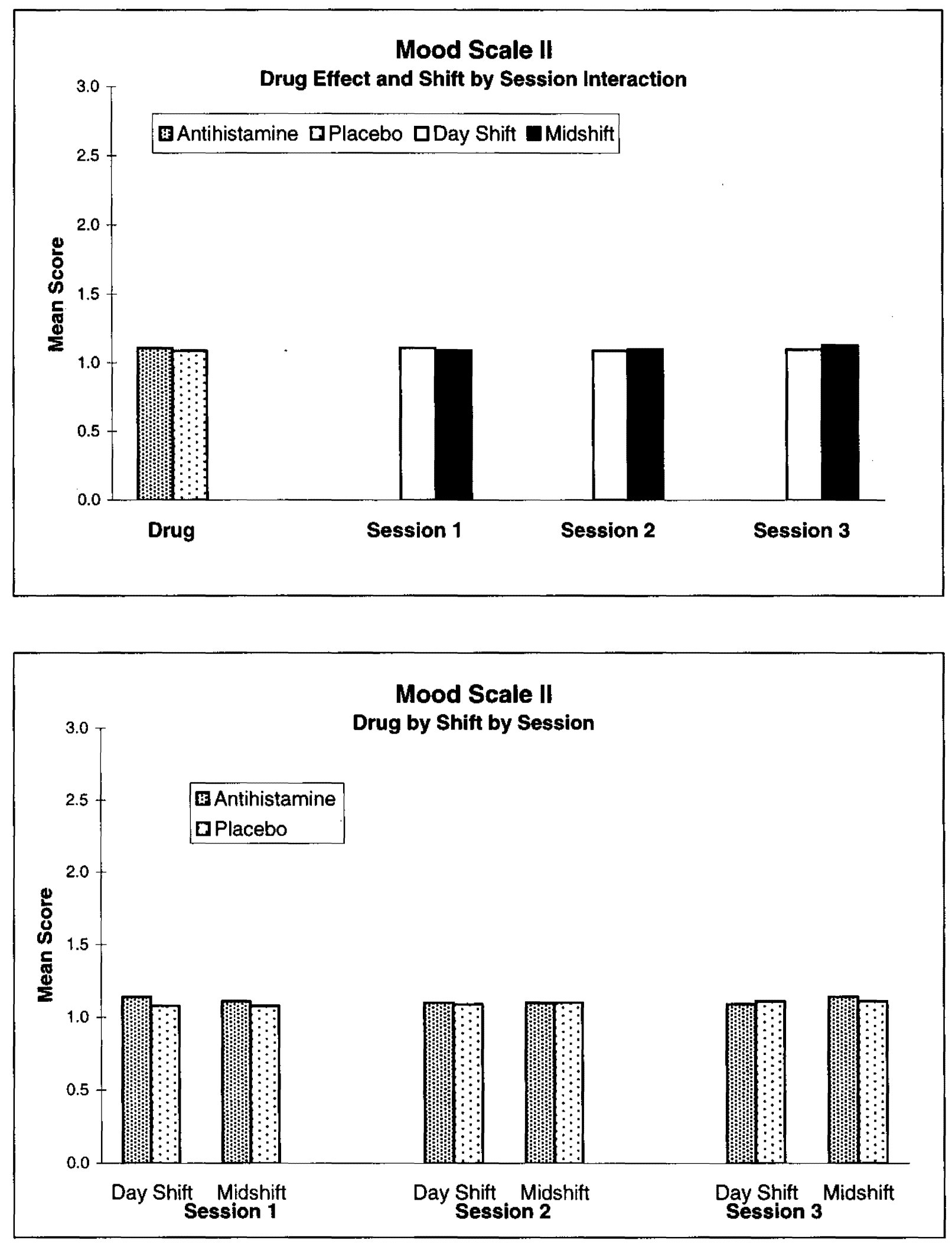

Figure 47. Mood Scale II Fear Scale. 

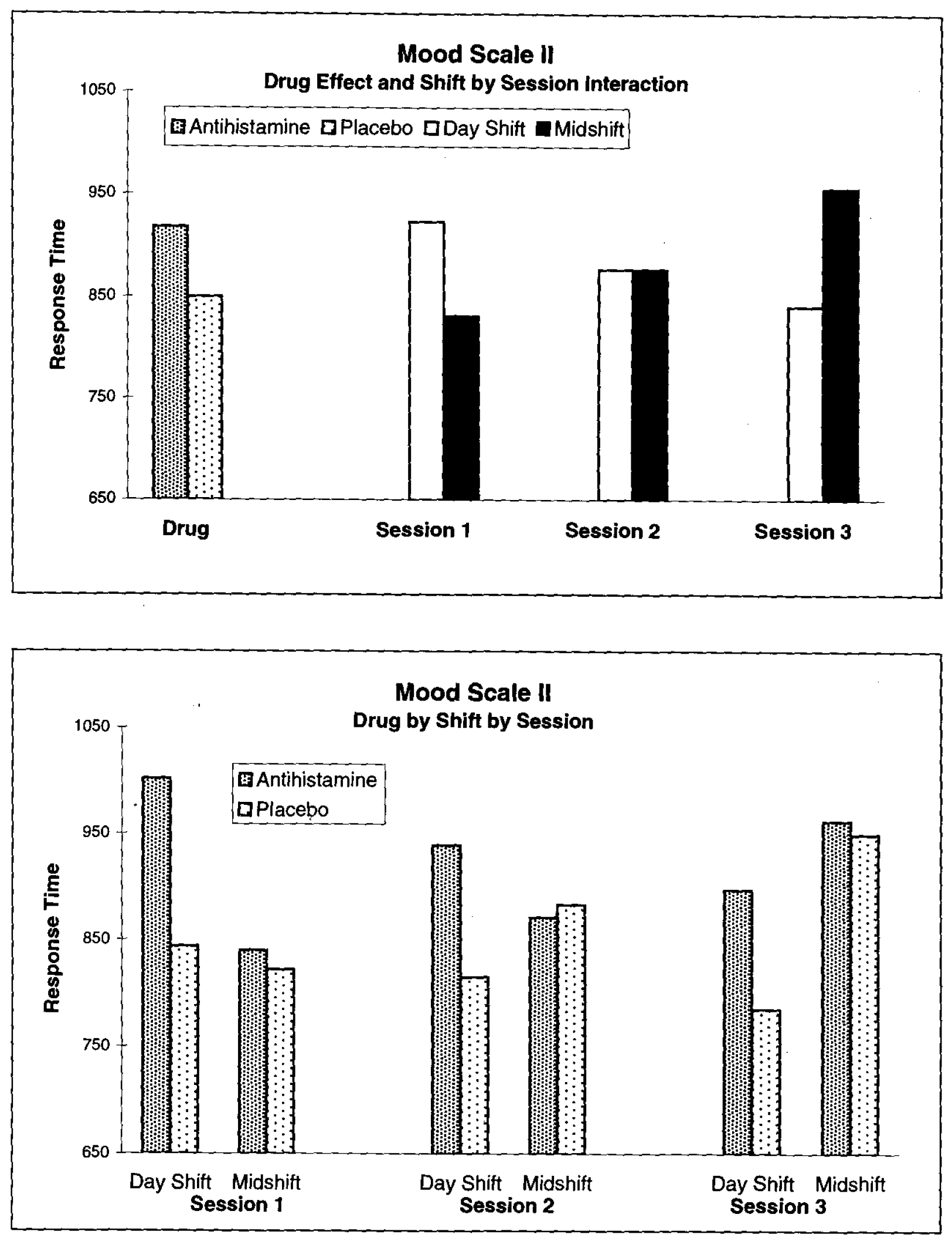

Figure 48. Mood Scale II Response Time. 
TABLES

Table 1. Summary of Task Codes.

\begin{tabular}{|rl|}
\hline \multicolumn{1}{|c|}{ Task } & Code \\
\hline Spatial Processing & SPA \\
Critical Tracking & TRK \\
Dual Task (Group Lambda) & DULG \\
Dual Task (Individual Lambda) & DULI \\
IML Attention Switching & NTI \\
NovaScan ${ }^{\text {TM FAA }}$ & NSF \\
Air Traffic Scenarios Test & ATST \\
Multi-Attribute Task Battery & MATB \\
Antihistamine Symptoms Questionnaire & AHSQ \\
Activity State Questionnaire & ACTSQ \\
Mood Scale II & MOOD \\
NASA Task Load Index & TLX \\
\hline
\end{tabular}


Table 2. Example Protocols for Dosing and Testing during Day Shift and Midshift Sessions.

\begin{tabular}{|c|c|c|}
\hline \multicolumn{3}{|c|}{ Day Shift } \\
\hline Time & Group 1 & Group 2 \\
\hline 800 & Dose 1 & \\
\hline 900 & \multirow[t]{2}{*}{ Session 1} & \\
\hline 1000 & & Dose 1 \\
\hline 1100 & Meal & \multirow[t]{2}{*}{ Session 1} \\
\hline 1200 & Dose 2 & \\
\hline 1300 & \multirow[t]{2}{*}{ Session 2} & Meal \\
\hline 1400 & & Dose 2 \\
\hline 1500 & Snack & \multirow[t]{2}{*}{ Session 2} \\
\hline 1600 & Dose 3 & \\
\hline 1700 & \multirow[t]{2}{*}{ Session 3} & Snack \\
\hline 1800 & & Dose 3 \\
\hline 1900 & End & \multirow[t]{2}{*}{ Session 3} \\
\hline 2000 & & \\
\hline 2100 & & End \\
\hline
\end{tabular}

\begin{tabular}{|c|c|c|}
\hline \multicolumn{3}{|c|}{ Midshift } \\
\hline Time & Group 1 & Group 2 \\
\hline 2000 & Dose 1 & \\
\hline 2100 & Session 1 & \\
\cline { 1 - 1 } 22200 & & Dose 1 \\
\hline 2300 & Meal & Session 1 \\
\cline { 1 - 1 } 2400 & Dose 2 & \\
\hline 100 & Session 2 & Meal \\
\cline { 1 - 1 } 200 & & Dose 2 \\
\hline 300 & Snack & Session 2 \\
\cline { 1 - 1 } 400 & Dose 3 & \\
\hline 500 & Session 3 & Snack \\
\cline { 1 - 1 } \cline { 1 - 1 } & & Dose 3 \\
\hline 700 & End & Session 3 \\
\hline 800 & & \\
\hline 900 & & End \\
\hline
\end{tabular}

Table 3. Individualized Lambda Values for Dual Task.

\begin{tabular}{|c|c||c|c|}
\hline ID & Lambda & ID & Lambda \\
\hline \hline 201 & 4.0 & 224 & 3.3 \\
204 & 3.6 & 225 & 3.7 \\
206 & 3.9 & 226 & 4.2 \\
211 & 3.9 & 227 & 3.4 \\
216 & 4.7 & 229 & 3.2 \\
217 & 3.9 & 230 & 3.6 \\
218 & 4.0 & 232 & 3.3 \\
223 & 3.4 & 233 & 3.7 \\
\hline
\end{tabular}




\section{Table 4. Summary of Significant ANOVA Effects.}

(In small italics: $0.05<p<0.10$; all others: $p=.05$ )

\begin{tabular}{|c|c|c|c|c|c|c|}
\hline TASK & Drug & Shift & Session & Drug x Shift & Drug $x$ Session & Shift x Session \\
\hline $\begin{array}{l}\text { Spatial } \\
\text { Processing }\end{array}$ & $\mathrm{PC}$ & & & MNCORRT & & $\begin{array}{l}\text { MNCORRT } \\
\text { PC }\end{array}$ \\
\hline $\begin{array}{l}\text { Critical } \\
\text { Tracking }\end{array}$ & MEANL & & & & & $\begin{array}{l}\text { MEANL } \\
\text { MAXL }\end{array}$ \\
\hline $\begin{array}{l}\text { Dual Task } \\
\text { (Group Lambda) }\end{array}$ & $\begin{array}{l}\text { RMS } \\
\text { CTLOSS } \\
\text { MNCORRT } \\
\text { PC } \\
\text { THRPUT } \\
\end{array}$ & & RMS & & RMS & $\begin{array}{l}\text { RMS } \\
\text { CTLOSS } \\
\text { MNCORRT } \\
\text { PC } \\
\text { THRPUT } \\
\end{array}$ \\
\hline $\begin{array}{l}\text { Dual Task } \\
\text { (Indiv. Lambda) }\end{array}$ & $\begin{array}{l}\text { RMS } \\
\text { CTLOSS } \\
\text { MNCORRT } \\
\text { THRPUT } \\
\end{array}$ & & RMS & $P C$ & & $\begin{array}{l}\text { RMS } \\
\text { CTLOSS } \\
\text { MNCORRT } \\
\text { THRPUT } \\
\end{array}$ \\
\hline $\begin{array}{ll}\text { IML } & \text { Manikin } \\
\text { Attention } & \\
\text { Switching } & \end{array}$ & & MANPCX & MANPC & & & $\begin{array}{l}\text { MANCORRT } \\
\text { MANCORTX } \\
\text { MANTP }\end{array}$ \\
\hline Task Math & $\begin{array}{l}\text { MTHCORRT } \\
\text { MTHCORTX } \\
\text { MTHTP } \\
\end{array}$ & $\begin{array}{l}\text { MTHCORRT } \\
\text { MTHCORTX } \\
\text { MTHTP } \\
\end{array}$ & & & & \\
\hline $\begin{array}{lr}\text { NovaScan }^{\mathrm{TM}} & \mathrm{Vec} \\
\text { FAA Test } & \text { Mem }\end{array}$ & VECPC & MEMPC & MEMPC & & & VECCRT \\
\hline $\begin{array}{l}\text { Air Traffic } \\
\text { Scenarios Test } \\
\text { (ATST) }\end{array}$ & $D E L A Y$ & & PCDEST & & & DELAY \\
\hline $\begin{array}{lr}\text { Multi- } & \text { Monitor } \\
\text { Attribute } & \text { Comm. } \\
\text { Task } & \\
\text { Battery } & \text { Track } \\
\text { MATB) } & \text { Tanks }\end{array}$ & $\begin{array}{l}\text { MONRT } \\
\text { COMCRT } \\
\text { COMER } \\
\text { TRKRMS } \\
\text { TNKMAD }\end{array}$ & COMCRT & COMER & & COMCRT & $\begin{array}{l}\text { MONRT } \\
\text { COMCRT } \\
\text { COMER } \\
\text { TRKRMS } \\
\text { TNKMAD }\end{array}$ \\
\hline
\end{tabular}

\begin{tabular}{|c|c|c|c|c|c|c|}
\hline QUESTIONNAIRE & Drug & Shift & Session & Drug $x$ Shift & Drug $x$ Session & Shift x Session \\
\hline $\begin{array}{l}\text { Antihistamine } \\
\text { Symptoms } \\
\text { Questionnaire }\end{array}$ & & & & & & \\
\hline $\begin{array}{l}\text { Activity State } \\
\text { Questionnaire }\end{array}$ & & $\begin{array}{l}\text { PHYSICAL } \\
\text { PREPARED }\end{array}$ & $\begin{array}{l}\text { PHYSICAL } \\
\text { PREPARED }\end{array}$ & PHYSICAL & & $\begin{array}{l}\text { PHYSICAL } \\
\text { PREPARED }\end{array}$ \\
\hline Mood Scale II & $\left\{\begin{array}{l}\text { ACTIVITY } \\
\text { HAPPINESS } \\
\text { Overall } R T\end{array}\right.$ & $\begin{array}{l}\text { ACTIVITY } \\
\text { HAPPINESS } \\
\text { DEPRESSION } \\
\text { FATIGUE }\end{array}$ & $\begin{array}{l}\text { ACTIVITY } \\
\text { HAPPINESS } \\
\text { FATIGUE }\end{array}$ & ACTIVITY & & $\begin{array}{l}\text { ACTIVITY } \\
\text { HAPPINESS } \\
\text { DEPRESSION } \\
\text { ANGER } \\
\text { FATIGUE } \\
\text { Overall RT } \\
\end{array}$ \\
\hline $\begin{array}{l}\text { NASA-TLX } \\
\text { (ATST) }\end{array}$ & & & & & FRUSTRATION & \\
\hline $\begin{array}{l}\text { NASA-TLX } \\
\text { (MATB) }\end{array}$ & MENTAL & & & & & $\begin{array}{l}\text { MENTAL } \\
\text { PHYSICAL } \\
\text { PERFORMANCE } \\
\text { FRUSTRATION }\end{array}$ \\
\hline
\end{tabular}




\section{APPENDIX A \\ SUBJECT CHARACTERISTICS}




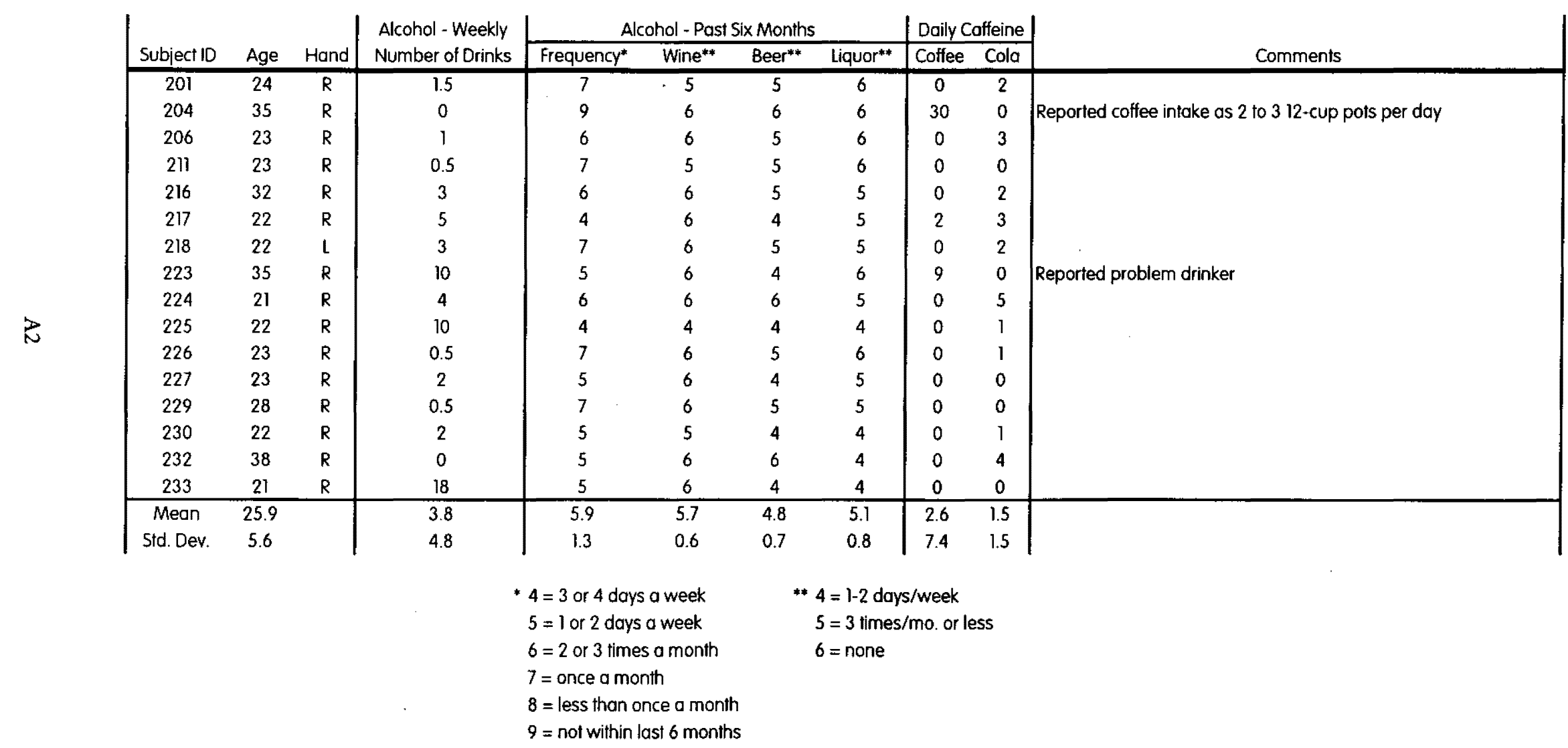


APPENDIX B

TASK DESCRIPTIONS 


\section{BRIEF DESCRIPTION OF TASKS}

Spatial Processing. This test, developed by Shingledecker (1984), uses four-bar histogram patterns. Each histogram bar can be one of six heights at random. At the beginning of each trial, the subject views a pattern. After a fixed delay, the pattern is immediately erased and a new one is presented. The subject must decide as quickly as possible if the new pattern is identical to the preceding pattern (except for a $90^{\circ}$ rotation). The subject then presses one key for "same" or another key for "different." As soon as the response is made, a new comparison pattern appears. Both mean reaction time for correct responses and percentage correct are used as dependent measures.

Tracking. This task, developed by McRuer and Jex (1967), requires that the subject maintain an unstable target in the center of a horizontal line by manipulating a control device to nullify the input disturbance. An instability parameter (lambda) is used to control the difficulty of the task. This parameter varies as a function of subject performance and actually serves as the primary performance measure.

Sternberg Memory Search. The general Sternberg paradigm (Sternberg, 1969) requires that subjects respond as rapidly and accurately as possible to visually presented letters. At the beginning of the test, a set of letters drawn randomly from a restricted alphabet is presented to the subject for memorization. The set of letters (positive set) stays on the screen for a maximum of five seconds, then the screen is cleared and a series of single test letters is presented. If the presented letter matches one of the letters in the previously memorized positive set, the subject responds "same" (key press). If a different letter appears (negative set), then the subject responds "different" (key press), indicating a non-matching letter was presented. The Sternberg task included in the Dual Task for this study uses a set size of four letters that are changed for each block of stimuli (session). Thus, a letter can be a target in one session and a distracter in another.

Dual Task - Tracking and Sternberg Memory Search. One of the most critical and potentially sensitive higher cognitive functions that might be susceptible to risk factor exposure is the ability of the subject to allocate attentional resources among several tasks. To investigate this, the present study used the time-sharing paradigm that has been well studied in cognitive psychology (Damos and Wickens, 1980; O’Donnell and Eggemeier, 1986; Damos, 1991). The specific form of this paradigm was the Dual Task included in the UTC-PAB (Perez, Masline, Ramsey, and Urban, 1987). This consists of the Sternberg task and the Tracking task being presented simultaneously.
In this implementation of the Dual Task, the Tracking task is presented in the middle of the screen and the letters of the Sternberg task appear in a fixed location directly above the center null point. The target of the compensatory tracking task moves laterally. A different four-letter memory set was used for each session. That is, only one positive memory set was presented with several probe letters for each daily session. For a recent study discussing the implementation of the Dual Task when investigating the effects of antihistamines on military weapon system controllers, see Nesthus, Schiflett, Eddy, and Whitmore (1991).

Attention Switching Task - Manikin and Mathematical Processing. Time-sharing, as explained above in the Dual Task, is different from attention switching, another required attentional process that could be sensitive to risk factors. Workers must often make rapid shifts in attentional focus, as well as in the skills required to respond to a change in task demands. This externally-directed behavior defies automaticity in any true sense, since it must be flexible enough to respond to unusual demands. Thus, a test is needed to probe the subject's ability to shift attention and resource allocation in response to rapidly changing and unpredictable external demands. Such a procedure has been created using two tasks currently in the UTC-PAB.

In this procedure, the subject has two distinct and discrete tasks to perform. One is a spatially-based task, and the other is a mathematically-based task. Each of these appears, side-by-side, simultaneously on every stimulus screen. However, an indicator appears at the same time directing the subject to the task that is "active" (i.e., requires a response). The subject must make an exclusive response to the active task, where reaction time and percent correct data are obtained only for that task. The switching from task to task for each stimulus is random (within constraints). Therefore, the subject must remember to watch the indicator, allocate the appropriate resources to respond to that stimulus, and then make the appropriate response. This paradigm provides a test of the switching skills described above. Findings and results using this test can be found in O'Donnell (1991) and Schlegel, Shehab, and Gilliland (1994).

The two tests selected to exercise this paradigm are the Manikin test and the Mathematical Processing test. The Manikin test has a long history of use (Benson and Gedye, 1963; Reader, Benel, and Rahe, 1981; Schlegel and Storm, 1983) and is presented in a wide variety of formats by military psychologists (Miller, Takamoto, Bartel, and Brown, 1985). As implemented in this experiment, a manikin "stick figure" is presented facing either forward or backward. 
In addition, the figure can be either upright or upsidedown. The figure is standing on a box and inside the box is either a rectangle or a circle. In the figure's two hands are a rectangle and a circle. The subject's task is to note which symbol is inside the box, and then to determine which of the manikin's hands is holding the designated symbol. The subject then presses the left or right of two keys corresponding to the manikin's left or right hand.

The Mathematical Processing test is based on similar tasks described by Perez et al. (1987). It presents three single-digit numbers that must be added or subtracted. If the answer is greater than 5, one response is given. If the answer is less than 5, another response is required. This task has been reported by Shingledecker (1984) to be a relatively pure index of mathematical functioning.

NovaScan $^{\text {TM }}$ FAA Task. This NovaScan ${ }^{\text {TM }}$ paradigm (NTI: O'Donnell, 1995) requires integrated responses to three tasks. For two of the tasks, stimulus screens are presented in directed attention fashion with a series of stimuli from one task alternating with a series of stimuli from the other task. In addition, a vigilance/attention task is performed for every stimulus screen. Thus, it is similar in form to the Attention Switching task, but only one task display is on the screen at a time. In the Visual Search and Vector Projection task, the subject searches for two labeled vectors, makes mental rotations of the vectors based on verbal on-screen instructions, and responds as to whether the rotated vectors would intersect either on or off the screen. In the Spatial Memory task, the subject memorizes the position and shape of a missing symbol for later comparison with the next spatial memory stimulus screen. For the Attention task, subjects look for the presence of small symbols in the corners of each screen. The test is based on a fixed number of stimuli and test time is thus a function of subject proficiency.

Air Traffic Scenarios Test. The ATST (Aerospace Sciences, 1991; Broach and Brecht-Clark, 1994; Gilliland and Schlegel, 1992; Weltin, Broach, Goldbach, and O'Donnell, 1992) provides an approximation of the air traffic control environment and involves the directing of planes to their destinations using altitude, speed, and heading changes. The work version of the task lasts 25 minutes and involves 40 to 45 airplanes.

Multi-Attribute Task Battery. The MATB (Comstock and Arnegard, 1992) is an approximation of the air crew operations environment and requires the simultaneous performance of a monitoring task (a set of lights and a set of dials), an auditory communications task, a compensatory tracking task, and a resource management task involving the monitoring and control of fuel tank levels. The work version of this task lasts 40 minutes.

Activity State Questionnaire. The Activity State Questionnaire is a version of the Pennebaker Physical Symptoms Checklist (Pennebaker, 1982) and consists of 25 items scored with a seven-point scale. It is used to assess the current state of physical health. Two additional items assessing level of preparedness for task performance were added.

Mood Scale II. The Mood Scale II is a variation of the Profile of Mood States (POMS; McNair, Lorr, and Droppleman, 1971). The Mood Scale II has 36 adjectives which address Activity, Happiness, Depression, Anger, Fatigue, and Fear.

NASA Task Load Index (TLX). The NASA TLX (Hart and Staveland, 1988) allows subjects to provide ratings of task workload using the categories of mental, physical, temporal, performance, effort, and frustration. This collection of ratings was obtained following each work sample task. The TLX is an integral part of the Multi-Attribute Task Battery but was programmed as a stand alone task to follow the Air Traffic Scenarios Test.

\section{REFERENCES}

Aerospace Sciences, Inc. (1991, November). Air Traffic Control Specialist pre-training screen preliminary validation: Final report. (DTFA-01-90-Y01034). Washington, DC: Federal Aviation Administration.

Benson, A.J., and Gedye, J.L. (1963). Logical processes in the resolution of orienting conflict. (RAF Rpt. 259). Farnborough, UK: Royal Air Force Institute of Aviation Medicine.

Broach, D., and Brecht-Clark, J. (1994). Validation of the Federal Aviation Administration Air Traffic Control Specialist pre-training screen. (DOT/FAA/ AM-94/4). Washington, DC: Federal Aviation Administration, Office of Aviation Medicine.

Comstock, J.R., and Arnegard, R.J. (1992). The Multi-Attribute Task Battery for human operator workload and strategic behavior research. (NASA-TM-104174).

Damos, D.L. (1991). Multiple task performance. London, UK: Taylor \& Francis, LTD.

Damos, D.L., and Wickens, C.D. (1980). The identification and transfer of timesharing skills. Acta Psychologica, 46, 15-39. 
Gilliland, K., and Schlegel, R.E. (1992). Evaluation of extended practice effects on the Air Traffic Scenarios Test. (DTFA-02-92-P-13359). Oklahoma City, OK: Federal Aviation Administration, Civil Aeromedical Institute.

Hart, S.G., and Staveland, L.E. (1988). Development of NASA-TLX (Task Load Index): Results of empirical and theoretical research. In P.A. Hancock and N. Meshkati (eds.) Human mental workload. New York: Elsevier Scientific Publishers.

McNair, D.M., Lorr, M., and Droppleman, L.F. (1971). Profile of mood states. San Diego, CA: Educational and Industrial Testing Service.

McRuer, D.T., and Jex, H.R. (1967). A review of quasi-linear pilot models. IEEE Transactions on Human Factors in Electronics, 8, 231-49.

Miller, J.C., Takamoto, G.M., Bartel, G.M., and Brown, M.D. (1985). Psychophysiological correlates of long-term attention to complex tasks. Behavior Research Methods, Instruments, and Computers, 17, 186-90.

Nesthus, T.E., Schiflett, S.G., Eddy, D.R., and Whitmore, J.N. (1991). Comparative effects of antihistamines on aircrew performance of simple and complex tasks under sustained operations. (ALTR-91-104). Brooks AFB, TX: USAF Armstrong Laboratory, Crew Technology Division. (NTIS \# AD A248 752/8).

O'Donnell, R.D. (1991). Scientific validation of the Novascan (tm) tests: Theoretical basis and initial validation studies. NTI Report to Nova Technology, Inc., 19460 Shenango Drive, Tarzana, CA: NTI, Incorporated.

O'Donnell, R.D. (NTI: 1995). The effect of alcohol and fatigue on an FAA readiness-to-perform test. (DOT/FAA/AM-95/24). Washington, DC: Federal Aviation Administration, Office of Aviation Medicine.
O'Donnell, R.D., and Eggemeier, F.T. (1986). Workload assessment methodology. In K.R. Boff et al. (eds.), Handbook of perception and human performance, Vol II, New York: Wiley, 42-1 to $42-49$.

Pennebaker, J.W. (1982). The psychology of physical symptoms. New York, NY: Springer-Verlag.

Perez, W.A., Masline, P.J., Ramsey, F.R., and Urban, K.E. (1987). Unified Tri-Services Cognitive Performance Assessment Battery: Review and methodology. (AAMRL-TR-87-007). Wright-Patterson AFB, OH: Armstrong Aerospace Medical Research Laboratory.

Reader, D.C., Benel, R.A., and Rahe, A.J. (1981). Evaluation of a manikin psychomotor task. (USAFSAM-TR-81-10). Brooks AFB, TX: USAF School of Aerospace Medicine.

Schlegel, R.E., and Storm, W.F. (1983). Speed-accuracy tradeoffs in spatial orientation information processing. Proceedings of the 27th Annual Meeting of the Human Factors Society, Santa Monica, CA: Human Factors Society.

Schlegel, R.E., Shehab, R.L., and Gilliland, K (1994). Microgravity effects on cognitive performance measures: Practice schedules to acquire and maintain performance stability. (AL-CF-TR-1994-0040). Brooks AFB, TX: USAF Armstrong Laboratory, Crew Technology Division.

Shingledecker, C.A. (1984). A task battery for applied human performance assessment research. (AFAMRLTR-84-071). Wright-Patterson AFB, OH: Air Force Aerospace Medical Research Laboratories.

Sternberg, S. (1969). The discovery of processing stages: Extensions of Donders' method. Acta Psychologica, 30, 276-315.

Weltin, M., Broach, D., Goldbach, K., and O'Donnell, R. (1992). Concurrent criterion-related validation of the Air Traffic Control Specialist pre-training screen. (DTFA-01-89-Y-01019). Washington, DC: Federal Aviation Administration. 


\section{APPENDIX C}

\section{PERFORMANCE MEASURES}




\section{REFERENCE GUIDE FOR TASK VARIABLES AND CODES}

\section{General Information}

The identification scheme for the antihistamine trials is the following four-character code:

$c_{1} c_{2} c_{3} c_{4}$

$c_{1} \quad$ h-antihistamine; $p$-placebo; $r$-refresher

$c_{2} \quad(1,2,3,4,5,6)$-refresher session number; d-daytime testing;

n-nightrime testing

$c_{3} \quad$ a-first group tested; $b$-second group tested; $x$-not used

$c_{4} \quad(1,2,3)$-test trial; $x$-not used

Examples: $\quad \mathrm{r} 5 \mathrm{xx}-\mathrm{fifth}$ refresher session

hdb3 - antihistamine, daytime, second test group, third test trial (dose)

\section{General Variables Used in Many or All Tasks}

ID Subject identification number; subjects for antihistamine study were: $201,204,206,211,216,217,218,223,224,225,226,227,229,230,232,233$

SESSION Session number; antihistamine study consisted of Sessions 51 through 68

DATE Date of session

TIME Time of session

TASK Task name

INST Whether or not instructions were included (indicated by $-\mathrm{N} 1$ for instructions)

LENGTH Program option to specify task length

\section{Antihistamine State Scale (ASH)}

TOTAL Total score for antihistamine symptom impact

\section{Mood Scale (MOOD)}

$\mathbf{x x x N}$ Total number of adjective responses in category $\mathrm{xxx}$

xxxSUM Sum of scores for adjectives in category $\mathrm{xxx}$

$\mathbf{x x x M N}$ Mean of scores for adjectives in category $\mathbf{x x x}$

xxxPCT Percent score for category xxx; (xxxCT = $[\mathbf{x x M N}-1] / 2)$

$\operatorname{xxxT}$ Average response time for responses to adjectives in category $x x x$

RTALL Overall response time for all responses

$\begin{array}{ll}\text { xox } & \text { CATEGORY } \\ \text { ACT } & \text { Activity } \\ \text { HAP } & \text { Happiness } \\ \text { DEP } & \text { Depression } \\ \text { ANG } & \text { Anger } \\ \text { FAT } & \text { Fatigue } \\ \text { FER } & \text { Fear }\end{array}$




\section{Activity State Questionnaire (ACTSQ)}

PHYSICAL Total (weighted) score for physical state

PREP Total (weighted) score for preparedness

\section{Spatial Processing (SPA)}

MNCORRT Mean correct response time

SDCORRT Standard deviation of correct tesponse times

$\mathrm{N} \quad$ Number of stimuli

PC Percent correct stimuli

PINC Percent incorrect stimuli

PLAPSE Percent lapsed (i.e., timed-out) stimuli

NC Number of correct stimuli

NINC Number of incorrect stimuli

NLAPSE Number of lapsed (i.e., timed-out) stimuli

MNCRTPOS Mean correct response time for positive stimuli

SDCRTPOS Standard deviation of correct response times for positive stimuli

NPOS Number of positive stimuli

PCPOS Pẹrcentage correct for positive stimuli

PINCPOS Percentage incorrect for positive stimuli

\section{Critical Tracking (TRK)}

MAXL Maximum lambda during trial

CTLOSS Number of control losses

RMS Average root mean square error

MEANL Mean of lambda's at control losses

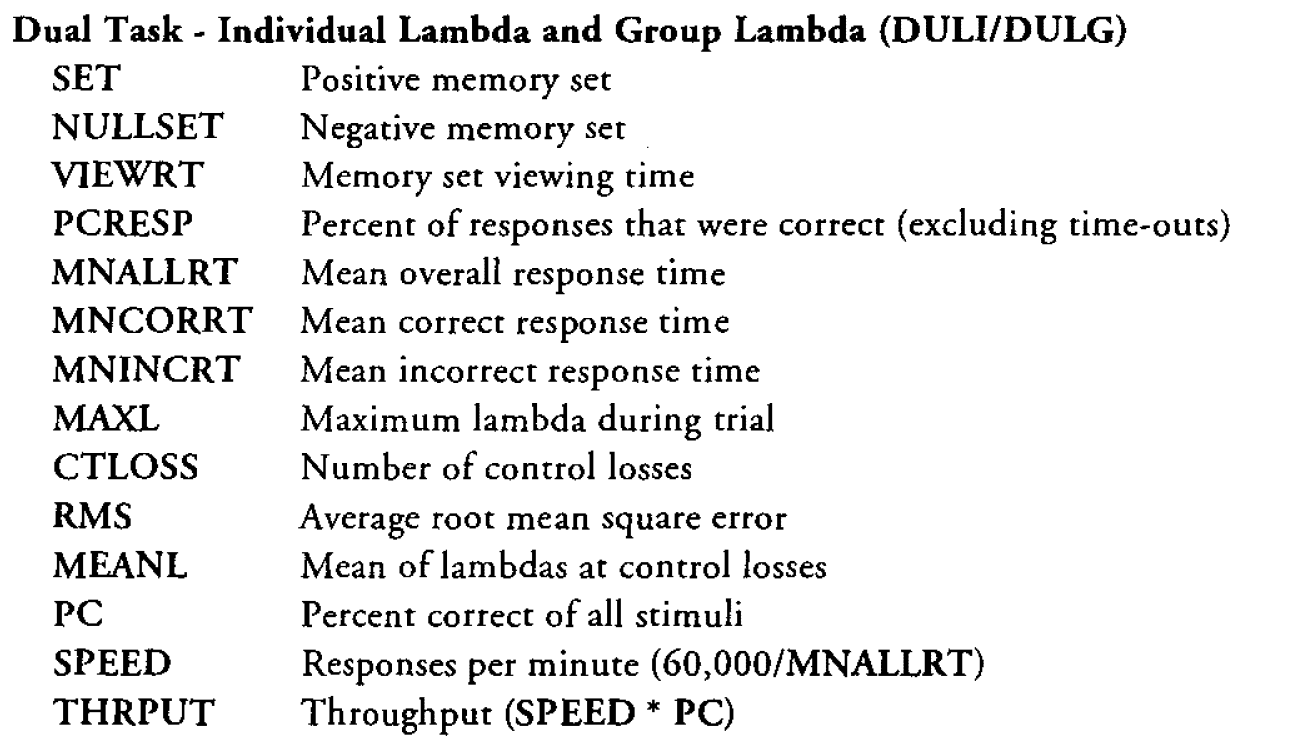



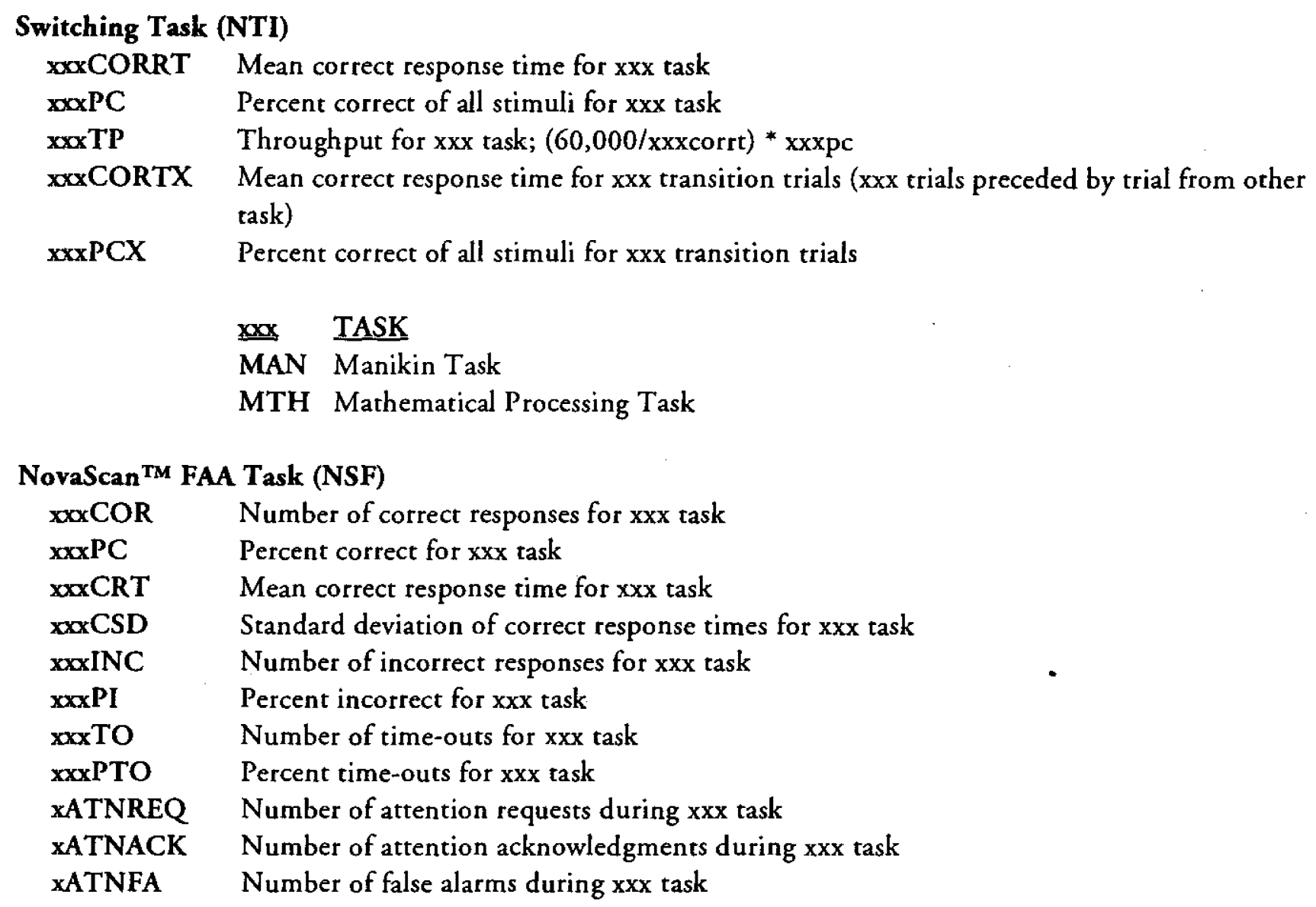

( $\underline{\mathbf{x}) \mathbf{x x}}$ TASK

(V)EC Visual Search and Vector Projection

(M)EMContinuous Spatial Memory

$\begin{array}{ll}\text { Air Traffic Scenarios Test (ATST) } \\ \text { SCEN } & \text { Scenario } \\ \text { CRSHAC } & \text { Number of crashes with other aircraft } \\ \text { CRSHBD } & \text { Number of crashes into air space boundary } \\ \text { CRSHAP } & \text { Number of crashes into the airport } \\ \text { SEPAC } & \text { Number of separation errors with other aircraft } \\ \text { SEPBD } & \text { Number of separation errors with air space boundary } \\ \text { ERRAPSPD } & \text { Number of speed errors at airport } \\ \text { ERRAPALT } & \text { Number of altitude errors at airport } \\ \text { ERRGTSPD } & \text { Number of speed errors at boundary gates } \\ \text { ERRGTALT } & \text { Number of altitude errors at boundary gates } \\ \text { ERRDEST } & \text { Number of destination errors } \\ \text { NDEST } & \text { Number of planes at destination } \\ \text { PCDEST } & \text { Percentage of planes at destination }\end{array}$


DELAY Delay score in routing planes for planes arriving at destination

NDIR Number of direction changes

NALT Number of altitude changes

NSPD Number of speed changes

TLX for ATST

MENTAL Rating of mental workload

PHYSICAL Rating of physical workload

TEMPORAL Rating of time-related workload

PERFORM Rating of performance

EFFORT Rating of required effort

FRUST Rating of frustration level

Multi-Attribute Task Battery (MATB)

SCRIPT Specific MATB run script

Systems Monitoring

LTSRT Mean response time for lights

DLSRT Mean response time for dials

MONRT Mean response time for lights and dials

LTSSD Standard deviation for lights

DLSSD Standard deviation for dials

MONSD Standard deviation for lights and dials

LTSTO Time Out errors for lights

DLSTO Time Out errors for dials

MONTO Time Out errors for lights and dials

LTSFA False Alarm errors for lights

DLSFA False Alarm errors for dials

MONFA False Alarm errors for lights and dials

LTSER Time Out and False Alarm errors for lights

DLSER Time Out and False Alarm errors for dials

MONER Time Out and False Alarm errors for lights and dials

MONKR Key Repeats (See explanation for COMRPT under Communications dependent variables below.)

Communications

COMCRT Mean response time for correct responses

COMCSD Standard deviation for correct responses

COMORT Mean overall response time

COMOSD Standard deviation for overall responses

COMER Total number of errors

(This includes othership false alarms, othership accuracy etrors, unexplained errors, ownship accuracy errors, and ownship time-outs. It does not include repeated ENTERs, described below.) 
COMYFA Othership false alarms

(correct radio and frequency, but message was for other ship)

COMYAC Othership accuracy errors

(Message was for other ship; either radio or frequency were incorrect.)

COMYIG Othership messages correctly ignored.

COMAC Accuracy errors

(response to ownship message, but either radio or frequency incorrect)

COMTO Time out errors

COMUNER Unexplained errors

(some response without identifiable cause, possibly false alarm)

COMRPT Repeated ENTERs (Number of times ENTER was pressed within 5 seconds of a previous ENTER press. Some subjects hold the ENTER key down for several seconds during this task. Matproc does not count these repeats as errors, but reports them with this dependent variable.)

Tracking

TRKRMS Root Mean Square (calculated for the each entire epoch)

Resource Management

TNKMAD Mean absolute deviation of tanks A and B from 2500

TNKAMN Mean of Tank A

TNKBMN Mean of Tank B

TNKACT Tank activity (number of pump changes ON or OFF)

Workload Rating Scale

TLXOMN Overall mean of subscales

TLXMEN Mean for Mental Demand subscale

TLXPHS Mean for Physical Demand subscale

TLXTMP Mean for Temporal Demand subscale

TLXPER Mean for Performance subscale

TLXEFT Mean for Effort subscale

TLXFRU Mean for Frustration subscale

TLXDUR Mean for duration of rating screen presentation 